\title{
Phenomenological analysis of Higgs boson production through gluon fusion in association with jets
}

\author{
Nicolas Greiner, ${ }^{a}$ Stefan Höche, ${ }^{b}$ Gionata Luisoni, ${ }^{c}$ Marek Schönherr, ${ }^{d}$ \\ Jan-Christopher Winter ${ }^{c}$ and Valery Yundin ${ }^{c}$ \\ ${ }^{a}$ DESY Theory Group, \\ Notkestr. 85, D-22607 Hamburg, Germany \\ ${ }^{b}$ SLAC National Accelerator Laboratory, \\ 2575 Sand Hill Rd, Menlo Park, CA 94025, U.S.A. \\ ${ }^{c}$ Max-Planck-Institut für Physik, \\ Föhringer Ring 6, D-80805 München, Germany \\ ${ }^{d}$ Physik-Institut, Universität Zürich, \\ Wintherturerstrasse 190, CH-8057 Zürich, Switzerland \\ E-mail: nicolas.greiner@desy.de, shoeche@slac.stanford.edu, \\ luisonig@mpp.mpg.de, marek.schoenherr@physik.uzh.ch, \\ jwinter@mpp.mpg.de, yundin@mpp.mpg.de
}

ABSTRACT: We present a detailed phenomenological analysis of the production of a Standard Model Higgs boson in association with up to three jets. We consider the gluon fusion channel using an effective theory in the large top-quark mass limit. Higgs boson production in gluon fusion constitutes an irreducible background to the vector boson fusion $(\mathrm{VBF})$ process; hence the precise knowledge of its characteristics is a prerequisite for any measurement in the VBF channel. The calculation is carried out at next-to-leading order (NLO) in QCD in a fully automated way by combining the two programs GoSAM and SHERPA. We present numerical results for a large variety of observables for both standard cuts and VBF selection cuts. We find that for all jet multiplicities the NLO corrections are sizeable. This is particularly true in the presence of kinematic selections enhancing the VBF topology, which are based on vetoing additional jet activity. In this case, precise predictions for the background can be made using our calculation by taking the difference between the inclusive $\mathrm{H}+2$ jets and the inclusive $\mathrm{H}+3$ jets result.

KEYwords: NLO Computations, Hadronic Colliders

ArXIV EPRINT: 1506.01016 


\section{Contents}

1 Introduction 1

2 Calculational setup 3

2.1 Virtual corrections 3

2.2 Real emission and phase space integration 3

2.3 Definitions relevant to the calculation 3

2.4 Ntuples generation and usage 4

2.5 Kinematic requirements and parameter settings 4

3 Higgs boson plus jets phenomenology $\quad 6$

$\begin{array}{ll}3.1 & \text { Cross sections, scale dependence and technicalities }\end{array}$

$\begin{array}{lll}3.2 & \text { Single-particle observables } & 17\end{array}$

3.3 Multi-particle observables and correlations 22

3.4 Multi-jet ratios at NLO 24

3.5 Comparing tagging jet selections and testing high-energy effects 30

4 Vector boson fusion phenomenology $\quad 34$

$\begin{array}{lll}4.1 & \text { Cross sections and scale dependence } & 35\end{array}$

$\begin{array}{lll}4.2 & \text { Differential observables } & 39\end{array}$

5 Conclusions $\quad 52$

\section{Introduction}

The discovery of a Higgs boson [1, 2] during Run I of the Large Hadron Collider (LHC) has ushered in an era of precision measurements to determine the nature of the new particle. A major step was the analysis of its spin structure [3-5], resulting in a very good agreement of the measurement with the Standard Model prediction. A second major step was the measurement of different production times decay rates by ATLAS [6-12] and CMS [13-16]. All experimental results point towards the mechanism of electroweak symmetry breaking [1720] being indeed as intimately linked to the generation of fermion masses as predicted by the Standard Model. This hypothesis will be further scrutinized during Run II of the LHC, where the Vector Boson Fusion (VBF) channel will play a leading role. In this production mode, a Higgs boson is created by annihilation of virtual $\mathrm{W}$ or $\mathrm{Z}$ bosons, radiated off the initial-state (anti-)quarks in a $t$-channel scattering process with no color exchange at leading order [21, 22]. The experimental signature thus consists of two forward jets, separated by a large rapidity gap with no hadronic activity [23-27]. This topology is the key to identifying 
the signal among an overwhelming number of backgrounds, which include Higgs boson production through gluon fusion accompanied by two or more jets. Despite the latter production mechanism of the Higgs boson being dominant, it can be largely suppressed compared to the VBF channel by requiring a large rapidity separation between the leading two jets (called the tagging jets), a large invariant mass of the corresponding dijet system, and an additional veto on jet activity in the central rapidity region. Higher-order QCD and EW corrections are known to alter the signal rate only insignificantly under these cuts [28-32]. In the present article, we investigate to which extent QCD corrections alter the background.

We also present a comprehensive analysis of $\mathrm{H}+3$ jets production in its own right. The high phenomenological relevance of the gluon fusion channel has spurred an unprecedented effort in the theoretical community. For Higgs boson production in conjunction with up to two jets, the NLO corrections have been available for some time [33-51]. In addition it has been shown how to include parton shower resummation on top of the fixed-order result. Especially, in the case of Higgs boson plus two jets, this has been demonstrated recently $[52,53]$. The first computation of Higgs boson production in association with three jets was accomplished just two years ago [54]. The development of the improved reduction algorithm NiNJA [55-57] to compute the virtual corrections then allowed a first phenomenological analysis that was published in ref. [58]. For the Higgs boson plus one jet final state, the full NNLO QCD results were computed lately [59-62] whereas the NNLO results for inclusive Higgs boson production have been around for a decade [6365]. More recently, the latter results have been combined with parton showers for the first time [66, 67], while the fixed-order frontier has been pushed further with a seminal calculation of the NNNLO corrections that has just been finalized [68-77].

In this article we focus on the behavior of the NLO results under different scale choices, and on the scaling with increasing number of jets. We also test for potential high-energy effects, which may require resummation [78, 79]. We note that the scaling of jet cross sections with increasing number of jets is comparable to $\mathrm{W}$ plus jets production [80, 81], once the number of jets is large enough. This can be understood using jet calculus [82, 83]. We also want to stress that the effect could be tested experimentally to a high accuracy by measuring the ratio of jet rate ratios between different processes. Systematic uncertainties should cancel to a large extent in this type of analysis.

In our calculations we use an effective gluon-to-Higgs boson coupling, where the top quark is treated as an infinitely heavy particle. Although this requires the top-quark mass to be much larger than the Higgs boson mass, the approximation has been shown to work very well [84-86]. Nonetheless, large corrections are to be expected when examining transverse momentum spectra.

This article is organized as follows: section 2 presents the technical prerequisites for our calculation. Section 3 discusses the properties of our results under generic cuts, which we also refer to as the baseline selection. In section 4 we finally focus on the experimentally most interesting case, which is the discussion of the VBF background predictions as well as their behavior under different selection rules for the tagging jets. Section 5 contains our conclusions and an outlook. 


\section{Calculational setup}

The calculation of the NLO corrections is performed by combining the two automated programs GoSAM $[87,88]$ for the generation and evaluation of the virtual one-loop amplitudes, and the Monte Carlo event generator Sherpa [89]. The two are linked using the Binoth Les Houches Accord [90, 91] — a standard for event and parameter passing between one-loop programs and Monte Carlo generators.

\subsection{Virtual corrections}

The GoSAm framework is based on an algebraic generation of $d$-dimensional integrands using a Feynman diagrammatic approach, employing QGraF [92] and Form [93, 94] for the diagram generation, and SPINNEY [95], HAGGIES [96] and Form to write an optimized Fortran output. For the reduction of the tensor integrals, we used NINJA [55-57], which is an automated package carrying out the integrand reduction via Laurent expansion. Alternatively, one can use other reduction techniques such as the standard OPP method [97-99] as implemented in SAMURAI [100] or methods of tensor integral reduction as implemented in Golem95 [101-103]. The resulting scalar integrals are evaluated using OnELoop [104].

We would like to stress that in comparison to the first computation [54], the integrand reduction performed by NINJA led to an improved timing as well as a better stability in the evaluation of the virtual amplitudes. This was a crucial aspect for both the analysis presented here and the one presented previously in [58].

\subsection{Real emission and phase space integration}

The calculation of the tree-level matrix elements, real emission contributions and subtraction terms in the Catani-Seymour framework [105], as well as the phase space integration have been performed by SHERPA [89]. This time we have solely used the matrix element generator Comix $[106,107]$ as opposed to the earlier calculations of refs. $[54,58]$ where we relied on a combination of MAdGraph $4[108,109]$, MADDipole $[110,111]$ and MADEVENT [112] for the calculation of real emission matrix elements, subtraction terms and the phase space integration of the real emission contribution. We recalculated the results obtained in $[54,58]$ with the SHERPA setup and found complete agreement. This is a very strong consistency check on both results.

\subsection{Definitions relevant to the calculation}

In the approximation of an infinitely heavy top quark, the Higgs boson coupling to gluons, which at LO is mediated by a top-quark loop, becomes independent of $m_{t}$, and can be described by an effective operator [113], as

$$
\mathcal{L}_{\text {eff }}=-\frac{c_{i}}{4} \mathrm{H} \operatorname{tr}\left(G_{\mu \nu} G^{\mu \nu}\right) .
$$

In the $\overline{\mathrm{MS}}$ scheme, the coefficient $c_{i}$ is given by $[33,34]$

$$
c_{i}=-\frac{\alpha_{\mathrm{s}}}{3 \pi v}\left(1+\frac{11}{4 \pi} \alpha_{\mathrm{s}}\right)+\mathcal{O}\left(\alpha_{\mathrm{s}}^{3}\right)
$$


in terms of the Higgs vacuum expectation value $v$, set to $v=246 \mathrm{GeV}$. The operator in eq. (2.1) leads to new Feynman rules, with vertices involving the Higgs boson field and up to four gluons.

In the absence of accompanying jets, i.e. for the pure process of Higgs boson production, it is natural to evaluate the strong coupling associated with the effective vertex at a scale equal to the Higgs boson mass $m_{\mathrm{H}}$. Once jets are present, it is more ambiguous to choose a natural scale. One possibility is to set up a mixed scale treatment where the two powers of the strong coupling in the effective vertex are frozen at $m_{\mathrm{H}}$, while the remaining powers of $\alpha_{\mathrm{s}}$ are evaluated at a different scale $\mu_{R}$. To adopt this strategy at NLO, an additional finite correction has to be added to the virtual contribution, taking account of the fact that the strong couplings in the Born contribution are computed at different scales [114].

In the case of Higgs boson production associated with $N$ jets, the general formula for computing the cross section is

$$
\frac{\mathrm{d} \sigma}{\mathrm{d} \Phi}=\alpha_{\mathrm{s}}^{N}\left(\mu_{R}\right) \alpha_{\mathrm{s}}^{2}\left(m_{\mathrm{H}}\right) B+\alpha_{\mathrm{s}}^{N+1}\left(\mu_{R}\right) \alpha_{\mathrm{s}}^{2}\left(m_{\mathrm{H}}\right)\left[V\left(\mu_{R}\right)+2 \beta_{0} \log \left(\frac{m_{\mathrm{H}}^{2}}{\mu_{R}^{2}}\right) B+R\right]
$$

where $B, V$ and $R$ denote the Born, the virtual and the real contribution, respectively, and $\beta_{0}$ is the one-loop beta function coefficient:

$$
\beta_{0}=\frac{11 C_{\mathrm{A}}-2 N_{\mathrm{F}}}{12 \pi} .
$$

In section 2.5 we will compare different settings for the renormalization and factorization scale and discuss their impact on the theoretical predictions.

\subsection{Ntuples generation and usage}

In order to simplify our analysis, the numerical results have been produced and stored in the form of Rоот Ntuples. This format is particularly useful if the definition of cuts and/or observables may change. The predictions incorporating such changes can be directly extracted from the given set of Ntuples without the need for generating new results. It also simplifies to vary the PDFs and scale choices, as this can be achieved without the reevaluation of the matrix elements, which is the most time consuming part of the calculation. The writing of the Ntuples is implemented in SHERPA, and we refer to ref. [115] for more details. Table 1 gives an overview of the different types and total number of Ntuple files which were generated for the analyses presented in the following sections. The files are available upon request.

\subsection{Kinematic requirements and parameter settings}

The numerical results presented in this study have been calculated for LHC center-of-mass energies of 8 and $13 \mathrm{TeV}$. We first discuss a set of basic cuts, which we use to define the baseline of our $\mathrm{H}+n$ jets analysis. In a second step, this selection will be refined by the application of a specific set of cuts favoring VBF topologies. In both cases jets are clustered using the anti- $k_{T}$ algorithm $[116,117]$ as implemented in the FASTJET package [118]. If not 


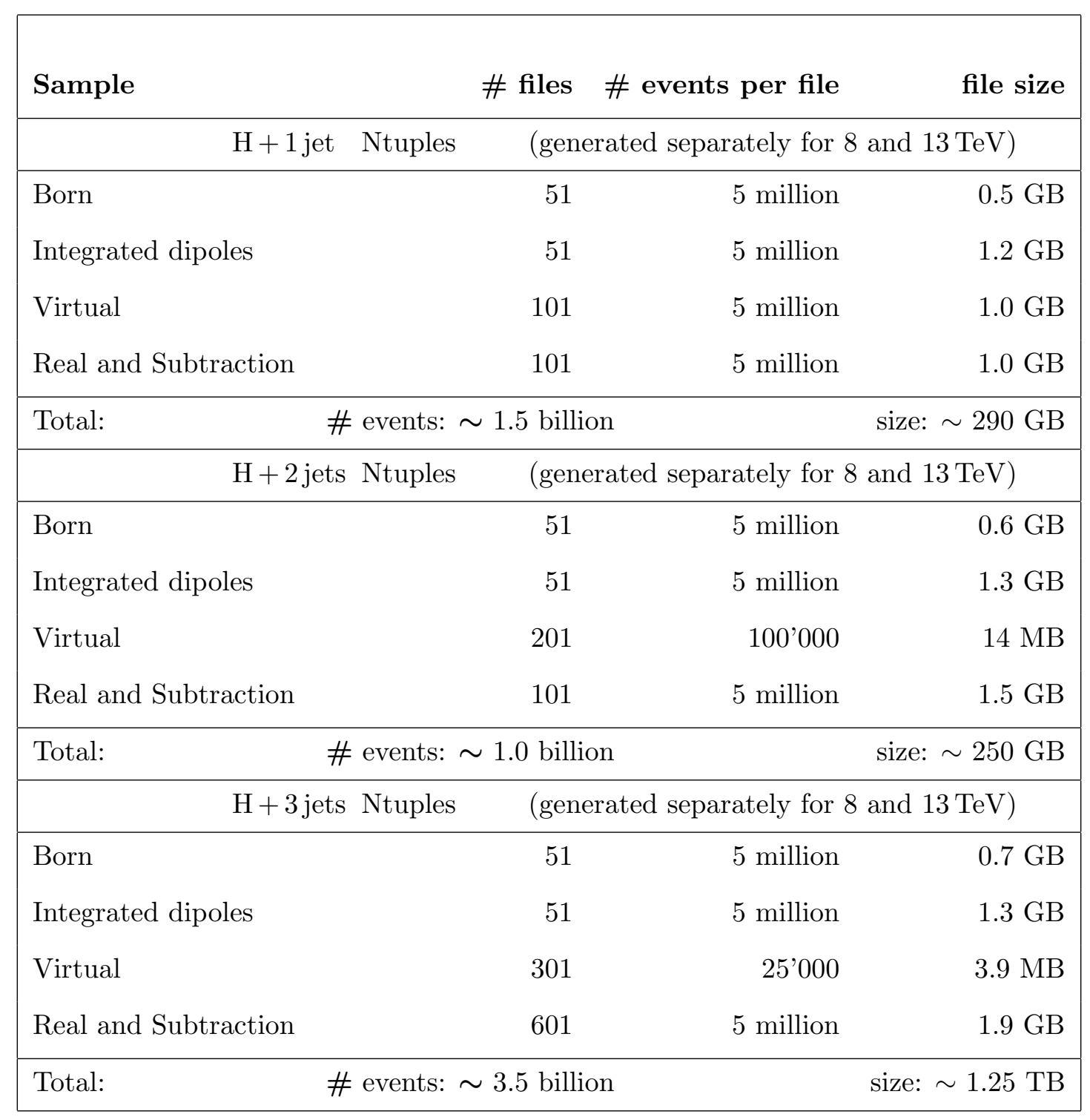

Table 1. Overview of the generated Ntuple files per jet bin and for the different types of events. As specified, each Ntuple sample is built from a number of (sub)files of certain size and contains a fixed number of events.

specified explicitly, the jet radius and PDF set have been set to $R=0.4$ and CT10nlo [119], respectively. The baseline selection is characterized by the following set of jet cuts:

$$
p_{T}>30 \mathrm{GeV}, \quad|\eta|<4.4 .
$$

For the VBF analyses, additional requirements on the kinematic properties of the tagging jets are imposed. They read

$$
m_{j_{1} j_{2}}>400 \mathrm{GeV}, \quad\left|\Delta y_{j_{1}, j_{2}}\right|>2.8 .
$$

If not stated otherwise these tagging jets, $j_{1}$ and $j_{2}$, are defined by the $p_{T}$ ordering of all jets in the event, i.e. the tagging jets are those with the highest and second highest transverse 
momentum. Different schemes to identify the tagging jets are also discussed and we refer to sections 3.5 and 4 where they are presented in comparison to the $p_{T}$ jet-tagging.

Concerning the choice of the renormalization and factorization scale, we define our central scale to be given by

$$
\mu_{F}=\mu_{R} \equiv \frac{\hat{H}_{T}^{\prime}}{2}=\frac{1}{2}\left(\sqrt{m_{\mathrm{H}}^{2}+p_{T, \mathrm{H}}^{2}}+\sum_{i}\left|p_{T, i}\right|\right)
$$

where the sum runs over all partons accompanying the Higgs boson in the event. This was shown to be a very reasonable scale choice in other multi-leg calculations [120]. However, it is not obvious to adopt this dynamical scale setting in the evaluation of all strong couplings occurring in the Higgs boson production processes. Because the top quark has been integrated out in the effective approach, one may argue that the strong coupling associated with the Higgs-gluon vertex should be fixed to its natural scale, which is of the order of $m_{\mathrm{H}}$. Furthermore, it is not obvious whether one should vary this scale together with the scales for the other powers of $\alpha_{\mathrm{s}}$ when performing the usual scale variation to assess the theoretical uncertainties. We note that there is no 'correct' choice and that the differences between the choices are formally of higher order. It is therefore interesting to investigate the effect of different scale setting approaches on the phenomenological results.

For our study, we consider three different scale choices, defined as

$$
\begin{array}{ll}
\text { A : } & \alpha_{\mathrm{S}}\left(x \cdot \frac{\hat{H}_{T}^{\prime}}{2}\right)^{3} \alpha_{\mathrm{s}}\left(x \cdot m_{\mathrm{H}}\right)^{2}, \\
\mathrm{~B}: & \alpha_{\mathrm{S}}\left(x \cdot \frac{\hat{H}_{T}^{\prime}}{2}\right)^{5}, \\
\mathrm{C}: & \alpha_{\mathrm{S}}\left(x \cdot m_{\mathrm{H}}\right)^{5} .
\end{array}
$$

The presence of the factor $x$ indicates that this scale is varied in the range $x \in[0.5,2.0]$. We note that we do not consider the older variant of scale $\mathrm{A}$, which was used by the previous computations of $\mathrm{H}+3$ jets $[54,58]$. In contrast to the scale $\mathrm{A}$ definition given here, the second term was always evaluated at $m_{\mathrm{H}}$ independent of the value of $x$. Consequently, a somewhat artificial reduction of the scale uncertainty was found, which we do not want to advocate here. We therefore omit this choice in our study.

\section{$3 \quad$ Higgs boson plus jets phenomenology}

In this section we discuss the results that have been obtained with the set of basic gluon fusion cuts as given in eq. (2.5).

\subsection{Cross sections, scale dependence and technicalities}

We start our analysis by presenting the results for the inclusive cross sections of the $\mathrm{H}+$ $n$ jets processes using the baseline kinematical requirements and the different scale choices discussed above. Figure 1 shows the total cross sections for both LO and NLO accuracy for the processes $\mathrm{H}+1$ jet, $\mathrm{H}+2$ jets and $\mathrm{H}+3$ jets at $E_{\mathrm{cm}}=8 \mathrm{TeV}$ (left plot) and $E_{\mathrm{cm}}=13 \mathrm{TeV}$ 

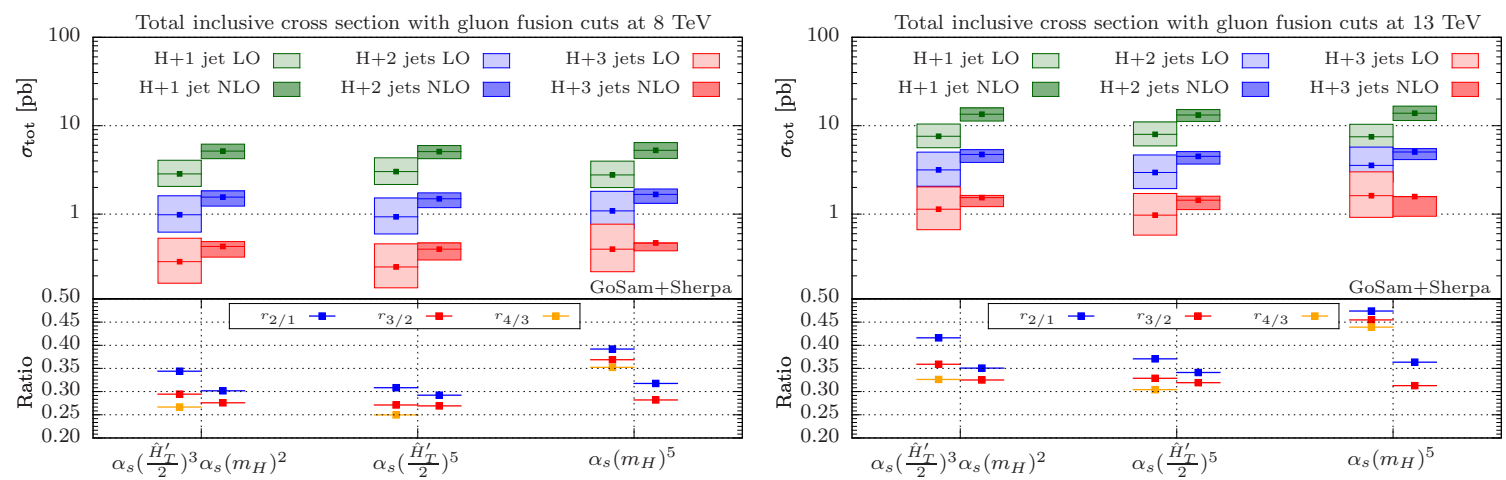

Figure 1. Total cross sections at LO (left side of each column) and NLO (right side of each column) for $\mathrm{H}+1$ jet (green), $\mathrm{H}+2$ jets (blue) and $\mathrm{H}+3$ jets (red) production at the LHC using different scale choices. In the lower part of the plots, the ratios $r_{2 / 1}$ (blue), $r_{3 / 2}$ (red) and $r_{4 / 3}$ (orange) are shown. Results have been obtained for center-of-mass energies of $8 \mathrm{TeV}$ and $13 \mathrm{TeV}$, and are visualized separately in the left and right panel, respectively.

(right plot). The cross sections are calculated for the three different scale choices A, B and $\mathrm{C}$ employing the definitions in eqs. (2.8). The upper part of each plot displays the LO and NLO results for the $\mathrm{H}+n$ jets processes where we use the size of the cross section boxes to visualize the effect of standard scale variations around the central scales for each of our three choices. The lower panels show the ratios

$$
r_{n / n-1}=\frac{\sigma_{\text {tot }}(\mathrm{H}+n \text { jets })}{\sigma_{\text {tot }}(\mathrm{H}+(n-1) \text { jets })}
$$

for $n=2,3,4$ at $\mathrm{LO}$ and $n=2,3$ at NLO accuracy.

Independent of the scale choice and order in perturbation theory, we see that the ratio $r_{2 / 1}$ is larger than the ratio $r_{3 / 2}$. One might expect this behavior for two reasons: on the one hand the $\mathrm{H}+2$ jets process features new kinematical topologies that do not occur in the $\mathrm{H}+1$ jet case. In particular in a $\mathrm{H}+2$ jets final state, configurations may arise where the Higgs boson is (almost) at rest, while in a $\mathrm{H}+1$ jet final state, the Higgs boson has to carry (at least) some finite transverse momentum, apart from the few jet-balanced real emission contributions. In other words, this example nicely demonstrates the increase in phase space that one obtains for the $\mathrm{H}+2$ jets case. On the other hand, one finds an effect because there are new partonic channels that open up for the $\mathrm{H}+2$ jets process, but do not exist in the $\mathrm{H}+1$ jet case. We note that the same reasoning cannot be applied explaining the transition from a second to a third jet. This transition neither opens additional phase space nor further partonic channels.

Comparing our dynamic scale choices, $\mathrm{A}$ and $\mathrm{B}$, we observe only mild differences in the predicted cross section ratios. We also find that the corresponding LO predictions are very similar to those obtained at the NLO. In particular for scale B, this agreement is found to be very good. Scale C seems to work well for the NLO results but yields increased ratios for the LO results. This is an indication that the fixed scale setup produces undesired effects one wants to avoid in an optimal scale choice. Raising the collider energy, we find the jet 
rate ratios to be enhanced but the overall pattern to be largely unchanged. This behavior is plausible as the effect is mainly driven by the increase in phase space for jet production.

One interesting point in this context is the fact that for scale $\mathrm{B}$, the ratio $r_{3 / 2}$ is very similar between LO and NLO. In addition, we have found great evidence, particularly from the $\mathrm{W} / \mathrm{Z}+$ jets calculations, that dynamical scales are more suitable for multi-leg processes. We therefore choose scale $\mathrm{B}$ as defined in eq. $(2.8 \mathrm{~b})$ as our default. It is also striking that the NLO ratios are almost identical for the two cases of $r_{2 / 1}$ and $r_{3 / 2}$. This hints at the possibility to extrapolate to the $r_{4 / 3}$ case. The idea has been worked out in ref. [81] for the case of W production in association with jets, and it is supported by jet calculus [82, 83]. However, in our case the fit to the function $r_{n / n-1}$ that was performed in ref. [81] would be trivial, with the first nontrivial check of staircase scaling being the ratio $r_{4 / 3}$ itself. In addition, figure 1 suggests that the quality of the fit will depend on the scale choice. Furthermore, it remains to be seen whether the good agreement for the ratios will hold for differential distributions.

Nevertheless a very interesting experimental opportunity opens up at this point: let us assume a set of hard processes that contain all possible kinematical topologies, and also all possible partonic channels, like $\mathrm{W} / \mathrm{Z}+2$ jets production and $\mathrm{H}+2$ jets production. Then it should be possible to test the universality of staircase scaling to a very good precision by measuring the ratio of jet rate ratios $r_{n / n-1}$ for different hard processes, such as $\mathrm{W} / \mathrm{Z}$ and $\mathrm{H}$ production. The differences between the dominant partonic production mechanisms at leading, next-to-leading and next-to-next-to-leading order will be reduced at higher jet multiplicity, and the ratio should be largely independent of $n$. A major deviation from this behavior would very likely signal the presence of new physics.

Having argued for our default scale choice, we want to give a more detailed summary of the total cross sections and their ratios based on using scale B. The results are presented in table 2, which lists the total cross sections for the one-jet, two-jet and three-jet process as well as their theoretical uncertainties from scale variations. It furthermore displays the global $K$-factors, the cross section ratios $r_{n / n-1}$ and the extra-jet fractions of the $\mathrm{H}+n$ jets samples denoted by $f_{n+1}$ where

$$
f_{n+1}=\frac{\sigma_{\geq n+1}(\mathrm{H}+n \text { jets })}{\sigma_{\text {tot }}(\mathrm{H}+n \text { jets })} .
$$

Jet radius dependence and PDF variation. Beyond the study of scale variation effects, it is interesting to investigate the variation and stability of the NLO results as a function of the jet radius, $R$. Focusing on the $\mathrm{H}+3$ jets case, the corresponding results are shown in the left and right panel of figure 2 for $8 \mathrm{TeV}$ and $13 \mathrm{TeV} \mathrm{LHC}$ center-of-mass energies, respectively. The cross sections are normalized to $\sigma_{\text {tot }}(R=0.4)$. At LO, they decrease with increasing $R$, because the probability for two partons to be clustered into a single jet increases, leading to a rejection of the event. From this basic consideration, it is clear that the dependence on the jet radius is stronger for $\mathrm{H}+3$ jets than it is for $\mathrm{H}+$ 2 jets, simply because an increasing number of jets necessarily leads to a reduction of the average distance in $R$-space between two jets.

At NLO the situation is more involved. We have to deal with a greater variety of parton configurations that will be classified as an inclusive (three-)jet event, while at LO 


\begin{tabular}{|c|c|c|c|c|c|c|c|c|}
\hline \multirow[t]{2}{*}{ Sample } & \multirow[b]{2}{*}{$\geq 1$ jet } & \multirow[b]{2}{*}{$f_{2}$} & \multicolumn{6}{|c|}{ Cross sections in pb for Higgs boson production with } \\
\hline & & & $\geq 2$ jets & $f_{3}$ & $\geq 3$ jets & $f_{4}$ & $\geq 4$ jets & $r_{n+1 / n}$ \\
\hline $\mathrm{H}+n$ jets & & & \multicolumn{3}{|c|}{$8 \mathrm{TeV} \mathrm{LHC}$} & \multicolumn{3}{|c|}{ @ LO (using NLO PDFs) } \\
\hline $\mathrm{H}+1$ jet & $3.020_{-28 \%}^{+44 \%}$ & 0.0 & & & & & & 0.308 \\
\hline $\mathrm{H}+2$ jets & & & $0.931_{-36 \%}^{+63 \%}$ & 0.0 & & & & 0.271 \\
\hline \multirow[t]{2}{*}{$\mathrm{H}+3$ jets } & & & & & $0.252_{-42 \%}^{+82 \%}$ & 0.0 & & \\
\hline & & & \multicolumn{3}{|c|}{$8 \mathrm{TeV} \mathrm{LHC}$} & & & @ NLO \\
\hline $\mathrm{H}+1$ jet & $5.096_{-17 \%}^{+17 \%}$ & 0.183 & $0.930_{-36 \%}^{+63 \%}$ & & & & & 0.292 \\
\hline $\mathrm{H}+2$ jets & & & $1.490_{-21 \%}^{+17 \%}$ & 0.169 & $0.252_{-42 \%}^{+82 \%}$ & & & 0.269 \\
\hline $\mathrm{H}+3$ jets & & & & & $0.401_{-25 \%}^{+17 \%}$ & 0.157 & $0.063_{-47 \%}^{+101 \%}$ & $(0.157)$ \\
\hline$K_{n}$ & 1.69 & & 1.60 & & 1.59 & & & \\
\hline $\mathrm{H}+n$ jets & & & $13 \mathrm{TeV}$ & $\mathrm{LHC}$ & & @ I & $\mathrm{O}$ (using NL & ( PDFs) \\
\hline $\mathrm{H}+1$ jet & $7.968_{-26 \%}^{+38 \%}$ & 0.0 & & & & & & 0.371 \\
\hline $\mathrm{H}+2$ jets & & & $2.954_{-34 \%}^{+58 \%}$ & 0.0 & & & & 0.329 \\
\hline $\mathrm{H}+3$ jets & & & & & $0.972_{-40 \%}^{+76 \%}$ & 0.0 & & \\
\hline & & & $13 \mathrm{TeV}$ & $\mathrm{LHC}$ & & & & @ NLO \\
\hline $\mathrm{H}+1$ jet & $13.19_{-15 \%}^{+15 \%}$ & 0.288 & $2.953_{-34 \%}^{+58 \%}$ & & & & & 0.341 \\
\hline $\mathrm{H}+2$ jets & & & $4.5000_{-18 \%}^{+13 \%}$ & 0.216 & $0.971_{-40 \%}^{+76 \%}$ & & & 0.319 \\
\hline $\mathrm{H}+3$ jets & & & & & $1.437_{-22 \%}^{+11 \%}$ & 0.206 & $0.296_{-45 \%}^{+94 \%}$ & $(0.206)$ \\
\hline$K_{n}$ & 1.66 & & 1.52 & & 1.48 & & & \\
\hline
\end{tabular}

Table 2. The $8 \mathrm{TeV}$ (upper half) and $13 \mathrm{TeV}$ (lower half) LHC cross sections in pb for the various parton-level Higgs boson plus jet samples used in this study. The cross sections have been obtained utilizing scale choice B (i.e. all scales are evaluated at $\hat{H}_{T}^{\prime} / 2$ ). For each center-of-mass energy, the upper and lower parts of the subtables show the LO and NLO results, respectively, together with their uncertainties (in percent) from varying scales by factors of two, up (subscript position) and down (superscript position). The NLO-to-LO $K$-factors, $K_{n}$, for the inclusive 1 -jet $(n=1)$, 2-jets $(n=2)$ and 3 -jets $(n=3)$ bin, the cross section ratios $r_{2 / 1}, r_{3 / 2}$ and $r_{4 / 3}$ as well as the $m$-jet fractions, $f_{m}$, are given in addition. Note that for the NLO calculations, $f_{4}$ and $r_{4 / 3}$ coincide as the predictions for $\mathrm{H}+4$ jets are only $\mathrm{LO}$ accurate. 

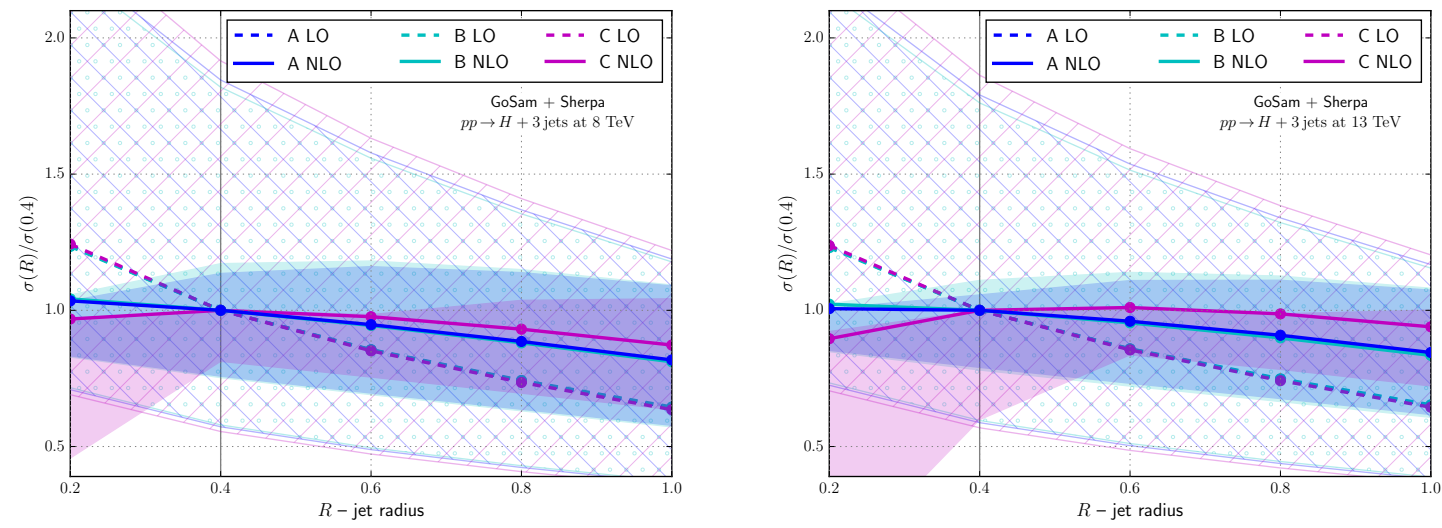

Figure 2. Total $\mathrm{LO}$ and $\mathrm{NLO}$ cross sections for $\mathrm{H}+3$ jets production as a function of the jet radius $R$ normalized to the corresponding rates for $R=0.4$. The left plot shows the results for $8 \mathrm{TeV}$, while the right one shows those obtained at $13 \mathrm{TeV}$ center-of-mass energy. Each plot displays the predictions for the three scale choices given in eqs. (2.8).

we only need to consider how easy it is to lose a (third) jet. Each real emission contribution comes with an additional parton in the final state. The calculation of these corrections will hence be based on an ensemble of partons that on average carries softer partons than those entering the $\mathrm{LO}$ calculation. For small values of $R$, the partons will not be clustered, i.e. there may be an increased probability that the event will not pass the selection criteria because there are not enough jets above the $p_{T}$ threshold. However, at least two partons have to be lost for this scenario to be true, and so the effect may be washed out. If the jet radius is increased, partons that are relatively close in $R$-space, but where each of them is below the jet threshold, get clustered together and can form a jet. If the radius is increased even further, we finally end up with the same situation that we have found at LO, i.e. decreasing cross sections for rising values of $R$. On the one hand, we therefore expect a change in the slope of the $R$ dependence peaking at medium $R$ values. On the other hand, the interplay between the LO-like behavior of the Born, virtual etc. results with the one of the real emission contribution leads to an overall stabilization of the $R$ dependence. Comparing the results for different scale choices, we observe rather small effects in general, but one has to bear in mind that the plots in figure 2 essentially display ratios; thus, differences due to different runnings and factors of $\alpha_{\mathrm{s}}$ cancel out to large extent.

To test another important parameter in our calculations, figure 3 is used to visualize the scale dependence of the total cross section for different PDF sets and different scale choices. The plots show results obtained for scale A, B and C, and for three different PDF sets, CT10nlo [119], MSTW08 [121] and NNPDF23 [122]. For scale C, we restrict ourselves to show the result for one PDF set only. The upper and lower panels are used to present the results of $8 \mathrm{TeV}$ and $13 \mathrm{TeV}$ collisions, respectively, while to the left and right of the figure we distinguish the two cases of $\mathrm{H}+2$ jets and $\mathrm{H}+3$ jets production.

Independent of the jet multiplicity, we observe the typical change of shape when going from LO to NLO. Both processes have their maximum approximately at half of the central 

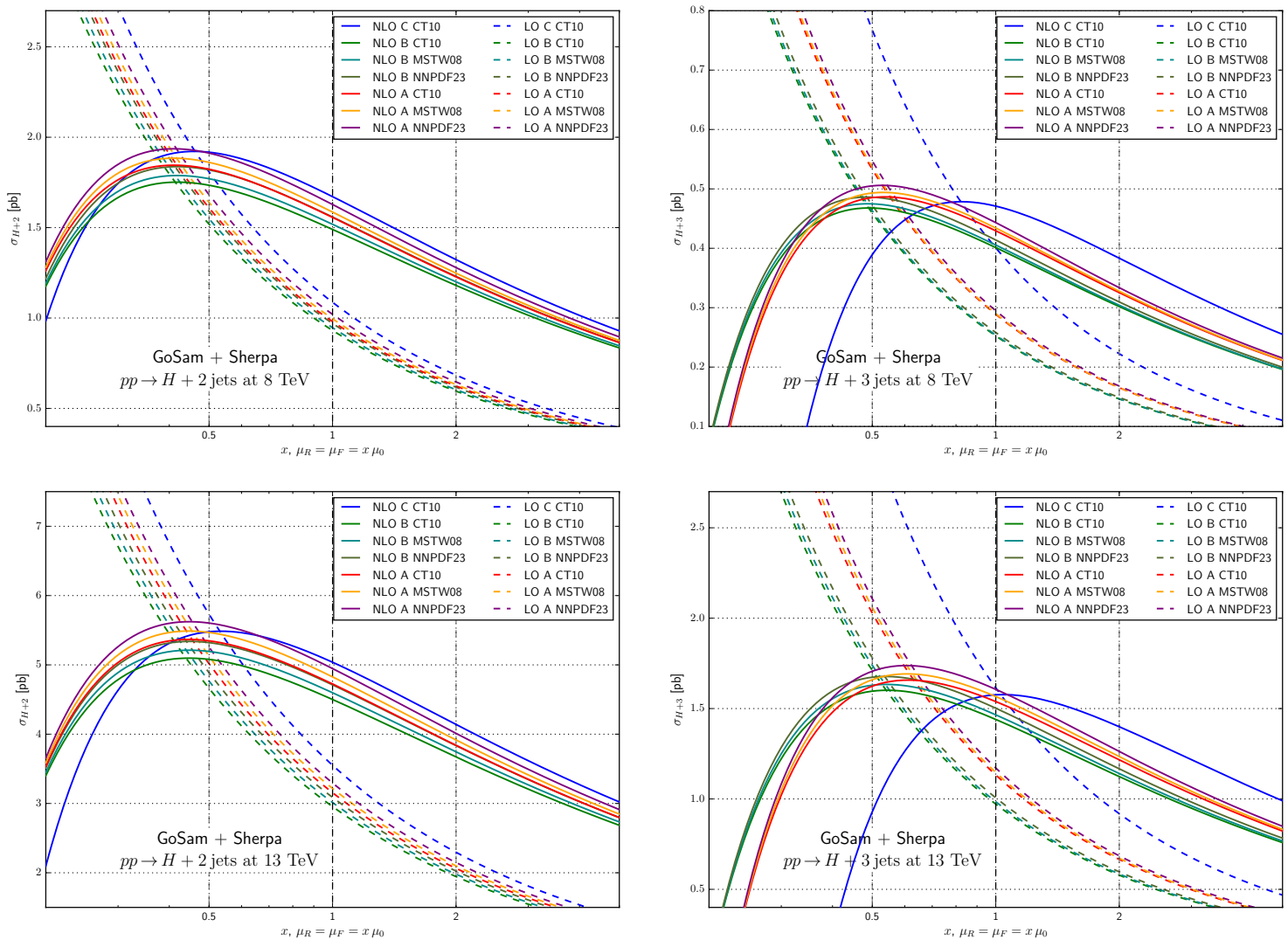

Figure 3. Scale dependence of the total cross section for scale choices A, B and C, and for different PDF sets. Results are shown for a center-of-mass energy of $8 \mathrm{TeV}$ (upper row) and $13 \mathrm{TeV}$ (lower row), and for the production of $\mathrm{H}+2$ jets (left column) and $\mathrm{H}+3$ jets (right column).

scale, except for scale $\mathrm{C}$ in the 3-jet case, whose maximum is shifted towards higher values. This means that taking the central scale and varying it up and down by a factor of two yields a reliable estimation of the theoretical uncertainties. Although the different PDF sets lead to slightly different results, their effect is considerably smaller than a different choice of the scale. If we compare the 2-jet and 3-jet results, we see that for the former the variation of scales and PDFs leads to a broader range of results, i.e. the spread between the single curves is larger compared to the 3-jet case, where the curves (except that for scale C) seem to be bundled more closely. Scale C, the Higgs boson mass scale, is smaller than the other two scales; the curves are hence shifted to higher values of the scaling parameter $x$. For the 2 -jet process, scale $\mathrm{C}$ is still in quite good agreement with the other scales, which is clearly not the case for the 3-jet process. Another interesting point is that for both jet multiplicities, the shapes are almost independent of the center-of-mass energy; the plots for $8 \mathrm{TeV}$ and $13 \mathrm{TeV}$ are very similar. Only for scale choice $\mathrm{C}$, we find a visible deviation, which is not surprising as it is a fixed scale and therefore does not account for a change in the center-of-mass energy.

Exclusive cross sections. Figure 4 shows the exclusive jet cross sections of the Higgs boson plus one-jet, two-jet and three-jet calculations for both the 8 and $13 \mathrm{TeV}$ collider 

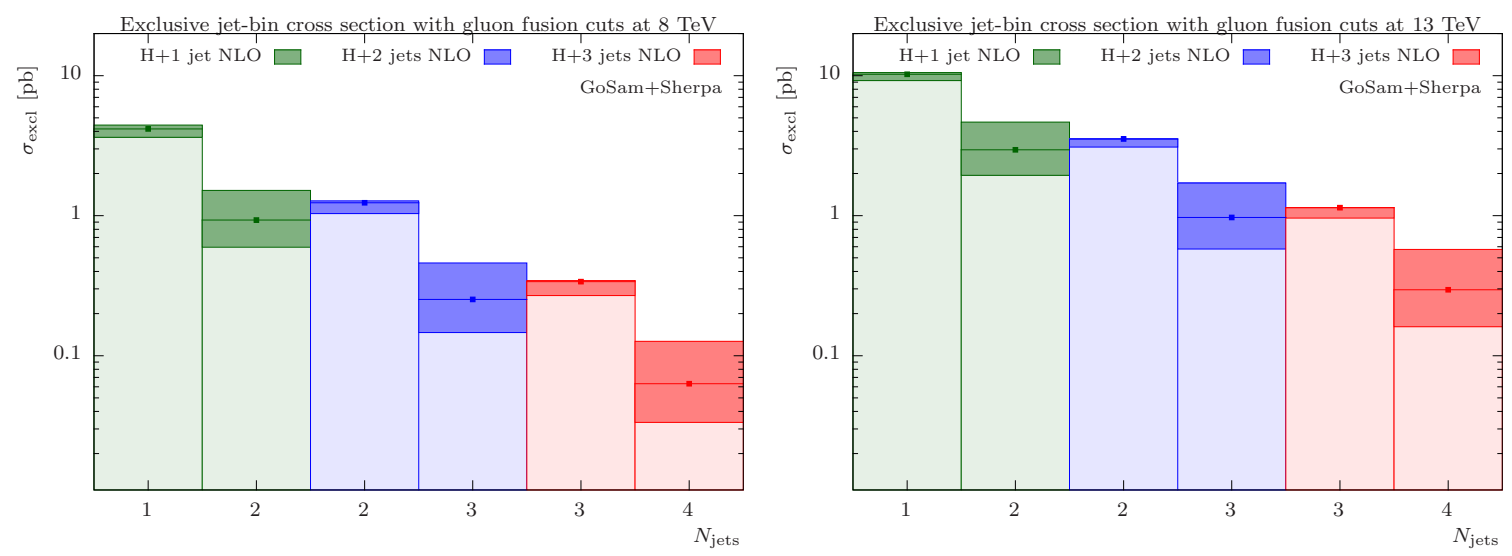

Figure 4. Exclusive jet cross sections (using the default scale choice and $R$ parameter) for the production of $\mathrm{H}+n$ jets $(n=1,2,3)$ at LHC energies of $8 \mathrm{TeV}$ (left) and $13 \mathrm{TeV}$ (right). For each of the three processes, two bins are plotted: the left-hand ones contain the respective exclusive NLO contribution, while the right-hand ones exhibit the respective contribution to the $(n+1)$-jet final state, i.e. the real emission contribution described with LO accuracy. The darker shaded areas indicate the size of the uncertainty obtained from scale variations.

energy of the LHC. At NLO, a $\mathrm{H}+n$ jets process contributes, of course, to two jet multiplicities, to the $n$-jet bin as well as to the $(n+1)$-jet bin. The contribution to the $n$-jet bin is given with NLO accuracy, whereas the $(n+1)$-jet contribution can only be LO accurate, as it is given by the real emission contribution. For each jet multiplicity, the left-hand bin therefore contains the exclusive $n$-jet contribution, while the right-hand bin displays the respective $(n+1)$-jet contribution. As we have utilized the same set of NLO PDFs for both the LO and NLO calculations, the real emission contribution of the $n$-jet process is exactly equal to the LO prediction for the $(n+1)$-jet process - and hence there is no need to display the LO results separately. The shaded areas of the different jet bins are used to illustrate the theoretical uncertainties stemming from the scale variation. As the contribution to the $(n+1)$-jet bin is only given at LO accuracy, the error bars are substantially larger compared to the $n$-jet bin depicting the respective prediction. It is important to mention that for each of the three different jet final states, the respective $(n+1)$-jet contribution constitutes a substantial fraction of the total cross section. This is particularly important when investigating observables that separate the two contributions, as in the case of vetoed cross sections. We will return to this point in section 4, when dealing with VBF topologies.

NLO effects in the spotlight. To validate the outcome of new NLO calculations, one should always inspect the types of observables that by construction will be hugely impacted by the occurrence of the NLO corrections. Most prominent examples are variables that are exclusively sensitive to the real emission contributions, such as the distribution of the transverse recoil momenta associated with the Born system. In our case, one interesting class of observables is defined by constructing the combined momenta of the Higgs boson and the two leading- $p_{T}$ jets. Observables operating on this 3-particle object, $\mathrm{H} j_{1} j_{2}$, will be guaranteed to have direct sensitivity to any additional radiation beyond the two leading 


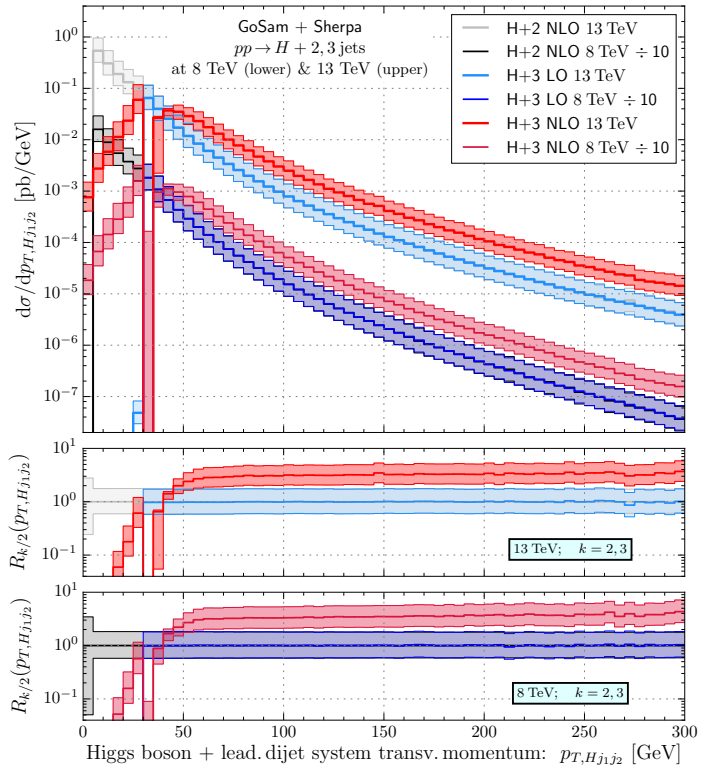

(a)

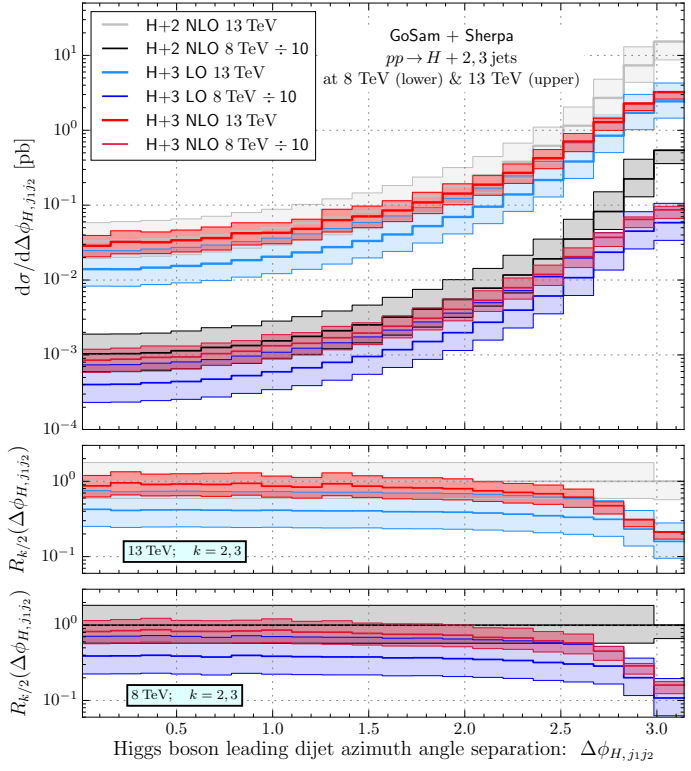

(b)

Figure 5. The transverse momentum distribution of the combined Higgs boson plus associated leading dijet system (5a), and the distribution of the relative azimuthal angle between them, $\Delta \phi_{\mathrm{H}, j_{1} j_{2}},(5 \mathrm{~b})$ at both LHC center-of-mass energies of $13 \mathrm{TeV}$ as well as $8 \mathrm{TeV}$. Predictions for $\mathrm{H}$ +3 jets production and $\mathrm{H}+2$ jets at NLO are compared with each other. Envelopes are used to illustrate the effect of standard scale variations by factors of two. Ratio plots underneath the main panel are shown separately for both collider energies.

jets. They will also have benefits when studying VBF topologies since one starts out from at least two jets in the final state.

In this subsection, we will present a few of these observables where the NLO effects manifest themselves clearly. The discussion will be technical as it centers on explaining the (partly very) different behavior of the NLO predictions for the various jet multiplicities. We start by showing in figure 5 a the transverse momentum distribution of the $\mathrm{H} j_{1} j_{2}$ system as introduced above. For both collider energies, we include the predictions from the $\mathrm{H}+$ 3 jets $\mathrm{LO}$ and $\mathrm{NLO}$ calculation as well as the $\mathrm{H}+2$ jets NLO calculation. The latter also serves as the reference in the ratio plots, which we give separately for each $E_{\mathrm{cm}}$ in the lower part of the figure. To better disentangle the $p_{T, \mathrm{H} j_{1} j_{2}}$ curves related to different energies, we have divided the distributions for $8 \mathrm{TeV}$ by a factor of 10 in the main plot. Relying on scale choice $\mathrm{B}$, the bands around the central predictions reflect the size of the uncertainties due to standard scale variations by factors of two. For the ratio plots, this means that the $\mathrm{LO}$ and NLO H +3 jets curves - the central ones as well as the scale-varied ones - have been normalized to the NLO $\mathrm{H}+2$ jets prediction obtained from the central scale choice. ${ }^{1}$

\footnotetext{
${ }^{1}$ To indicate the magnitude of the uncertainty, the ratio plots, in addition, contain the scale-varied predictions of the reference curve, i.e. the $\mathrm{NLO} \mathrm{H}+2$ jets prediction in figure $5 \mathrm{a}$.
} 
Thus, more generally, we can express these quantities in the ratio plots by

$$
R_{k / n}(O)=\frac{\frac{\mathrm{d} \sigma}{\mathrm{d} O}(\mathrm{H}+k \text { jets })}{\frac{\mathrm{d} \sigma}{\mathrm{d} O}(\mathrm{H}+n \text { jets })},
$$

which essentially provides us with a differential form of the ratio definition given in eq. (3.1). We note that this type of ratio plot will be used quite often throughout the paper. For the case at hand, $k=2,3$ while $n=2$ selecting the $\mathrm{H}+2$ jets $\mathrm{NLO}$ central prediction obtained from scale choice B.

We are using the same PDF set for both the LO and NLO calculations, hence the $\mathrm{NLO} \mathrm{H}+2$ jets and the $\mathrm{LO} \mathrm{H}+3$ jets curves in figure 5 a are identical above the jet $p_{T}$ threshold. They predict the transverse momentum spectrum of a third parton at LO. In the $\mathrm{H}+3$ jets case, the jet $p_{T}$ threshold at $30 \mathrm{GeV}$ is made explicit. In the $\mathrm{H}+2$ jets sample instead, the third parton can become arbitrarily soft, leading to a distribution, which diverges for $p_{T, \mathrm{H} j_{1} j_{2}} \rightarrow 0$. The divergence is canceled by the virtual corrections that contribute to the first bin of the spectrum only.

In contrast to the $p_{T, \mathrm{H} j_{1} j_{2}}$ LO predictions discussed above, the $\mathrm{H}+3$ jets NLO calculation produces a spectrum, which exhibits additional important features: we observe the onset of what is known as Sudakov suppression around the jet $p_{T}$ threshold and below. We also notice a gap in the distribution right above the threshold, as well as large NLO $K$-factors at larger values of $p_{T, \mathrm{H} j_{1} j_{2}}$. Most of these features emerge as a result of the more complex final states underlying an NLO treatment of $\mathrm{H}+3$ jets. We thus note that universal parts of the virtual corrections in combination with kinematical effects in the radiative corrections lead to a depletion right above the threshold. Moreover, while for the Born and virtual contributions, the distributions for $p_{T, \mathrm{H} j_{1} j_{2}}$ and $p_{T, j_{3}}$ are exactly the same, for the real corrections, it is helpful to think in terms of the recoil $p_{T}$ that is generated by the vectorial sum of the third jet and the real radiation. Kinematical configurations with four jets or with three jets and an unresolved parton thus lead to $p_{T}$ enhancing as well as $p_{T}$ compensating effects such that we respectively find large, $\mathcal{O}(3)$, fairly constant corrections for higher $p_{T, \mathrm{H} j} j_{2}$ as well as a contribution to the spectrum below the jet $p_{T}$ threshold. We furthermore notice the rather small reduction of the scale uncertainties when comparing the $\mathrm{H}+3$ jets $\mathrm{LO}$ and NLO predictions. The latter is also seen to retain a fairly symmetric uncertainty band which is uncommon to many other $\mathrm{H}+3$ jets NLO distributions. ${ }^{2}$ Taken these observations they clearly reflect the omnipresence of the real radiation effects: both types of contributions the four-jet ones and the three-jet ones containing an unresolved parton are spread over the entire $p_{T}$ range and essentially feature a LO scale variation only. Consequently, the $p_{T, \mathrm{H} j_{1} j_{2}}$ spectrum remains to be a spectrum that is largely influenced by

\footnotetext{
${ }^{2}$ More explicitly, we note that the 'BVI' (Born, virtual and integrated subtraction term) contributions of the $p_{T, \mathrm{H} j_{1} j_{2}}$ and $p_{T, j_{3}}$ distributions are exactly the same while the 'RS' (subtracted real) ones of the former are considerably harder than those of the latter. This leads to different cancellation patterns when combining the scale-varied 'BVI' and 'RS' predictions (which work in opposite directions). For the $p_{T, \mathrm{H} j_{1} j_{2}}$, we then find the 'RS' uncertainties to be the dominating ones for $p_{T, \mathrm{H} j_{1} j_{2}} \gtrsim 60 \mathrm{GeV}$.
} 
the respective higher jet-multiplicity process - even for the case of $\mathrm{H}+3$ jets production at NLO. As this issue occurs more commonly, we will discuss it in more detail in section 3.4.

Figure 5b shows the azimuthal separation between the Higgs boson and the two leading transverse momentum jets, $\Delta \phi_{\mathrm{H}, j_{1} j_{2}}$. The peak at $\Delta \phi \sim \pi$ is common to all curves and indicates that the Higgs boson is preferably recoiling against the jet-jet system built by the two leading jets. In fact, this is the only possible kinematical configuration emerging in $\mathrm{H}+2$ jets production at LO. The spread to smaller values of $\Delta \phi_{\mathrm{H}, j_{1} j_{2}}$ therefore gives an estimate of the size of the higher-order corrections. Starting off with the $\mathrm{H}+2$ jets NLO prediction, it essentially gives a LO description of the $\Delta \phi$ variable. The associated curves correspondingly show a very large scale dependence over the full spectrum, the only exception being the bin at $\Delta \phi=\pi$, which contains the singular real emission configurations and the virtual corrections. In contrast to $p_{T, \mathrm{H} j_{1} j_{2}}$, this time there is no overlap between the $\mathrm{LO}$ predictions for $\mathrm{H}+3$ jets and the NLO results for $\mathrm{H}$ +2 jets. In the latter case, the soft (below jet threshold) real radiation component can still contribute to the spectrum at $\Delta \phi_{\mathrm{H}, j_{1} j_{2}}<3$, i.e. outside the $\Delta \phi=\pi$ bin. In other words, the gap between the $\mathrm{H}+2$ jets and $\mathrm{H}+3$ jets curves is filled by two-jet events that contribute to the $p_{T, \mathrm{H} j_{1} j_{2}}$ spectrum below $30 \mathrm{GeV}$. Turning to the NLO corrected three-jet case, we find that for both collider energies, the relative size of these corrections is more than a factor of 2 for $\Delta \phi_{\mathrm{H}, j_{1} j_{2}} \rightarrow 0$ and decreases to about $40 \%$ for $\Delta \phi_{\mathrm{H}, j_{1} j_{2}} \rightarrow \pi$.

We conclude this section by discussing the features of figure 6 , used to present two more technicalities that are of relevance to all Higgs boson plus jets final states. For the three different jet multiplicities considered here, figure 6a shows the azimuthal separation between the Higgs boson and the leading $p_{T}$ jet. Increasing the number of jets has a very dramatic effect on this observable. In fact in the $\mathrm{H}+1$ jet case, deviations from a pure back-to-back (i.e. LO) configuration are only made possible through real radiation. The distribution is therefore $\mathrm{LO}$ accurate only and possesses a large uncertainty band. For $\mathrm{H}+2$ jets production, the azimuthal separation in a Born-like event cannot fall below 90 degrees, because the Higgs boson and the leading jet cannot recoil together against a second, softer jet. However, the presence of a third jet originating from the real radiation contribution may enable such recoil configurations. It is for this reason that the $\mathrm{H}+2$ jets curve at NLO features a reduced uncertainty band for $\pi / 2<\Delta \phi_{H, j_{1}}<\pi$ and a larger, i.e. LO, uncertainty band for smaller angles. The large drop of the $\mathrm{H}+1$ jet curve for angles $\Delta \phi_{H, j_{1}}<\pi / 2$ can be explained in a similar way. Still there is one subtlety: the difference between the $\mathrm{LO} \mathrm{H}+2$ jets distribution that vanishes at $\Delta \phi_{H, j_{1}}=\pi / 2$ and the NLO $\mathrm{H}+1$ jet distribution arises as a result of real radiation events, which have their hardest emission appearing at very large pseudorapidities $(|\eta|>4.4)$. This hard parton cannot satisfy the applied jet cuts, but will certainly contribute to the $p_{T}$ balancing of the partonic final state. The associated $\mathrm{H}+1$ jet event will stand out as it carries a large missing transverse energy and helps populate the $\Delta \phi$ distribution below $\pi / 2$ eventually. In the $\mathrm{H}+3$ jets cases, this region of low angles is easily filled, and we obtain a definite reduction of the uncertainty band over the whole range of the distribution for $\mathrm{H}+3$ jets at NLO. Despite the fact that multiple jets are present in the final state, there still is a clear preference for the Higgs boson to recoil against the hardest jet. 


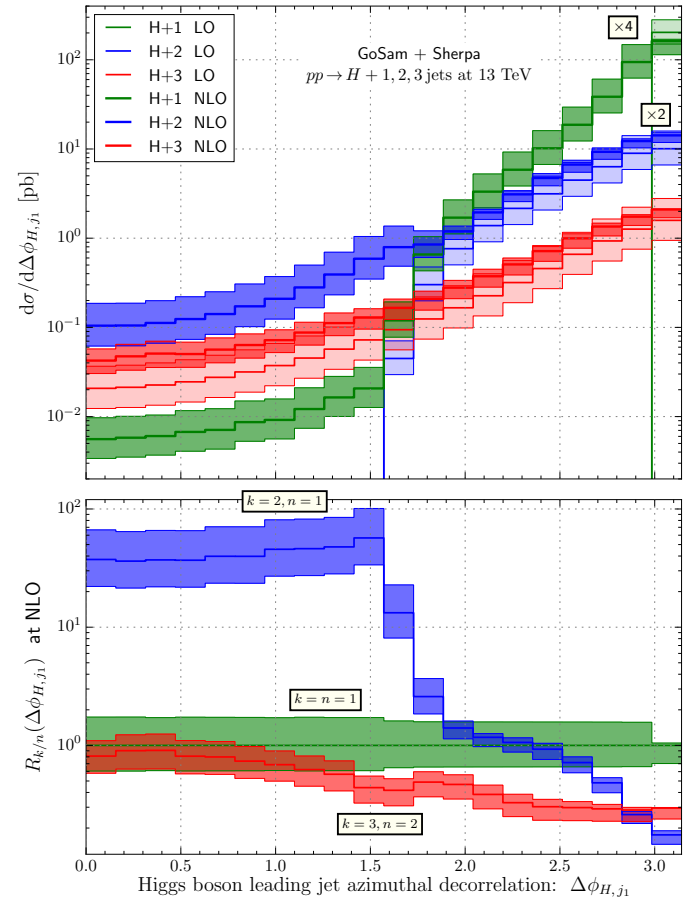

(a)

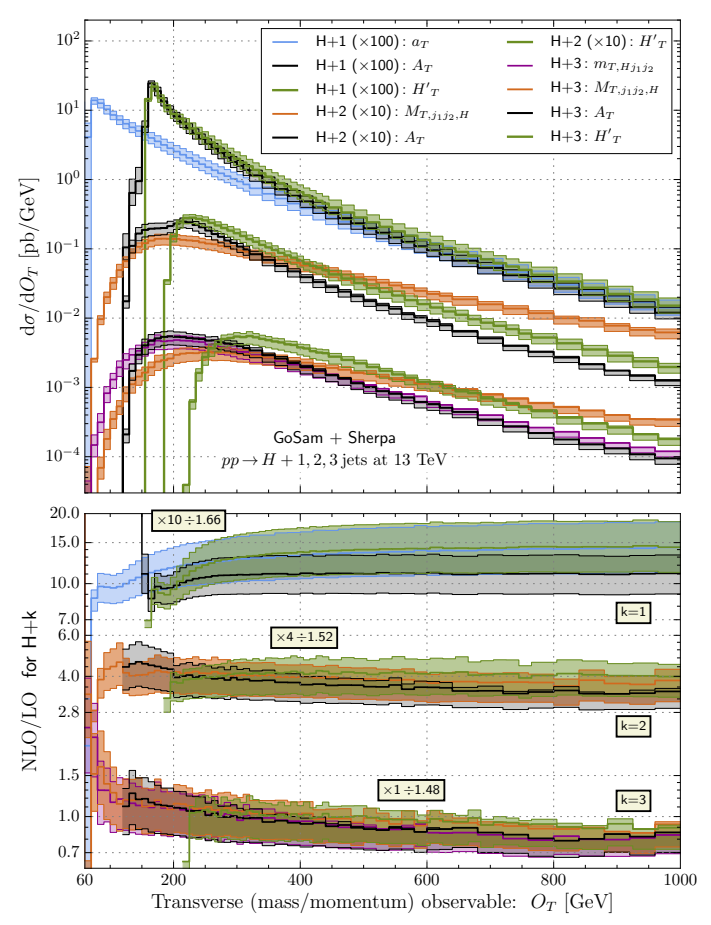

(b)

Figure 6. Azimuthal separation between the Higgs boson and the hardest jet (6a) for $\mathrm{H}+1$ jet, $\mathrm{H}+$ 2 jets and $\mathrm{H}+3$ jets production at $13 \mathrm{TeV}$ LHC center-of-mass energy. Note that the results for the $\mathrm{H}+1$ jet and $\mathrm{H}+2$ jets channels have been multiplied by additional factors of 4 and 2, respectively. While both the LO and NLO predictions are shown in the main plot, the lower subplot depicts differential ratios, $R_{k / n}$, between various $\mathrm{H}+$ jet cross sections at NLO only. Alternative transverse mass/momentum observables (6b) in comparison to the $H_{T}^{\prime}$ observable as derived from our default scale choice. NLO results are shown for all three $\mathrm{H}+n$ jets channels (separated by additional factors of 10) and an LHC collision energy of $13 \mathrm{TeV}$. For each observable, the differential $K$-factor is given in the associated ratio plot where again separation factors have been applied to enhance the visibility of the results.

As the $\hat{H}_{T}^{\prime}$ scale plays a central role in our calculations, we are interested in how it compares to other reasonable choices characterizing the transverse activity of Higgs boson occurrences in association with jets. Using the generalized transverse mass definitions,

$$
m_{T, 1 \ldots n}=\sqrt{H_{T, 1 \ldots n}^{2}-\left(\sum_{i} \vec{p}_{T, i}\right)^{2}}
$$

where $H_{T, 1 \ldots n}=\sum_{i=1}^{n} p_{T, i}$ and

$$
M_{T, A, B}=\sqrt{m_{A}^{2}+2\left(p_{T, B}\left(m_{A}^{2}+p_{T, A}^{2}\right)^{1 / 2}-\vec{p}_{T, B} \cdot \vec{p}_{T, A}\right)},
$$

given in ref. [123], we can construct observables that may serve as a proxy for alternative scale choices. At the level of observables, i.e. where jets rather than partons are used 
to build the variable, we can investigate to what extent the alternative choices lead to deviations from the $\hat{H}_{T}^{\prime}$ default. We then obtain at least a qualitative understanding of the possible size of theoretical uncertainties that arise from a variation of the scale's functional form. This theoretical scale dependence should be kept in mind as it may turn out to be important in the interpretation of $\mathrm{H}+n$ jets cross section measurements.

The results of this comparison are presented in the right panel of figure 6 for the three different jet multiplicity final states. The main plot shows the transverse observables including $H_{T}^{\prime}$ (as green curves) while the ratio plot depicts the NLO vs. LO $K$-factors in dependence on the respective observable. These $K$-factors are noticed to show a great amount of overlap supporting the fact that the higher-order corrections to the different observables lead to rather similar effects. As before the bands indicate the size of standard scale $\mathrm{B}$ variations by factors of two. Note that for better visibility, the $\mathrm{H}+1,2,3$ jets results are separated by additional factors of 10 (by factors of 10 and 4) in the main (ratio) plot. The differential $K$-factors of the ratio plot are moreover divided by their respective inclusive $\mathrm{H}+n$ jets $K$-factors. The black curves, $A_{T} \equiv M_{T, \mathrm{H}, j_{1} \ldots j_{n}}$, represent a choice whose threshold is always given by $m_{\mathrm{H}}$ and does not shift upwards with increasing jet multiplicity as it occurs for $H_{T}^{\prime}$. In the $\mathrm{H}+1$ jet case, the differences are small while for $\mathrm{H}+2,3$ jets, they are more pronounced as $A_{T}$ dives into the softer region and thus gives rise to softer tails. In the $\mathrm{H}+2,3$ jets cases, we also show the effects of choosing a more VBF-inspired $M_{T}$ scale (orange curves). Although the soft region is covered even more widely, $M_{T, j_{1} j_{2}, \mathrm{H}}$ provides much harder tails compared to $A_{T}$ with no overlap among them. Again, softer tails but similar coverage of the soft region can be achieved by switching to $m_{T, \mathrm{H} j_{1} j_{2}}$ (purple curve) which we only demonstrate for the case of $\mathrm{H}+3$ jets. For $\mathrm{H}+1$ jet events, an $m_{T}$ based choice such as $a_{T} \equiv m_{T, \mathrm{H} j_{1} \ldots j_{n}}$ (depicted by the lightblue shaded curve) would then offer a scale setting very similar to $H_{T}^{\prime}$ but neglecting the Higgs boson mass. This is depicted by the curve shaded in lightblue. Based on the overall behavior of the alternative scales apart from $M_{T, j_{1} j_{2}, H}$, these scale choices can be anticipated to yield $\mathrm{H}+$ jets cross sections that will be larger than the default ones. For some cases, the cross sections may even be outside the uncertainty range as the standard scale variation bands do not overlap in all cases. This is where further investigations will have to be carried out.

\subsection{Single-particle observables}

We now turn to the discussion of one-particle or one-jet observables. Figures $7 \mathrm{~b}-7 \mathrm{~d}$ show the transverse momentum distribution of the Higgs boson in the $\mathrm{H}+3$ jets process for the three different scale choices $\mathrm{A}, \mathrm{B}$ and $\mathrm{C}$ of eqs. (2.8), while figure $7 \mathrm{a}$ shows the results for the different scales normalized to the NLO result for scale A. The advantage of scale B is the flatness of the $K$-factor over the entire $p_{T}$ range. This supports our choice to make scale $\mathrm{B}$ the default scale. For the lower $p_{T}$ region up to $\sim 250 \mathrm{GeV}$, scale $\mathrm{C}$ seems to be a sensible choice as well. However, it completely breaks down for higher $p_{T}$, and the $K$-factor can even become negative. ${ }^{3}$ This different behavior of the scale definitions is more pronounced in figure 7a. For scale $\mathrm{C}$, the $\mathrm{LO}$ curve shows the opposite behavior compared to the NLO

\footnotetext{
${ }^{3}$ The behavior was found to be similar, for example, in $\mathrm{W}+3$ jets production [124].
} 


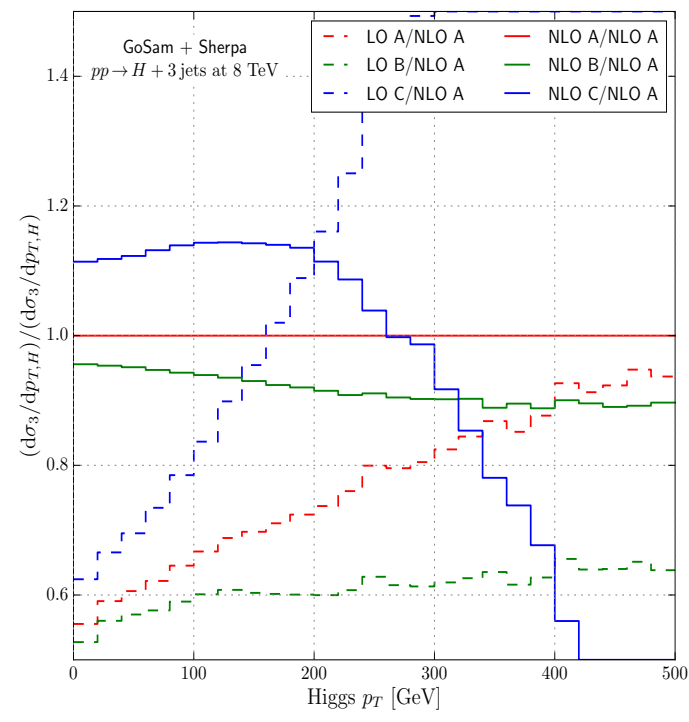

(a) Ratio.

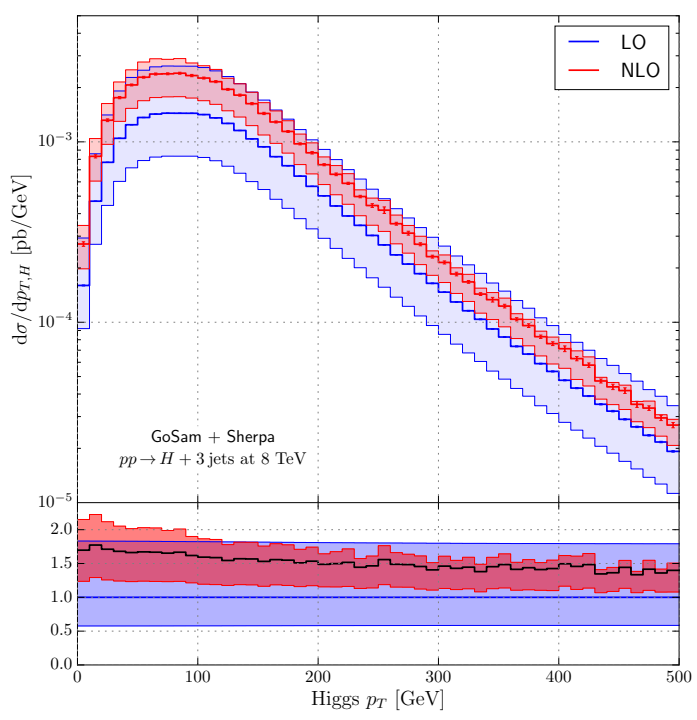

(c) Scale choice B (2.8b).

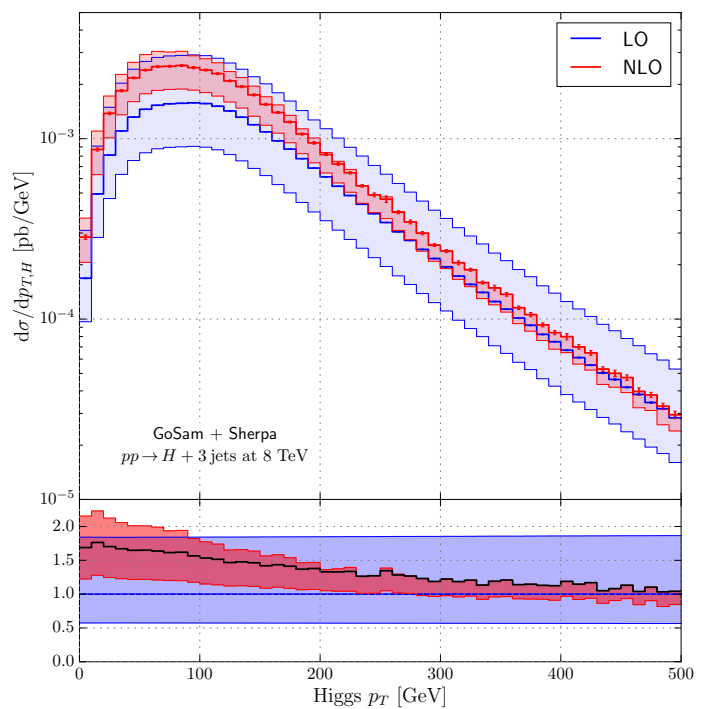

(b) Scale choice A (2.8a).

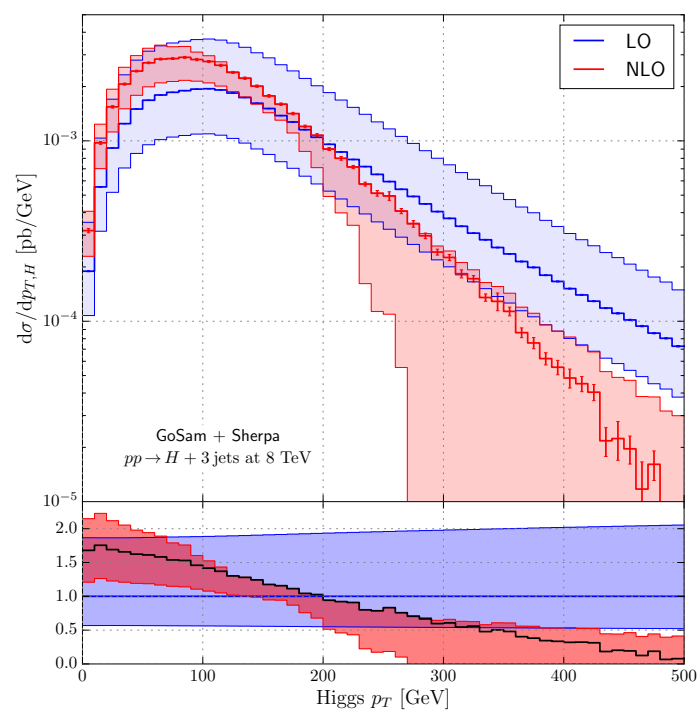

(d) Scale choice C (2.8c).

Figure 7. The $p_{T}$ distribution of the Higgs boson in $\mathrm{H}+3$ jets production at the $8 \mathrm{TeV}$ LHC presented for the three scales A, B and C of eqs. (2.8). The subplot 7a shows the same central predictions normalized to the NLO result for scale A. Each ratio plot depicts the respective differential $K$-factors and their envelopes obtained from scale variations at LO and NLO. 

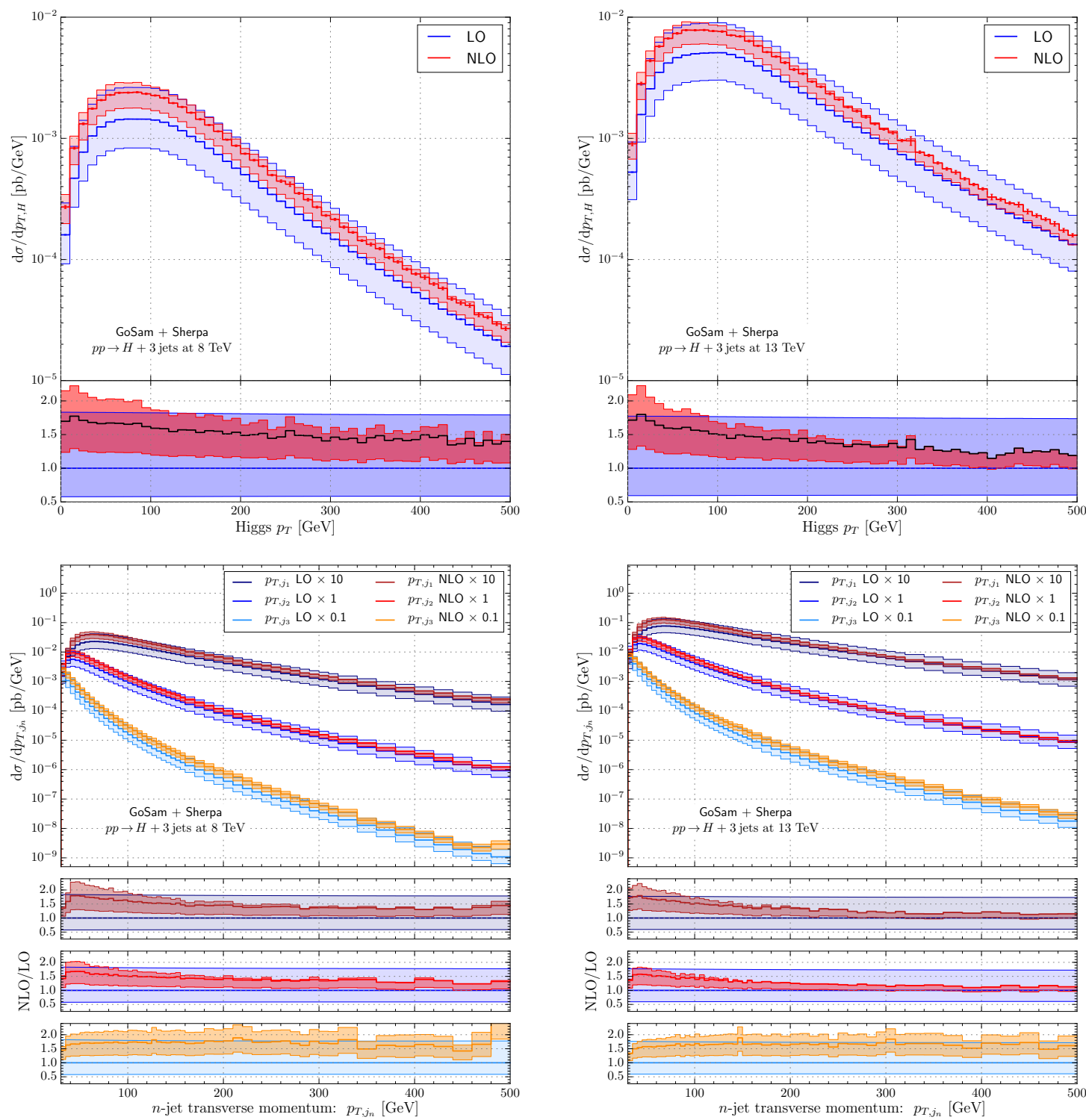

Figure 8. The $p_{T}$ distribution of the Higgs boson (upper row) and the three hardest jets (lower row) in $\mathrm{H}+3$ jets production at $E_{\mathrm{cm}}=8 \mathrm{TeV}$ (l.h.s.) and $E_{\mathrm{cm}}=13 \mathrm{TeV}$ (r.h.s.). Note that the $p_{T}$ distribution of the first and third jet has been rescaled by a factor of 10 and 1/10, respectively. Differential $K$-factors and their scale uncertainties are shown by the respective ratio plots.

curve. For scale A, the situation is fairly acceptable, but the $K$-factor still has a strong dependence on the $p_{T}$ of the Higgs boson. Selecting scale B, the latter improves nicely as for this choice, the LO and NLO curves are almost parallel, which is further confirmation that using $\hat{H}_{T}^{\prime}$ for all factors of $\alpha_{\mathrm{s}}$ is a sensible choice.

Utilizing scale choice $\mathrm{B}$, we compare in figure 8 the predictions for the $p_{T}$ distribution of the Higgs boson and the associated jets at LHC center-of-mass energies of $8 \mathrm{TeV}$ (left plots) and $13 \mathrm{TeV}$ (right plots). The upper row shows the LO and NLO result for the Higgs boson $p_{T}$. One observes that the NLO corrections only lead to a mild change of the shape, 
with a small decrease of the $K$-factor in the high- $p_{T}$ tail. Comparing the two center-of-mass energies, we also see that for both the LO and NLO curves the peak of the distribution remains in the region slightly below $p_{T} \sim 100 \mathrm{GeV}$. The distributions are steeper for $8 \mathrm{TeV}$, and the theoretical uncertainties of the NLO prediction at $13 \mathrm{TeV}$ are slightly smaller.

In the lower row, we show the transverse momentum distribution for the three leading $p_{T}$ jets. For a better visibility of the different curves, we have rescaled the first jet by a factor of 10 and the third jet by a factor of 0.1 . The second jet is shown unchanged. On a logarithmic scale, the rescaling only leads to a vertical shift of the different curves, preserving however their shapes and uncertainty envelopes. It therefore is possible to better distinguish the different behavior of the curves over the considered kinematical range. In the associated ratio plots, we show the 'NLO/LO' ratio for each pair of curves, i.e. each jet distribution has been normalized to its corresponding LO distribution. Looking at the distributions, we observe by and large a similar pattern as seen for the Higgs- $p_{T}$ : the peak of the distributions is almost insensitive to a change of the center-of-mass energy, but for the $8 \mathrm{TeV}$ results, the tail of the curves decreases faster. For the first and the second jet, one also obtains a slightly decreasing $K$-factor towards higher jet energies and a small reduction of the theoretical uncertainties when going from 8 to $13 \mathrm{TeV}$. The third jet however behaves somewhat differently. For each collider energy, the $K$-factor is almost flat and the size of the scale uncertainties remains constant towards higher $p_{T}$ values whereas for the first two jets, one obtains a slight reduction. This behavior of the third jet is driven by the size of the four-jet contribution featuring LO scale variation characteristics that yield wider uncertainty envelopes in general. Comparing the different $p_{T}$ spectra, the one of the third jet declines much faster towards higher $p_{T}$ values. This leads to an increase in the relative importance of the four-jet component retaining the rather uniform uncertainty band. It should be noted here that the transverse momentum spectra of figures 7 and 8, and in particular that of the Higgs boson, receive sizeable corrections when finite top-quark masses are taken into account $[86,125]$.

Another important observable is the rapidity. Figure 9 shows examples of distributions, for the Higgs boson (top row) and the three hardest jets (bottom), again for both collider energies of $8 \mathrm{TeV}$ (left column) and $13 \mathrm{TeV}$ (right column). Starting with the $y_{\mathrm{H}}$ spectrum, we first of all observe a very flat $K$-factor across the whole range of the distribution. Comparing the $8 \mathrm{TeV}$ with the $13 \mathrm{TeV}$ result, this holds true in both cases. In addition, we notice a mild reduction of the scale uncertainty for the $13 \mathrm{TeV}$ result, similarly to what we already observed for the $p_{T}$ distributions. However, the shape of the distribution changes for both the LO and NLO predictions when increasing the center-of-mass energy. For $E_{\mathrm{cm}}=8 \mathrm{TeV}$, the fraction of Higgs particles in the central region is higher and we see a steeper decline of the cross section towards larger values of the rapidity. This is much less pronounced for the $13 \mathrm{TeV}$ case where we obtain a relative enhancement of the large-rapidity regions.

For the rapidity distributions of the jets in the lower part of figure 9 , we have applied the same rescaling procedure as for the corresponding $p_{T}$ distributions in order to obtain a better readability of the plots. To very good approximation, these jet rapidities also feature a rather flat $K$-factor combined with a small reduction of the scale uncertainties when going from 8 to $13 \mathrm{TeV}$. The scale uncertainty bands are rather symmetric and steady, which is an indication for much more uniformly distributed four-jet effects, 

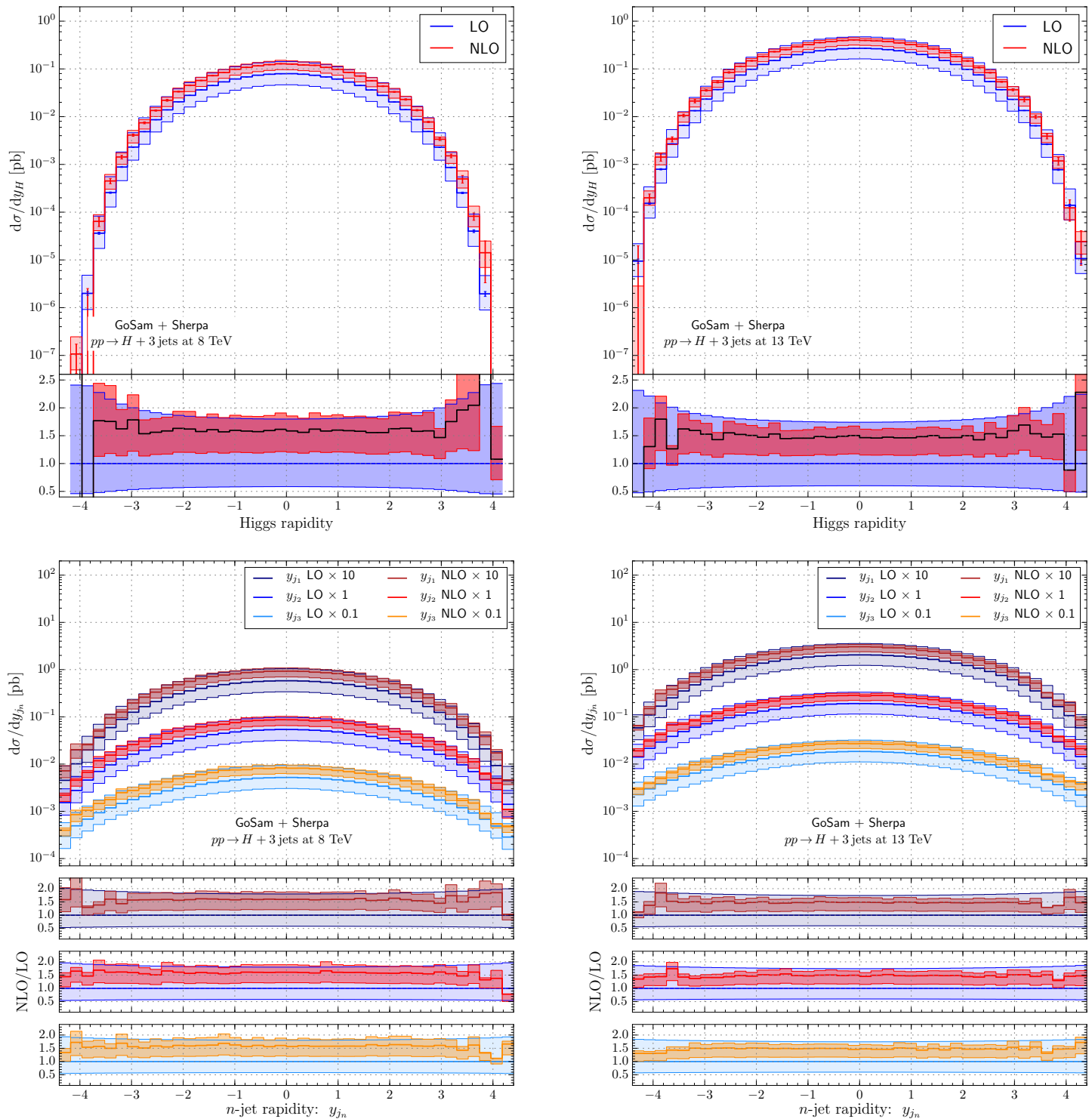

Figure 9. The rapidity distribution of the Higgs boson (upper row) and the three hardest jets (lower row) in $\mathrm{H}+3$ jets production at $E_{\mathrm{cm}}=8 \mathrm{TeV}$ (l.h.s.) and $E_{\mathrm{cm}}=13 \mathrm{TeV}$ (r.h.s.). Again, the rapidity curves for the first and the third jet have been rescaled by a factor of 10 and 1/10, respectively. Ratio plots are used to show differential $K$-factors and their scale uncertainty.

unlike previously discussed. As for the Higgs boson rapidities, in the $13 \mathrm{TeV}$ result, one can observe a relative enhancement of the regions with large rapidities. This is due to the fact that the increase in center-of-mass energy enables the production of more forward/backward scatterings of particles that satisfy the $p_{T}$ requirements on the jets at the same time. The corresponding phase space regions are thus populated more often, yielding wider rapidity distributions for the jets.

We conclude this section by discussing the impact of higher-order corrections on the 'wimpiest' jet in $\mathrm{H}+1$ jet, $\mathrm{H}+2$ jets and $\mathrm{H}+3$ jets production at $E_{\mathrm{cm}}=13 \mathrm{TeV}$. In 

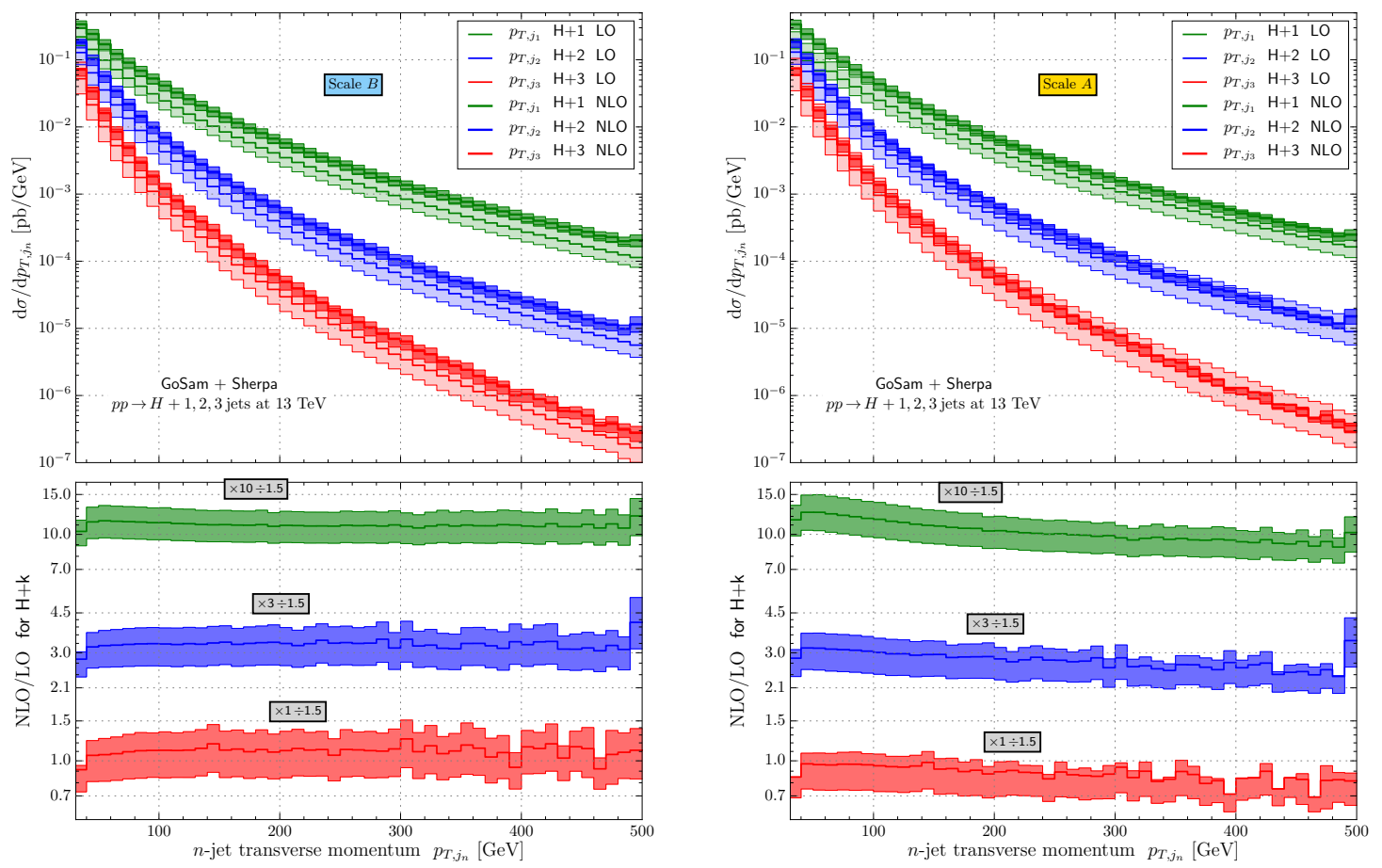

Figure 10. Transverse momentum distribution of the 'wimpiest' jet in $\mathrm{H}+n$ jets production at the LHC. Using $p_{T}$ ordering the first, second and third leading jet are shown in $\mathrm{H}+1$ jet, $\mathrm{H}+2$ jets and $\mathrm{H}+3$ jets at $13 \mathrm{TeV}$, respectively; on the left with the default scale choice $\mathrm{B}$, on the right with the scale choice A. The ratio plots depict the NLO vs. LO differential $K$-factors, appropriately rescaled for each jet multiplicity.

NLO calculations for $\mathrm{W} / \mathrm{Z}+$ jets performed with scale $\mathrm{B}$, it was noted that the transverse momentum spectrum of this jet exhibits a flat $K$-factor [126]. We test for the effect in $\mathrm{H}+$ jets production for the first time, and we find a similar behavior, as exemplified in the the left panel of figure 10. The green curves show the first jet in $\mathrm{H}+1$ jet, the blue ones the second jet in $\mathrm{H}+2$ jets, and the red ones the third jet in $\mathrm{H}+3$ jets production. The ratio plots show the transverse momentum dependent $K$-factors for these three cases, scaled by factors of $20 / 3(\mathrm{H}+1$ jet$), 2(\mathrm{H}+2$ jets $)$, and $2 / 3(\mathrm{H}+3$ jets $)$. It is evident that the $K$-factors are not only flat over the entire range, but they are also very similar for all three calculations. Figure 10, to the right, then shows the same analysis for the scale choice A. In this case the $K$-factors have a larger transverse momentum dependence as they are decreasing in the high- $p_{T}$ tail. Their scale uncertainty envelopes are less symmetric around the central predictions, which becomes more apparent for increasing jet multiplicities.

\subsection{Multi-particle observables and correlations}

Multi-particle or multi-jet observables are at the core of any measurement that involves many objects in the final state. They allow to test QCD dynamics at the LHC to an unprecedented precision, and they often reveal inappropriate modeling by LO calculations or by MC event generators. 

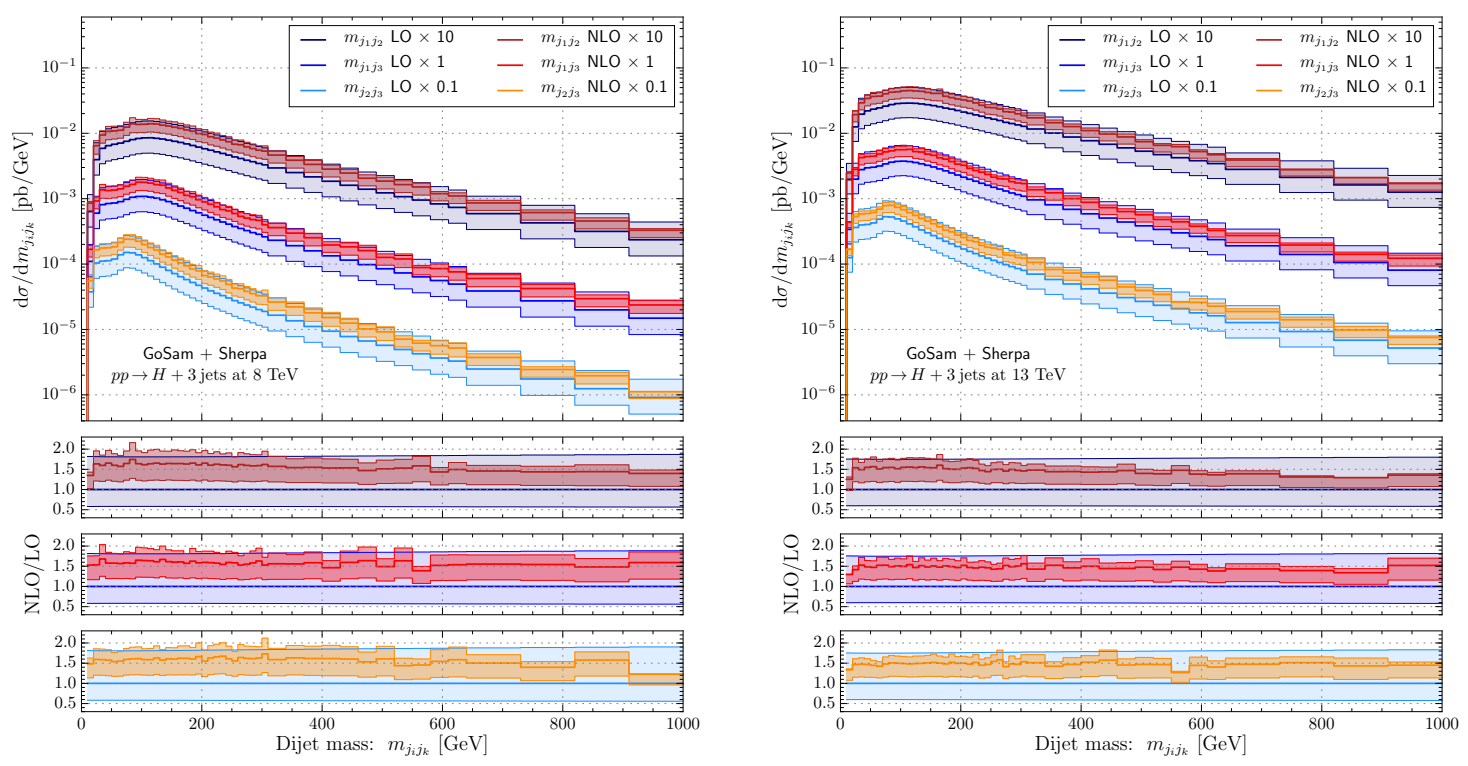

Figure 11. Invariant mass distribution for the dijet systems combined from the three hardest jets in $\mathrm{H}+3$ jets production. Results are shown for $8 \mathrm{TeV}$ (1.h.s) and $13 \mathrm{TeV}$ (r.h.s). Jets are ordered in $p_{T}$. Each ratio plot contains the differential $K$-factor and its scale uncertainty envelope for the respective jet-jet combination.

Figure 11 shows the dijet invariant mass distribution, $m_{j_{i} j_{k}}$, for each of the three possible combinations: $(i, k) \in\{(1,2),(1,3),(2,3)\}$ where the jets are ordered in transverse momentum. The left panel shows results for $E_{\mathrm{cm}}=8 \mathrm{TeV}$, while the right one shows them for $E_{\mathrm{cm}}=13 \mathrm{TeV}$. In order to avoid overlapping distributions in the figure, the curves for $m_{j_{1} j_{2}}$ are rescaled by a factor of 10 , whereas those for $m_{j_{2} j_{3}}$ are rescaled by a factor $1 / 10$. We notice a steeper decrease of the distributions in the $8 \mathrm{TeV}$ case and also for combinations of softer jets, as expected. Comparing the left and right panels, one observes that the maximum of the curves is to a good approximation independent of the collider energy.

In the lower part of the plots, we show separately the $K$-factors for the three distributions. Apart from the expected reduction of the theoretical uncertainty, we observe a $K$-factor that is approximately constant for both energies and for all the three jet combinations. As for the $p_{T}$ distributions, the two hardest jets are less influenced by specific four-jet effects. Hence, only the invariant mass of the two leading jets, $m_{j_{1} j_{2}}$, shows a small decrease in the relative size of the NLO corrections and uncertainty bands for higher values, in particular at $13 \mathrm{TeV}$. This is of course to a large extent due to the scale choice.

A further observable that is particularly important in view of vector boson fusion processes is the azimuthal angle $\Delta \phi_{j_{i}, j_{k}}$ between jets, as shown in figure 12. Again, we give predictions for the three different pairs of hardest- $p_{T}$ jets at both energies $8 \mathrm{TeV}$, on the left, and $13 \mathrm{TeV}$, on the right. As for the invariant masses (and more so the rapidities discussed above), one observes a flat $K$-factor for all combinations and for both energies. The shape however changes slightly when increasing the energy. This is particularly visible in the peak regions, which are more pronounced at $13 \mathrm{TeV}$. Their position is related to the 

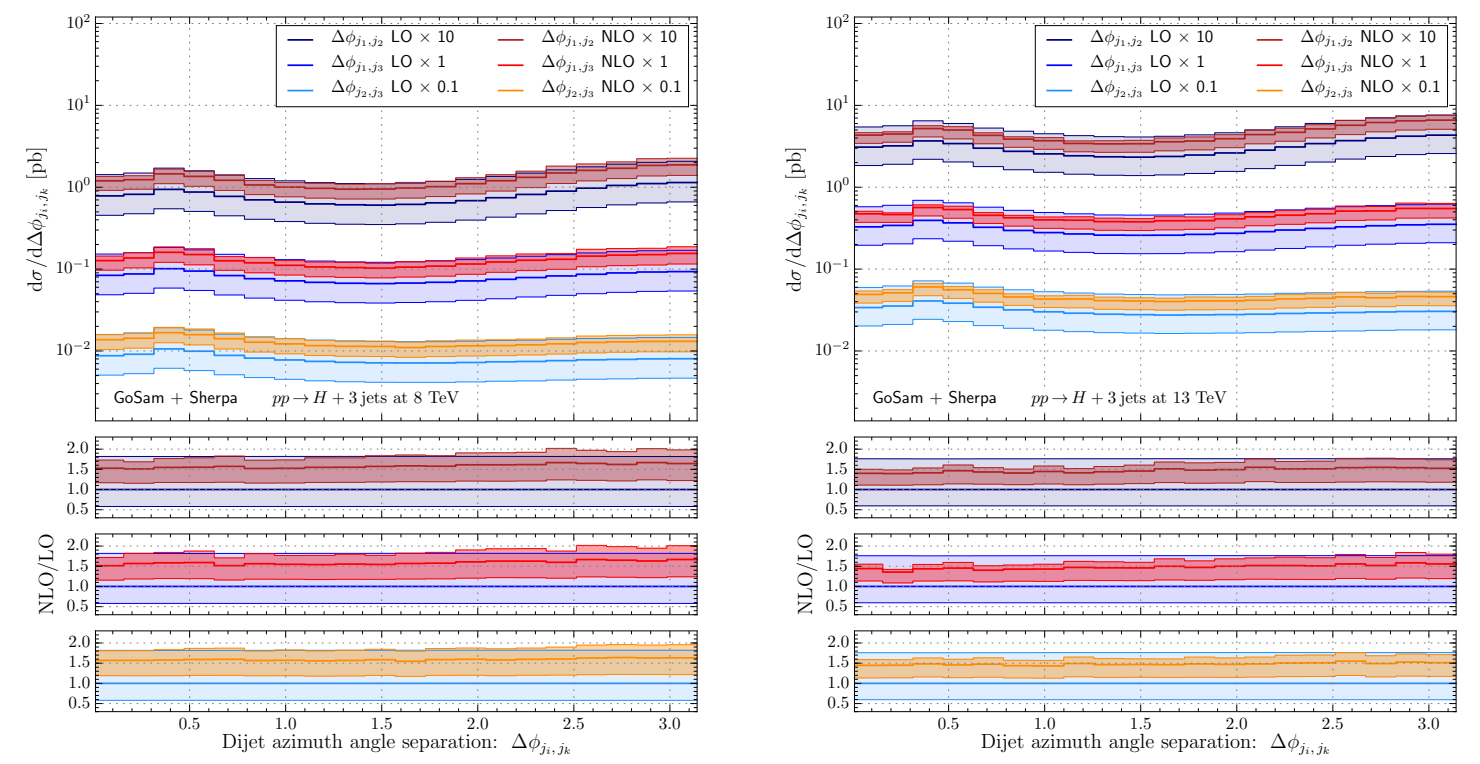

Figure 12. Distribution of the azimuthal angle $\Delta \phi$ between the two jets forming a dijet system. Predictions are shown for the three hardest- $p_{T}$ jet combinations at collider energies of $8 \mathrm{TeV}$ (1.h.s.) and $13 \mathrm{TeV}$ (r.h.s.). For each jet-jet combination, one ratio plot is added containing the differential $K$-factor and its corresponding scale uncertainty envelope.

choice of the jet radius $R$ (per default $R=0.4$ ). In particular for configurations where the two jets have $\Delta y_{j_{i}, j_{k}}=0$, the $\phi$ separation exactly corresponds to the chosen value of $R$.

Further multi-particles observables, less related to multi-jet QCD dynamics and more specific to the Higgs boson production in association with jets have been already shown before in section 3.1. For more details, see the discussion around figures 5 and 6 .

\subsection{Multi-jet ratios at NLO}

In this section, we ask the question how observables change in the presence of additional QCD radiation, starting with a core process specified by $\mathrm{H}+n_{\min }$ jets. Here, we mainly rely on using $n_{\min }=1$ as the $\mathrm{H}+1$ jet process represents our most inclusive case. The VBF topology requires at least two jets, but our observations hold in both cases, largely because the phase space available to QCD radiation at the LHC is tremendous. This has been pointed out many times before, and a particularly nice example of the effects is given in ref. [127]. In this section, we use our NLO results for $\mathrm{H}+n$ jets $(n=1,2,3)$ to make some of the statements explicit.

The visible energy, $H_{T}^{\prime}$, is the classical example of a 1 -jet inclusive observable, which is impacted by higher-order radiative effects, simply because it sums the Higgs boson $p_{T}$ and all jet transverse momenta, irrespective of their correlations in azimuth. The corresponding spectrum is shown in figure 13 for an LHC energy of $8 \mathrm{TeV}$ (left column) and $13 \mathrm{TeV}$ (right column). The upper plots show the NLO distributions for one, two and three jets (which we have obtained from the one-jet, two-jet and three-jet NLO calculations, respectively). Unless stated otherwise, the jet multiplicity is exclusive, labelled by 'excl', i.e. a veto on 

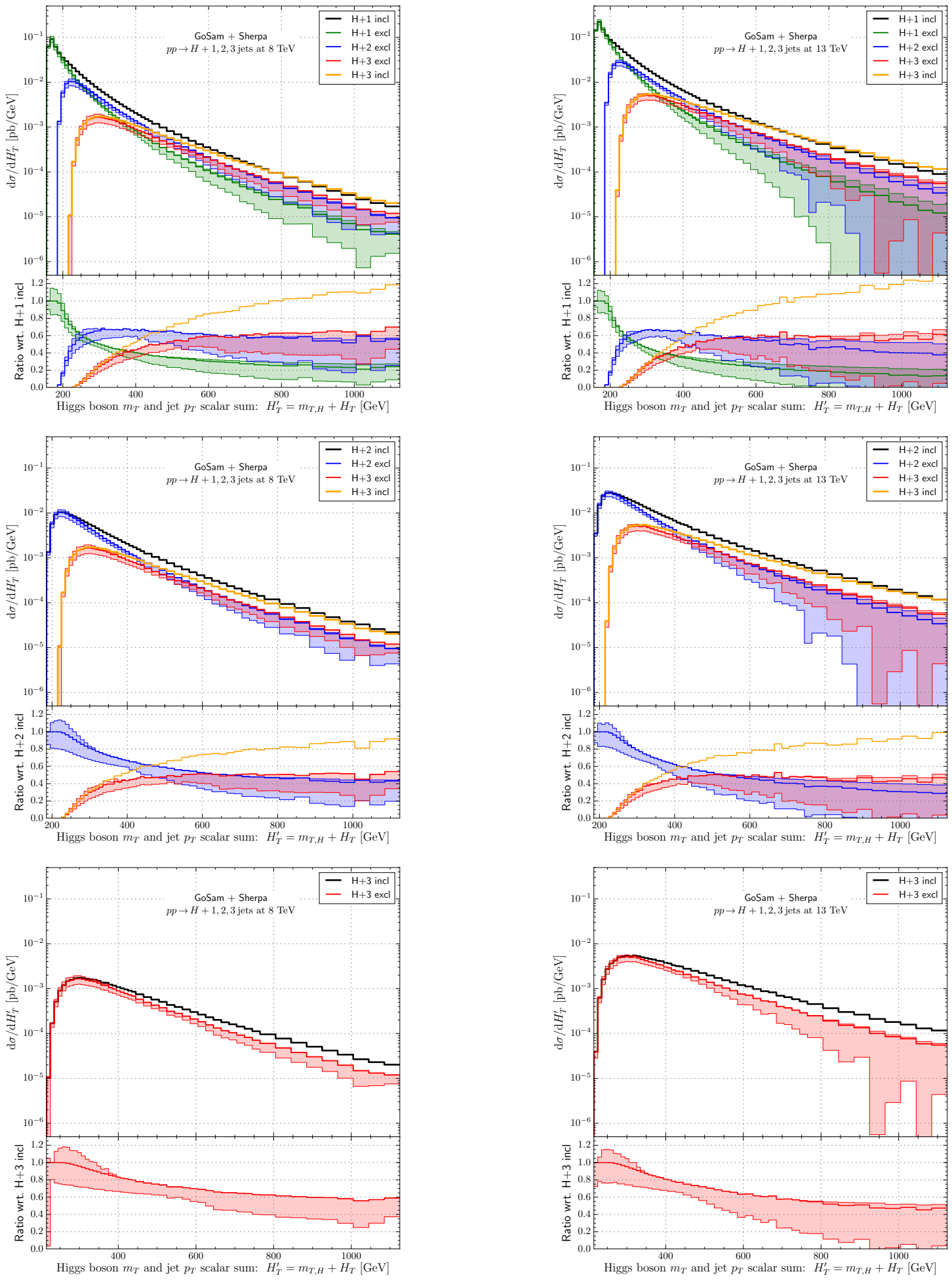

Figure 13. Exclusive NLO rates for different jet bins and their envelopes from scale variations for $H_{T}^{\prime}$ at $E_{\mathrm{cm}}=8 \mathrm{TeV}$ (left column) and $E_{\mathrm{cm}}=13 \mathrm{TeV}$ (right column). Inclusive NLO predictions for the lowest and highest jet bin are also shown (i.e. from top to bottom, $n_{\min }=1,2,3$ while $n_{\max } \equiv 3$ ) with the former serving as the reference curve in the respective ratio plots. See text for more details. 
any additional jet activity is in place. The 1-jet and the 3 -jet processes are shown twice, once for the exclusive case, and once for the inclusive case, labelled by 'incl'. The lower subpanels show each contribution normalized to the inclusive prediction of the core process, i.e. the most inclusive one, here given by the $\mathrm{H}+1$ jet process. The plots in the middle and lower panel are constructed following the same principle but using the NLO core process of increased jet multiplicity, namely $\mathrm{H}+2$ jets and $\mathrm{H}+3$ jets, respectively. The middle row of figure 13 hence depicts the same situation but without accounting for the $\mathrm{H}+1$ jet process; and, for the lower row, there are only two distributions left to show, the ones for the exclusive as well as the inclusive $\mathrm{H}+3$ jets process.

In the following, we will only discuss the results for $13 \mathrm{TeV}$. Again, finite top-quark mass corrections in principle have a strong impact on transverse momentum observables. However, at least for the transverse momentum of the Higgs boson, they were shown to be independent of the jet multiplicity [125]. Thus, the following observations are expected to also hold once $m_{t}$ effects are included.

The exclusive $\mathrm{H}+1$ jet contribution dominates below $200 \mathrm{GeV}$, but it falls off steeply towards higher values of $H_{T}^{\prime}$. The exclusive $\mathrm{H}+2$ jets contribution is negligible in the low $H_{T}^{\prime}$ region, but just above $250 \mathrm{GeV}$ it takes over from the $\mathrm{H}+1$ jet contribution and dominates the $H_{T}^{\prime}$ spectrum until the exclusive $\mathrm{H}+3$ jets contribution clearly becomes the leading one, which happens around $800 \mathrm{GeV}$. Being completely negligible up to around $250 \mathrm{GeV}$, the relative importance of the exclusive $\mathrm{H}+3$ jets contribution rises quickly surpassing the exclusive $\mathrm{H}+1$ jet curve at around $350 \mathrm{GeV}$. In the range of $500-600 \mathrm{GeV}$, it then becomes as equally important as the exclusive $\mathrm{H}+2$ jets contribution. We emphasize again that the different exclusive contributions are taken from different NLO computations, more precisely the exclusive $\mathrm{H}+n$ jets prediction has been obtained from the NLO calculation for $\mathrm{H}+n$ jets production. The inclusive $\mathrm{H}+1$ jet prediction at NLO (black line) is only used as the reference curve to allow for a better comparison between the exclusive distributions. It will by no means succeed to give a reasonable description of the data above $300 \mathrm{GeV}$ for the same reasons as outlined above. For larger values of $H_{T}^{\prime}$, its associated exclusive $\mathrm{H}+1$ jet contribution (green line) makes only about $20 \%$ of the inclusive result. A large fraction of the events (namely those with two and more jets) is therefore only described with LO accuracy, in a region where exclusive $\mathrm{H}+2$ jets and $\mathrm{H}+3$ jets events alone give rise to $40 \%$ and $60 \%$ of the inclusive $\mathrm{H}+1$ jet result, respectively. Comparing moreover the exclusive $\mathrm{H}+3$ jets (red line) to the inclusive $\mathrm{H}+$ 3 jets (orange line) result, we observe that even the fourth jet plays a very important role. Above $300 \mathrm{GeV}$ the inclusive $\mathrm{H}+3$ jets result is the second largest contribution to the $H_{T}^{\prime}$ spectrum, and it rises to $80 \%$ of the inclusive $\mathrm{H}+1$ jet result at around $500 \mathrm{GeV}$.

Based on the same philosophy, we have generated the plots where the jet multiplicity of the core process has been incremented twice. This guarantees a direct comparison between successive jet bins. The results in the middle $\left(n_{\min }=2\right)$ and bottom $\left(n_{\min }=3\right)$ panel of figure 13 convey a clear message: the pattern between the different contributions remains the same yet the magnitude of the effects decreases with an increasing number of jets in the core process. For example, focusing on a single column in the figure, there are three differential ratios, which one obtains from dividing the exclusive by the inclusive 

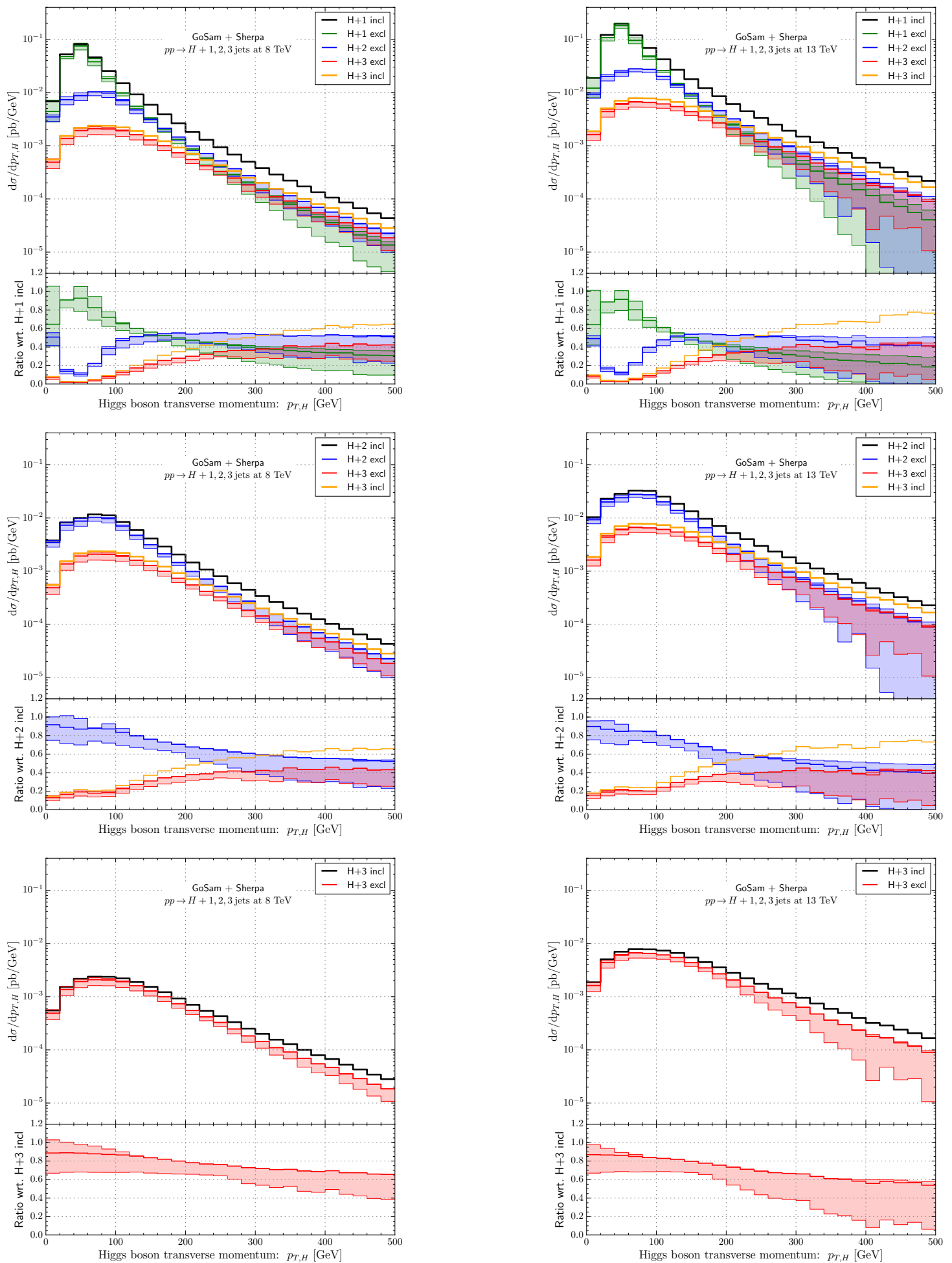

Figure 14. Exclusive NLO rates for different jet bins and their envelopes from scale variations for the $p_{T, \mathrm{H}}$ spectrum at $E_{\mathrm{cm}}=8 \mathrm{TeV}$ (left column) and $E_{\mathrm{cm}}=13 \mathrm{TeV}$ (right column). Inclusive NLO predictions for the lowest and highest jet bin are also shown (i.e. from top to bottom, $n_{\min }=1,2,3$ while $n_{\max } \equiv 3$ ) with the former serving as the reference curve in the respective ratio plots. See text for more details. 
contribution for the various $\mathrm{H}+n_{\text {min }}$ jets processes. For the final states with more jets, we then notice the rising impact of the fraction of exclusive events within the inclusive sample. The overall behavior of the three ratio curves however is very similar to the $\mathrm{H}+1$ jet case in the upper row. The exclusive $\mathrm{H}+n_{\min }$ jets contribution dominates the low $H_{T}^{\prime}$ region, but drops fast with increasing $H_{T}^{\prime}$ values to become gradually more unimportant in the high- $H_{T}^{\prime}$ range. At least one order of magnitude below the peak of the corresponding inclusive prediction, there will be a transition region where the exclusive contribution of the next jet bin, i.e. the one related to $\mathrm{H}+\left(n_{\min }+1\right)$ jets, will become similar in size. This has to be kept in mind, particularly for VBF searches at $E_{\mathrm{cm}}=13 \mathrm{TeV}$. Based on $n_{\text {min }}=2$, the $H_{T}^{\prime}$ range of the transition occurs around $500 \mathrm{GeV}$.

In conclusion, the processes of higher jet multiplicity give rise to important contributions to the $H_{T}^{\prime}$ spectrum. This does not only happen in the high- $H_{T}^{\prime}$ region but already at values of around $250 \mathrm{GeV}$. Our observations have clarified that a single $\mathrm{H}+$ jet computation at NLO falls short of giving a sufficient description of the full $H_{T}^{\prime}$ spectrum. The consistent inclusion of higher multiplicity contributions is therefore important for a reliable prediction of the distribution.

From the definition of the observable, $H_{T}^{\prime}$ is expected to be largely influenced by additional radiation, and this has been shown above. What is more striking though, is that more inclusive observables, like the Higgs boson transverse momentum, are also susceptible to the same effect. This is exemplified in figure 14. We observe that the higher multiplicity processes play an equally important role for the Higgs boson $p_{T}$ spectrum as they do for the visible energy.

Similar conclusions can be drawn from an analysis of ratios taken between successive $n$-jet cross sections. As before, it is more meaningful to look at differential distributions, but more than sufficient to just use the inclusive predictions. In our first comparison, we concentrate on the observables from above, the $p_{T}$ of the Higgs boson and the $H_{T}^{\prime}$ of the entire 'jetty' event. The corresponding distributions are shown in figure 15 where the left and right panels depict the results for $8 \mathrm{TeV}$ and $13 \mathrm{TeV}$, respectively. For better visibility, the $\mathrm{H}+1$ jet contributions have been multiplied by a factor of 10 , while the $\mathrm{H}+2$ jets results have been multiplied by a factor of 2 . The ratio plots however use the direct predictions, and thus visualize the different $R_{2 / 1}$ and $R_{3 / 2}$, i.e. the behavior of the $(n+1)$-jet cross section normalized to the $n$-jet cross section. Presented this way, the relative importance of the numerator contribution (the less inclusive case) can be better judged with respect to the contribution in which the jet multiplicity is one unit lower (the more inclusive case).

Focusing on $p_{T, \mathrm{H}}$, we observe that apart from the peak region both $R_{n+1 / n}$ quantities easily exceed $40 \%$ for the most part of the $p_{T}$ range. In the high- $p_{T}$ tail, the $\mathrm{H}+2$ jets contribution even grows as large as the $\mathrm{H}+1$ jet contribution while the $\mathrm{H}+3$ jets contribution still reaches about $60 \%$ of the $\mathrm{H}+2$ jets contribution. At $13 \mathrm{TeV}$, the $R$ values get further enhanced, notably in the far tail, as a result of the increased jet phase space. Clearly, these $R$ numbers are far from what one considers a small correction to the more inclusive spectrum. As before this is to be interpreted as an insufficient description of the all-inclusive $p_{T, \mathrm{H}}$ spectrum calling for jet-bin merging strategies. Being able to go up to $\mathrm{H}+$ 3 jets in our analysis, we still have not entered the region where the jet effects are saturating, at least not for the $p_{T}$ spectrum of the Higgs boson, as well as more severe observables. 

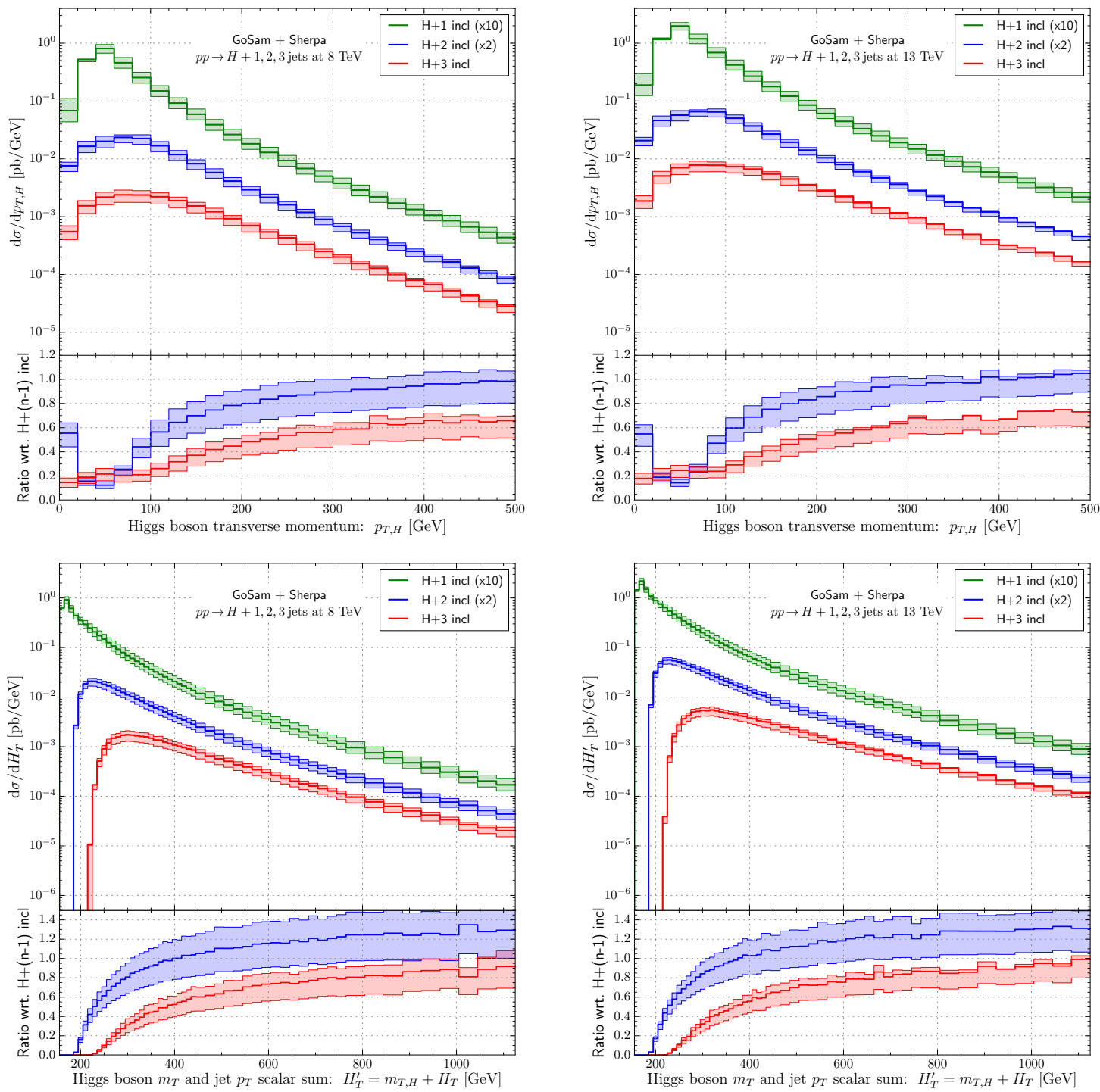

Figure 15. Successive ratios (subpanels) of inclusive $\mathrm{H}+n$ jets differential cross sections (main panels) at NLO regarding the Higgs boson's transverse momentum distribution (upper row) and the $H_{T}^{\prime}$ distribution (lower row). Results are shown for $E_{\mathrm{cm}}=8 \mathrm{TeV}$ (left column) and $E_{\mathrm{cm}}=13 \mathrm{TeV}$ (right column); rescaling factors have only been applied to the predictions of the main panels.

Qualitatively, we thus have found the same behavior as demonstrated by figures 13 and 14. The sole difference is that the effects of higher jet multiplicities are more pronounced as we consider the case of inclusive cross sections. Accordingly, for the $H_{T}^{\prime}$ observable shown in the lower row of figure 15, the effects are amplified but the overall picture remains the same. The $\mathrm{H}+2$ jets contribution supersedes the one given by $\mathrm{H}+1$ jet already at moderate values of $H_{T}^{\prime}$ for both center-of-mass energies of 8 and $13 \mathrm{TeV}$, and the $\mathrm{H}+3$ jets contribution easily makes up $60-80 \%$ of the $\mathrm{H}+2$ jets result.

Considering the transverse momentum distribution of the leading jet (at $13 \mathrm{TeV}$ ), presented in the left panel of figure 16, the same behavior is found as discussed in the 

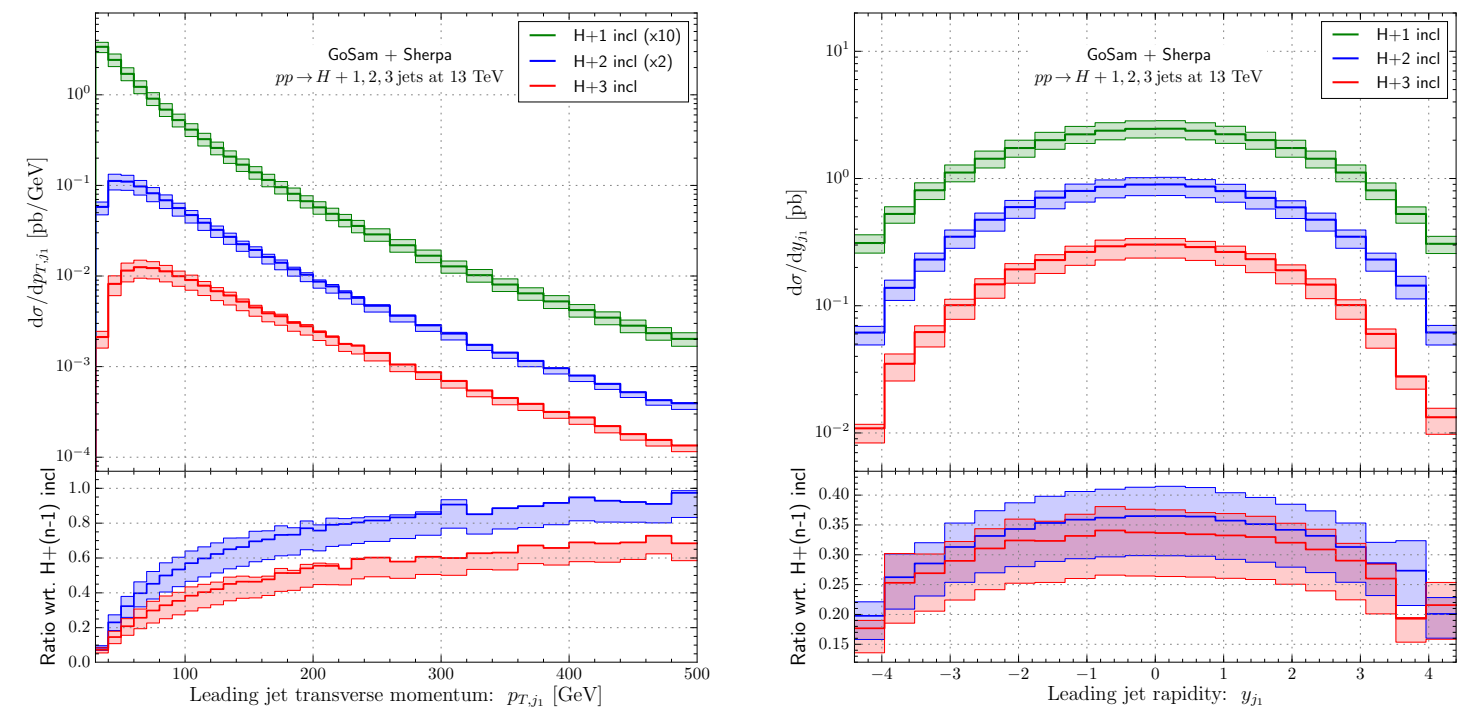

Figure 16. Successive ratios (subpanels) of inclusive $\mathrm{H}+n$ jets differential cross sections (main panels) at NLO regarding the transverse momentum distribution (left) and the rapidity distribution (right) of the leading $p_{T}$ jet using $E_{\mathrm{cm}}=13 \mathrm{TeV}$. Rescaling factors have only been applied to the predictions of the main panel shown to the left.

previous plots. It can be compared to the transverse momentum of the Higgs boson, as the hardest jet often recoils mainly against the Higgs boson. However for the rapidity of the leading jet, shown in the right panel of figure 16, the $R$ values are significantly more moderate and uniform in size exemplifying the situation for observables much less affected by additional QCD radiation.

\subsection{Comparing tagging jet selections and testing high-energy effects}

Typically the definition of tagging jets is based on the jet transverse momentum. An alternative that is more suitable for the VBF Higgs analysis, and thus worthwhile to be investigated as well, is based on a change in the jet ordering parameter. The jets in an event can be ordered according to their rapidity where the most forward and the most backward jet can be selected to form the tagging jet system. We will denote this option as $y$-tagging and the former, the standard one, as $p_{T}$-tagging. The $y$-tagging approach is theoretically motivated not only because of Higgs boson coupling measurements in the VBF channel, but also because it allows to confirm the universal properties of QCD in the high-energy limit. In this limit, $t$-channel gluon exchange dominates the cross section. Jet production can then be described by Lipatov effective vertices that are resummed in the BFKL equation [128-130]. Event generators based on a Monte Carlo solution to this equation [131-136] were constructed for the LHC in order to describe the relevant event topologies at high precision [78, 79, 137-139]. It is interesting to test how much phase space can be covered by a calculation performed in collinear factorization before highenergy resummation becomes relevant. Our calculation allows to study this question in Higgs boson production through gluon fusion for the very first time. 

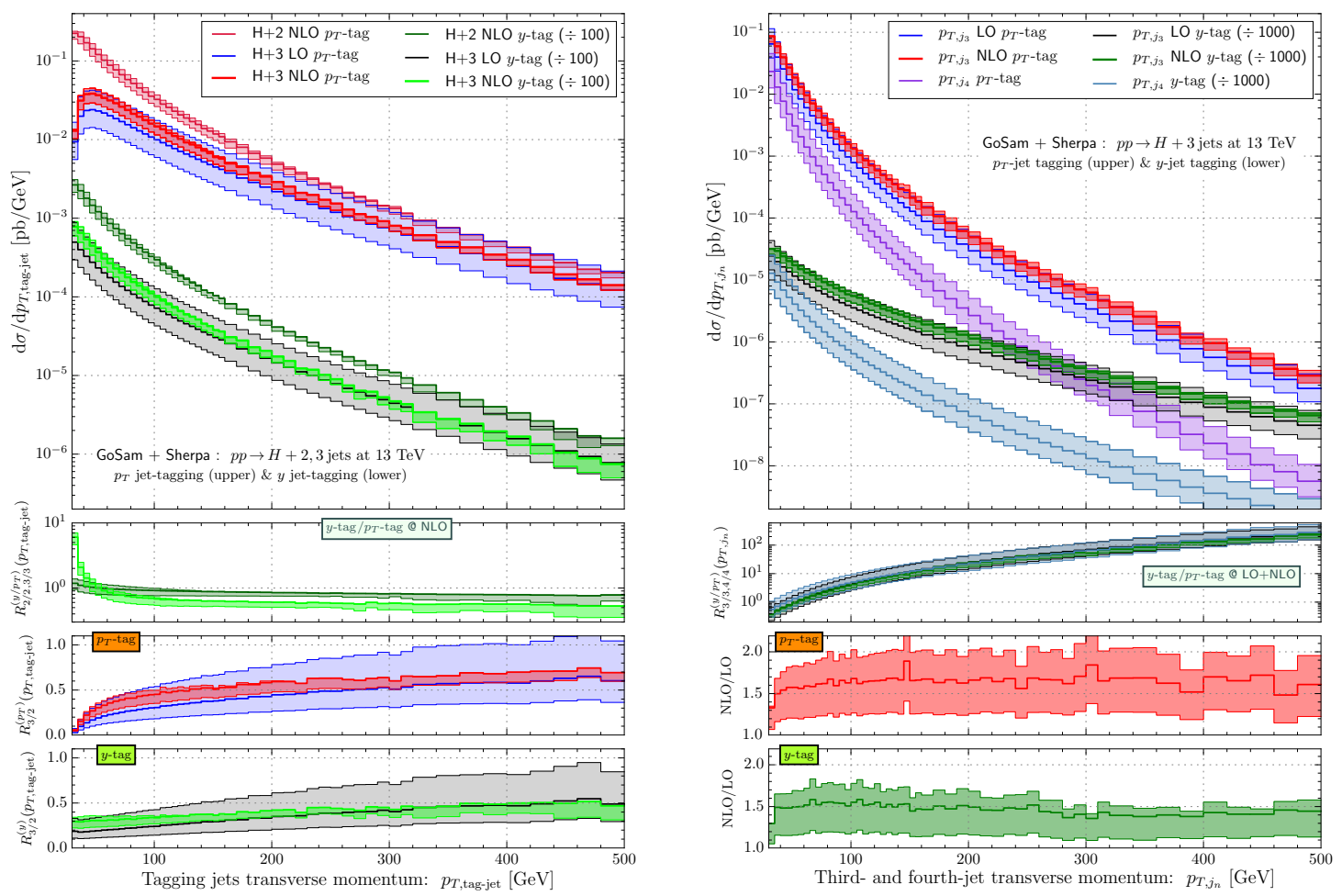

Figure 17. Transverse momentum distribution of the tagging jets (left) and the subleading jets (right) in $\mathrm{H}+2$ jets and $\mathrm{H}+3$ jets production at the $\mathrm{LHC}\left(E_{\mathrm{cm}}=13 \mathrm{TeV}\right)$. Distributions are shown for the two tagging jet definitions, $p_{T}$ jet-tagging and $y$ jet-tagging. Based on eq. (3.3), the subpanels display differential ratios for directly comparing the tag jet options (upper ratio plot) as well as the inclusive $n$-jet predictions for $n=3$ and $n=2$ in both tagging schemes (two lower ratio plots). Instead of $R$-quantities, the lowest subpanels on the right show $K$-factors regarding the $p_{T, j_{3}}$ spectra for both the $p_{T}$-tag and the $y$-tag case. See text for more details, and precise ratio definitions.

Naturally, the inclusive jet observables most affected by the two tagging options above are the rapidity distance of the two tag jets and the tag jet transverse momentum, which we obtain by entering the $p_{T}$ value for each of the two tagging jets in the event. For an LHC energy of $13 \mathrm{TeV}$, the spectrum for the latter is shown in figure 17 together with the transverse momentum distribution of the subleading jets. The rapidity distance is instead shown on the left in figure 18 whereas on the right we display the $y_{\mathrm{H}, j_{1} j_{2}}^{*}$ observable used to quantify the (averaged) rapidity difference between the tag jets and the Higgs boson. Its definition is given by

$$
y_{\mathrm{H}, j_{1} j_{2}}^{*}=\left|y_{\mathrm{H}}-\left(y_{j_{1}}+y_{j_{2}}\right) / 2\right| .
$$

The two tag jet selections are labelled (in short) by $p_{T}$-tag and $y$-tag. The upper panels depict the NLO result for $\mathrm{H}+2$ jets production at the LHC as well as the LO and NLO result for the $\mathrm{H}+3$ jets case. For better readability of the plots, all $y$-tag based predictions have been rescaled by a factor of $1 / 100$, at least. The lower panels show three different types of comparisons: at the top, the NLO ratios between the $y$-tag result and the $p_{T}$-tag 
result are displayed for both the $\mathrm{H}+2$ jets (dark green) and the $\mathrm{H}+3$ jets (light green) process. The corresponding ratios are denoted by $R_{2 / 2}^{\left(y / p_{T}\right)}$ and $R_{3 / 3}^{\left(y / p_{T}\right)}$, respectively. At the bottom, we then use separate plots to present the 3 -jet versus 2 -jet differential cross section ratios for each tagging scheme: $R_{3 / 2}^{\left(p_{T}\right)}$ is shown in the middle pane while $R_{3 / 2}^{(y)}$ is shown at the very bottom of the figures. For $p_{T}$-tagged ( $y$-tagged) jets, the bands shaded in blue (grey) and red (light green) have been obtained from using different numerators, given respectively by the $\mathrm{LO}$ and $\mathrm{NLO} \mathrm{H}+3$ jets predictions (taking standard scale variations into account), but a common denominator as given by the NLO $\mathrm{H}+2$ jets prediction.

There is one exception to this plotting convention. The subleading-jets plot in the right panel of figure 17 only contains $\mathrm{H}+3$ jets predictions, namely the third-jet $p_{T}$ spectra at $\mathrm{LO}$ and NLO as well as the LO accurate $p_{T}$ distributions regarding the fourth jet. ${ }^{4}$ Accordingly, we show the tagging scheme ratios for the three types of jet $p_{T}$ predictions but replace the $R_{3 / 2}$ panels for each tag jet selection by panels presenting the respective $K$-factors for the $p_{T}$ distribution of the third jet.

Figure 17 (left) exemplifies the change in the $p_{T}$ spectra when switching between the tag jet options as well as jet bins. The $y$-selection leads to softer tag jets than the $p_{T^{-}}$ selection, which is most easily seen in the upper ratio plot. For the $\mathrm{H}+3$ jets case, the effect is much stronger as the production of a third, softer jet has to arise from the phase space between threshold and second tag jet $p_{T}$. The enforced $p_{T}$ hierarchy of the jets therefore leads to the near-threshold depletion for very soft $p_{T}$-tag jets. For both $p_{T}$ and $y$ jet-tagging, the $\mathrm{H}+3$ jets NLO results are very similar to the respective $\mathrm{LO}$ results, albeit they show a reduced scale uncertainty combined with a slight tendency of favoring lower jet $p_{T}$. Compared to the $\mathrm{H}+2$ jets NLO calculations, the shape of the tag jet distributions changes mostly in the lower- $p_{T}$ region as nicely illustrated by the $R_{3 / 2}$ panels. In the $y$ tag scheme, the change happens more gradually, particularly at NLO, while in the $p_{T}$-tag scheme it occurs fairly drastically for the reasons just given above. In both cases, the plots indicate that the emission of a third jet, especially at large $p_{T}$, is very likely. The large radiative corrections to the $\mathrm{H}+2$ jets final state are described more precisely by a $\mathrm{H}+3$ jets than by a $\mathrm{H}+2$ jets NLO calculation (i.e. essentially $\mathrm{H}+3$ jets $\mathrm{LO}$ one), leading to largely reduced scale uncertainties. Altogether, this confirms the findings of section 3.4.

It is interesting to also consider the subleading jets shown in figure 17 (right), which are defined for both selections as the jets with highest transverse momentum, excluding the tag jets. We observe that the subleading jets have very different $p_{T}$ spectra in the two selections, which is simply due to the fact that the hardest jet is preferably produced at central rapidity, revealing itself as the leading tag jet in the $p_{T}$-selection, but the first subleading jet in the $y$-selection. The differential $K$-factors shown in the middle and lower ratio plots display only modest deviations from a uniform behavior over the entire kinematic range. Recalling the 'wimpiest' jet discussion around figure 10, we are able to confirm the earlier findings. We see that they also apply to the $y$-tagging case for which we notice a further reduction of the theoretical uncertainty.

\footnotetext{
${ }^{4}$ Owing to our parameter settings, the $\mathrm{H}+2$ jets NLO scenario is represented implicitly in figure 17 through the $p_{T, j_{3}}$ spectra of the $\mathrm{H}+3$ jets computations at $\mathrm{LO}$.
} 

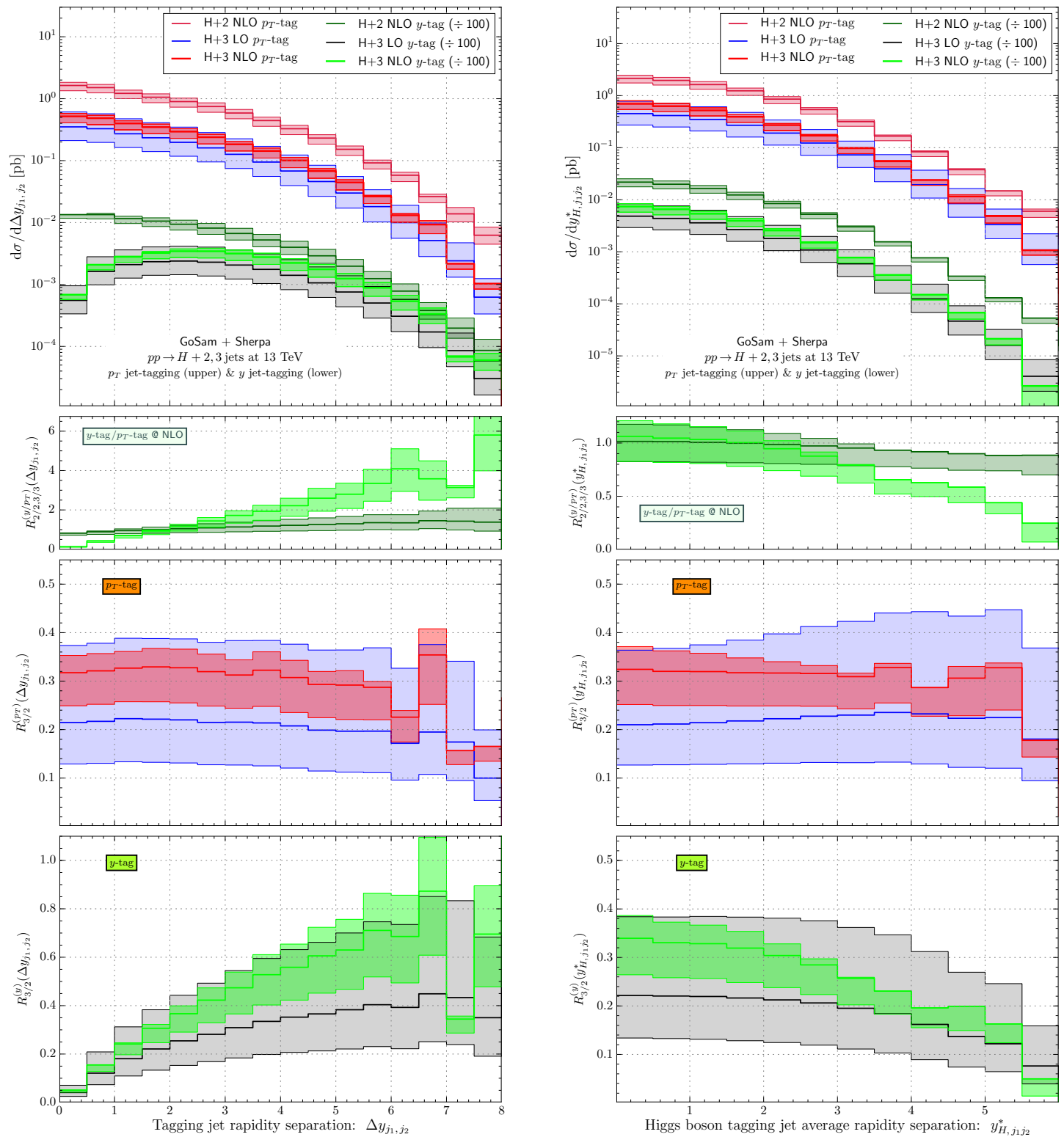

Figure 18. NLO predictions for rapidity difference observables in $\mathrm{H}+2$ jets and $\mathrm{H}+3$ jets production at the $13 \mathrm{TeV}$ LHC. The $y$ separation between the tagging jets is presented on the left while the $y^{*}$ variable measuring the distance between the Higgs boson and the two tagging jets is depicted in the right panel. Distributions are shown for the two tagging jet definitions, $p_{T}$ and $y$ jet-tagging. Using eq. (3.3), the subpanels display differential ratios for directly comparing both tag jet options with each other (upper ratio plot) as well as the inclusive $n$-jet predictions for $n=3$ and $n=2$ for each tagging scheme (two lower ratio plots). See text for more details, and precise ratio definitions. 
Relying on the $y$-tagging scheme, figure 18 (left) shows a significant shift towards larger rapidity differences, as compared to the $p_{T}$-tagging. Jets are still predominantly produced centrally, because we apply democratic transverse momentum cuts, and the phase space for centrally produced jets is larger. With an increasing number of jets, the $y$-tagging becomes more effective and different from the $p_{T}$ case. The corresponding $\mathrm{H}+3$ jets predictions deviate therefore more pronouncedly from those generated by the $p_{T}$ scheme. Focusing on the NLO results, this is nicely demonstrated by the $R^{\left(y / p_{T}\right)}$ panel to the left of figure 18 .

Another important feature appears in the lower ratio plots on the left of figures 17 and 18. On the one hand, $R_{3 / 2}^{\left(p_{T}\right)}\left(p_{T, \text { tag-jet }}\right)$ shows a roughly logarithmic rise, owing to the large radiative corrections in the $\mathrm{H}+2$ jets process. At the same time, $R_{3 / 2}^{\left(p_{T}\right)}\left(\Delta y_{j_{1}, j_{2}}\right)$ is approximately flat. On the other hand, $R_{3 / 2}^{(y)}\left(\Delta y_{j_{1}, j_{2}}\right)$ is roughly proportional to $\Delta y$ featuring an even steeper slope at NLO, indicating an increasing NLO over LO ratio. We can compare this, on a qualitative level, to the results presented in ref. [140] where $R_{3 / 2}^{(y)}\left(\Delta y_{j_{1}, j_{2}}\right)$ had also been computed. This calculation was performed in an approach based on the high-energy resummation — using HEJ [79, 138] — and compared to results from collinear factorization - using MCFM [37]. The authors observed a considerable discrepancy between the two calculations, particularly for large rapidity differences. In this context, it is important to stress that MCFM describes the $\mathrm{H}+3$ jets topologies at $\mathrm{LO}$ accuracy only. Comparing their findings with figure 18 (left), we note that the discrepancy observed in [140] is largely reduced by the NLO correction to the $\mathrm{H}+3$ jets process.

Closely related to the rapidity separation of the tag jets, figure 18 (right) presents the other important $\Delta y$ variable considered here. We see that $y_{\mathrm{H}, j_{1} j_{2}}^{*}$ behaves very similar to $\Delta y_{j_{1}, j_{2}}$ as long as one retains the $p_{T}$ jet-tagging. This carries over to the $R_{3 / 2}^{\left(p_{T}\right)}$ ratios, which again turn out to be fairly even. The other two ratio plots however reveal the differences, even though the $p_{T}$-tag and $y$-tag based $y_{\mathrm{H}, j_{1} j_{2}}^{*}$ distributions seemingly look alike in the figure's main plot. We notice that the inclusive $R^{\left(y / p_{T}\right)}$ and $R_{3 / 2}^{(y)}$ ratios show the exactly opposite behavior compared to the respective curves from the previous case. For example, the $R_{3 / 2}^{(y)}$ NLO curve as a function of the variable $y_{\mathrm{H}, j_{1} j_{2}}^{*}$ is gradually decreasing while it is approximately linearly rising as a function of the rapidity distance $\Delta y_{j_{1}, j_{2}}$. Hence, in the $y$-tag scheme, particularly for the $\mathrm{H}+3$ jets case, the tendency to emit the Higgs boson, rapidity-wise, right between the tag jets becomes slighly more pronounced.

\section{Vector boson fusion phenomenology}

The production of a Higgs boson in the VBF channel is phenomenologically highly relevant, as it allows to measure the couplings between electroweak gauge bosons and the Higgs boson. It also provides sensitivity to the $C P$-structure of the Higgs couplings [141], as well as access to possible anomalous couplings in both the Higgs sector and the electroweak sector of the Standard Model.

As gluon fusion is an irreducible background to the VBF channel, the challenging task for theory is to provide a precise prediction of its rate compared to the signal. NLO precision 

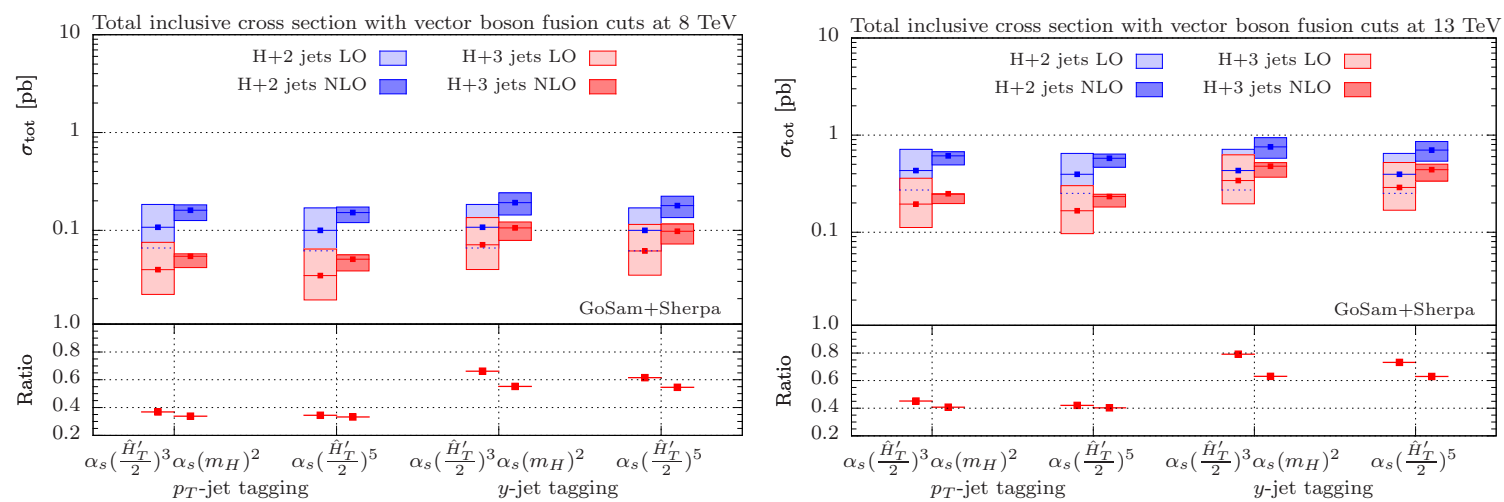

Figure 19. Total cross sections at LO (left 'towers') and NLO (right 'towers') for $\mathrm{H}+2$ jets (blue) and $\mathrm{H}+3$ jets (red) production at the LHC using VBF kinematical cuts and two different tagging jet definitions. Results are shown for the two scale choices A, cf. eq. (2.8a), and B, cf. eq. (2.8b), as well as the two LHC energies of $8 \mathrm{TeV}$ (left plot) and $13 \mathrm{TeV}$ (right plot). The lower part of each plot depicts the inclusive cross section ratios $r_{3 / 2}$ for the different scales and tag jet approaches.

for the signal (by which we mean the VBF processes with up to three jets) has already been achieved $[24,25,142,143]$. In this section, we therefore focus on the background.

The main obstacle is the extraction of the exclusive $\mathrm{H}+2$ jets cross section in the fiducial region of typical VBF analyses. We have already seen in sections 3.4 and 3.5 that higher-multiplicity final states contribute sizeably to the inclusive cross section. If we extract the effect of a central or global jet veto on the $\mathrm{H}+2$ jets final state from the NLO $\mathrm{H}+2$ jets calculation, the prediction is of leading-order accuracy and the associated theoretical uncertainty is therefore large. A more reliable fixed-order prediction is derived from a simultaneous calculation of $\mathrm{H}+2$ jets and $\mathrm{H}+3$ jets. In this case, one obtains the exclusive $\mathrm{H}+2$ jets rate as the difference between the inclusive $\mathrm{H}+2$ jets result and the inclusive $\mathrm{H}+3$ jets result in the vetoed region of the phase space, thus improving on the theoretical accuracy of logarithmically enhanced contributions, which are related to the veto on additional jet activity [144]. In addition, the kinematic distribution of $\mathrm{H}+3$ jets events may also help to devise phase space constraints for improving the purity of an LHC event sample. In this section, we therefore provide results for the gluon fusion process when applying the typical VBF selection criteria as described in eq. (2.6),

$$
m_{j_{1} j_{2}}>400 \mathrm{GeV}, \quad\left|\Delta y_{j_{1}, j_{2}}\right|>2.8,
$$

and we focus in particular on observables where we expect different shapes between the VBF signal and the gluon fusion background.

\subsection{Cross sections and scale dependence}

We start our discussion with the total cross sections as displayed in figure 19. In contrast to section 3.1 where we implemented more generic multijet cuts, we refrain here from including the $\mathrm{H}+1$ jet result, as the VBF signal requires at least two jets. Having excluded the fixed scale as a sensible choice in the sections above, we only show the two scale choices $\mathrm{A}$ and $\mathrm{B}$ for comparison. At the same time, we however include two different definitions 

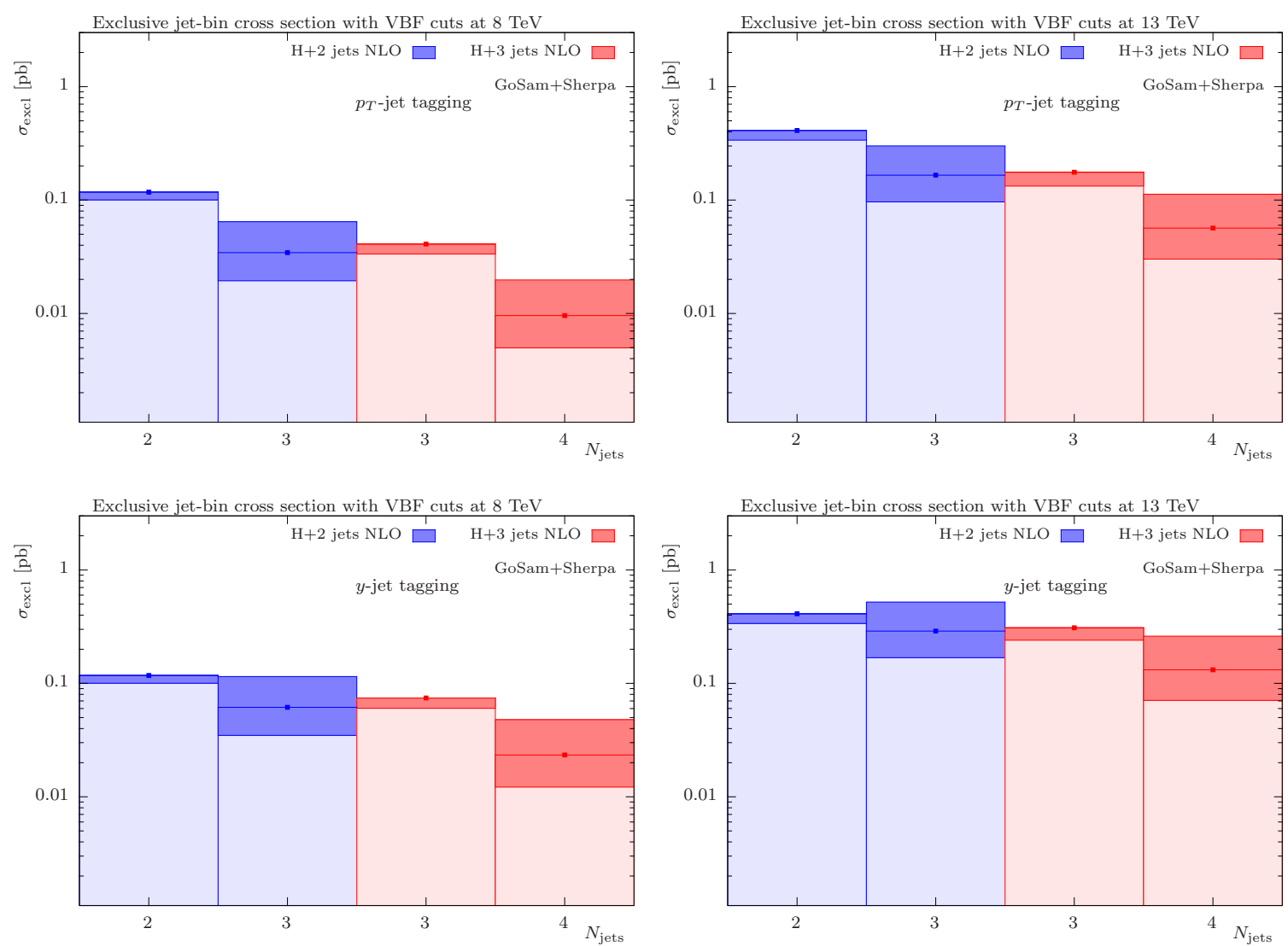

Figure 20. Exclusive jet cross sections in $\mathrm{H}+2$ jets and $\mathrm{H}+3$ jets production at the $8 \mathrm{TeV}$ (left) and $13 \mathrm{TeV}$ (right) LHC after application of typical VBF selection constraints (using scale choice $\mathrm{B}$, cf. eq. (2.8b), and jet separation parameter $R=0.4$ ). The upper and lower set of plots display the results based on different jet-tagging strategies, namely for $p_{T}$ jet-tagging and $y$ jet-tagging, respectively. Note that the figure layout corresponds to that of figure 4 .

to identify the tagging jets: we define them $(i)$ as the two jets with the largest transverse momenta, (again) referred to in the following as ' $p_{T}$-tag', and (ii) as the pair which spans the largest rapidity interval between them, (again) referred to as ' $y$-tag'. The third and fourth jet are then those among the remaining jets with the largest and second largest transverse momentum, respectively.

Figure 19 contains several different messages. We notice that in all cases, the two scale choices A and B give rather similar results. Furthermore, the changes to the plot pattern are marginal when one increases the center-of-mass energy from $8 \mathrm{TeV}$ to $13 \mathrm{TeV}$. The interesting comparison finally is between the results of the two tag jet approaches. We observe that the choice of the tagging jet scheme has a considerable impact on the total cross section for the $\mathrm{H}+3$ jets process whereas for the $\mathrm{H}+2$ jets process, the cross section is almost unaffected by it. This is easily understood as the latter case is independent of the tagging scheme at leading order. At NLO, a mild dependence is then introduced yielding enhanced $y$-tag cross sections by about $20 \%$. This effect gets further enhanced for the $\mathrm{H}+3$ jets process, leading to a difference in the total cross section of almost a factor of two $(\sim 80 \%$ at LO 
and $\sim 90 \%$ at NLO). This shows that the $y$-tag definition is much more sensitive to additional radiation beyond the two tagging jets. Thus, employing $y$-tagging we also find larger $K$-factors, of about $20 \%$ for the $\mathrm{H}+2$ jets and $10 \%$ for the $\mathrm{H}+3$ jets process. As before the lower panels have been reserved to show successive jet bin ratios, i.e. here it is meaningful to compare the various results for $r_{3 / 2}$. In the $y$-tag approach they happen to be increased for obvious reasons; in particular, the LO ratios are larger than their NLO counterparts owing to the zero effect from $y$-tagging on the $\mathrm{H}+2$ jets denominator at LO. However, independent of both the choice of the tagging scheme and the collider energy, we see good agreement between the LO and NLO ratios. Similarly, the effect of the scale choice is almost negligible.

As before, a first assessment of the impact of higher-jet multiplicities can be achieved by exhibiting the anatomy of the $\mathrm{H}+n$ jets NLO cross sections in terms of distinct jet bin contributions. For the $\mathrm{H}+2$ jets and the $\mathrm{H}+3$ jets process, figure 20 shows how the inclusive predictions divide up into exclusive jet cross sections based on the two tagging jet definitions and at both center-of-mass energies considered in this paper. The $\mathrm{H}+2$ jets NLO calculation accordingly contributes to the two-jet and three-jet bins while the $\mathrm{H}+3$ jets NLO calculation contributes to the three-jet and four-jet bins where we have to keep in mind that the respective $(n+1)$-jet contributions are only described with leading order accuracy. Comparing these to the results presented in figure 4 where no topological cuts were applied, it can be observed that there is a relative enhancement of the respective $(n+1)$-jet contributions in the VBF fiducial region. For the $y$-tag scheme, this effect is somewhat stronger than for the $p_{T}$-tag scheme. The same is true comparing the $13 \mathrm{TeV}$ results to the $8 \mathrm{TeV}$ ones. This relative enhancement of the $(n+1)$-jet contributions implies that the portion of the cross section, which is only described at leading-order accuracy, becomes more important. Turning this argument around, this means that the theoretical uncertainty is increased in the VBF fiducial region as the leading-order pieces of the calculation have a larger contribution to the total cross section than in the simple dijet region discussed in the previous sections. This stresses the importance of the $\mathrm{H}+3$ jets calculation particularly in the context of VBF analyses as it allows to determine the radiation of a third jet with NLO accuracy.

Following the layout of table 2, we again present a more detailed summary of the total cross sections of the various calculations for our default scale and $R$-parameter choice. Besides the cross sections, table 3 lists the associated scale uncertainties, cross section ratios and jet fractions for the LHC energies of $8 \mathrm{TeV}$ and $13 \mathrm{TeV}$. The values for both tagging schemes are given separately which also applies to the $K$-factors shown in the table. Having tables 2 and 3 at hand, we are in the position to quantify the total cut efficiency associated with the VBF selection employed here. We find values of the order of $15 \%$. The LO results are always slightly above the NLO efficiencies, and we also notice an increase for larger jet multiplicity as well as collider energy. For the $\mathrm{H}+2$ jets and $\mathrm{H}+3$ jets processes using $E_{\mathrm{cm}}=8 \mathrm{TeV}$, we respectively obtain $10.2 \%$ and $12.7 \%$ at $\mathrm{NLO}(10.7 \%$ and $13.5 \%$ at LO) while we find $12.8 \%$ and $16.2 \%$ at $\mathrm{NLO}(13.4 \%$ and $17.1 \%$ at $\mathrm{LO})$ for $E_{\mathrm{cm}}=13 \mathrm{TeV}$. In addition, it is interesting to compare the cross section estimates for the exclusive $\mathrm{H}+2$ jets final states, which we obtain for both tagging schemes from the differences in the inclusive two-jet and inclusive three-jet cross sections at NLO. With respect to the $p_{T}$-tagging scheme, the $y$-tagging scheme leads to a reduction of the gluon fusion background by $20 \%$ 


\begin{tabular}{|c|c|c|c|c|c|c|c|}
\hline \multirow{2}{*}{\multicolumn{2}{|c|}{ Sample }} & \multicolumn{6}{|c|}{ Cross sections in pb for Higgs boson production with } \\
\hline & & $\geq 2$ jets & $f_{3}$ & $\geq 3$ jets & $f_{4}$ & $\geq 4$ jets & $r_{n+1 / n}$ \\
\hline Scheme & $\mathrm{H}+n$ jets & \multicolumn{3}{|c|}{$8 \mathrm{TeV} \mathrm{LHC}$} & \multicolumn{3}{|c|}{ @ LO (using NLO PDFs) } \\
\hline \multirow[t]{2}{*}{$p_{T}$-tag } & $\mathrm{H}+2$ jets & $0.100_{-38 \%}^{+69 \%}$ & 0.0 & & & & 0.344 \\
\hline & $\mathrm{H}+3$ jets & & & $0.034_{-43 \%}^{+87 \%}$ & 0.0 & & \\
\hline \multirow[t]{3}{*}{$y$-tag } & $\mathrm{H}+2$ jets & $0.100{ }_{-38 \%}^{+69 \%}$ & 0.0 & & & & 0.615 \\
\hline & $\mathrm{H}+3$ jets & & & $0.061_{-43 \%}^{+86 \%}$ & 0.0 & & \\
\hline & & & & $8 \mathrm{TeV} \mathrm{LHC}$ & & & @ NLO \\
\hline \multirow[t]{2}{*}{$p_{T}$-tag } & $\mathrm{H}+2$ jets & $0.152_{-21 \%}^{+14 \%}$ & 0.226 & $0.034_{-44 \%}^{+87 \%}$ & & & 0.333 \\
\hline & $\mathrm{H}+3$ jets & & & $0.051_{-24 \%}^{+11 \%}$ & 0.190 & $0.010_{-48 \%}^{+105 \%}$ & $(0.190)$ \\
\hline \multirow[t]{2}{*}{$y$-tag } & $\mathrm{H}+2$ jets & $0.179_{-25 \%}^{+25 \%}$ & 0.343 & $0.062_{-43 \%}^{+87 \%}$ & & & 0.546 \\
\hline & $\mathrm{H}+3$ jets & & & $0.098_{-25 \%}^{+18 \%}$ & 0.239 & $0.023_{-48 \%}^{+105 \%}$ & $(0.239)$ \\
\hline \multicolumn{2}{|l|}{$p_{T^{-}} \mid y$-tag } & $1.52 \mid 1.79$ & & $1.47 \mid 1.59$ & & & \\
\hline Scheme & $\mathrm{H}+n$ jets & & \multicolumn{2}{|r|}{$13 \mathrm{TeV}$ LHC } & \multicolumn{3}{|c|}{ @ LO (using NLO PDFs) } \\
\hline \multirow[t]{2}{*}{$p_{T}$-tag } & $\mathrm{H}+2$ jets & $0.395_{-36 \%}^{+64 \%}$ & 0.0 & & & & 0.421 \\
\hline & $\mathrm{H}+3$ jets & & & $0.166_{-41 \%}^{+81 \%}$ & 0.0 & & \\
\hline \multirow[t]{3}{*}{$y$-tag } & $\mathrm{H}+2$ jets & $0.395_{-36 \%}^{+64 \%}$ & 0.0 & & & & 0.732 \\
\hline & $\mathrm{H}+3$ jets & & & $0.289_{-41 \%}^{+81 \%}$ & 0.0 & & \\
\hline & & & & $3 \mathrm{TeV} \mathrm{LHC}$ & & & @ NLO \\
\hline \multirow[t]{2}{*}{$p_{T^{-} \text {tag }}$} & $\mathrm{H}+2$ jets & $0.577_{-19 \%}^{+11 \%}$ & 0.288 & $0.166_{-42 \%}^{+81 \%}$ & & & 0.403 \\
\hline & $\mathrm{H}+3$ jets & & & $0.233_{-22 \%}^{+6 \%}$ & 0.243 & $0.057_{-47 \%}^{+99 \%}$ & $(0.243)$ \\
\hline \multirow[t]{2}{*}{$y$-tag } & $\mathrm{H}+2$ jets & $0.700_{-23 \%}^{+23 \%}$ & 0.412 & $0.289_{-42 \%}^{+81 \%}$ & & & 0.630 \\
\hline & $\mathrm{H}+3$ jets & & & $0.441_{-24 \%}^{+14 \%}$ & 0.299 & $0.132_{-46 \%}^{+98 \%}$ & $(0.299)$ \\
\hline$p_{T^{-}} \mid y$-tag & $\mathrm{g} \quad K_{2}, K_{3}$ & $1.46 \mid 1.77$ & & $1.40 \mid 1.53$ & & & \\
\hline
\end{tabular}

Table 3. The $8 \mathrm{TeV}$ (upper half) and $13 \mathrm{TeV}$ (lower half) LHC cross sections in pb for the various parton-level Higgs boson plus jet samples used in this study. Results are reported for the $p_{T}$ as well as the $y$ jet-tagging scheme both of which are described in the main body. The cross sections have been obtained utilizing scale choice B (i.e. all scales are evaluated at $\hat{H}_{T}^{\prime} / 2$ ) supplementing the baseline selection by the application of VBF kinematic requirements as described in the text. For each center-of-mass energy, the upper and lower parts of the subtables show the LO and NLO results, respectively, together with their uncertainties (in percent) from varying scales by factors of two, up (subscript position) and down (superscript position). The NLO-to-LO $K$-factors, $K_{n}$, for the inclusive 2 -jets $(n=2)$ and 3 -jets $(n=3)$ bin, the cross section ratios $r_{3 / 2}$ and $r_{4 / 3}$ as well as the $f_{3}$ and $f_{4}$ jet fractions are given in addition. Note that for the NLO calculations, $f_{4}$ and $r_{4 / 3}$ coincide as the predictions for $\mathrm{H}+4$ jets are only $\mathrm{LO}$ accurate. 
at $8 \mathrm{TeV}$ and $25 \%$ at $13 \mathrm{TeV}$. This makes it an appealing option in VBF analyses. However, this reduction of the background rate is accompanied by an increase of the theoretical uncertainties in the $y$-tag scheme, which can be explained by the fact that the $y$-tag scheme is sensitive to soft forward jets and therefore to the infrared region. This is not the case in the $p_{T}$-tag scheme. For a meaningful approximation of the exclusive $\mathrm{H}+2$ jets cross sections, we cannot proceed without considering the scale variation uncertainties as stated

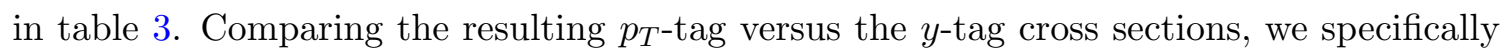
obtain $101_{-34}^{+22} \mathrm{fb}$ versus $81_{-51}^{+48} \mathrm{fb}$ and $344_{-113}^{+65} \mathrm{fb}$ versus $259_{-193}^{+173} \mathrm{fb}$ for the $8 \mathrm{TeV}$ and $13 \mathrm{TeV}$ cases, respectively. This more complete cross section analysis then puts the two schemes on a rather similar level, and, clearly, a detailed comparison of the gluon fusion background to the VBF signal is necessary to make a more precise statement about the usefulness of the $y$-tag scheme. Although its cross sections are prone to larger theory uncertainties, we may have additional handles to better control these uncertainties: we note that the scale choice $\mathrm{B}$ is seen to work well for the case of $p_{T}$ tagging; it however may not be the optimal choice for the $y$-tagging scenario. Moreover, the $y$-tag case will benefit from any given option to increase the jet $p_{T}$ threshold of VBF analyses. Raising the threshold will help improve the $y$-tag systematics in a very direct way as it reduces the dependence on the infrared region.

\subsection{Differential observables}

In order to separate events tagged by the presence of a dijet configuration which is compatible with a VBF process, experimental analyses $[7,15,145]$ rely on multivariate discriminants which are based on boosted decision trees (BDT). The typical discriminating variables used in these BDT are the invariant mass of the tagging jet system $m_{j_{1} j_{2}}$, the rapidity separation between the two tagging jets $\Delta y_{j_{1}, j_{2}}$ and their transverse momenta, $p_{T, j_{1}}$ and $p_{T, j_{2}}$. The rapidity of the leading tagging jet $y_{j_{1}}$ is also taken into account as well as the azimuthal separation and the rapidity separation between the Higgs boson and the tagging jet system, $\Delta \phi_{\mathrm{H}, j_{1} j_{2}}$ and $y_{\mathrm{H}, j_{1} j_{2}}^{*}$, respectively. Furthermore, in the measurements of the Higgs boson decaying to two photons, one also uses the transverse momentum of the diphoton system with respect to its thrust axis in the transverse plane, $p_{T, \gamma \gamma, \mathrm{t}}$, and some observables directly related to one of the two photons. The latter are not considered in the following since the Higgs boson is not decayed in our analysis. Instead of $p_{T, \gamma \gamma, \mathrm{t}}$ we will directly consider the transverse momentum $p_{T, \mathrm{H}}$ of the Higgs boson itself.

Because of the very peculiar signature of the VBF events, the tagging jet invariant mass distribution $m_{j_{1} j_{2}}$ plays a key role in determining whether an event could stem from a VBF process or not. For this reason, it is interesting to consider a third jet tagging scheme besides the $p_{T}$ jet-tagging and the $y$ jet-tagging introduced in section 3.5: one can define the two tagging jets based on the pair of jets that generates the largest invariant mass. In the presence of three or more jets, the treatment of the subleading jets is the same as in the other two schemes where they are ordered according to their transverse momenta. Although closely related to the $y$-tagging scheme, the new scheme referred to as $m_{j j}$-tagging will serve as another benchmark scenario in the following discussion.

All figures presented in this section will have the same structure: they will show our results for $\mathrm{H}+2$ jets at $\mathrm{NLO}$ and $\mathrm{H}+3$ jets at both $\mathrm{LO}$ and NLO after the application 

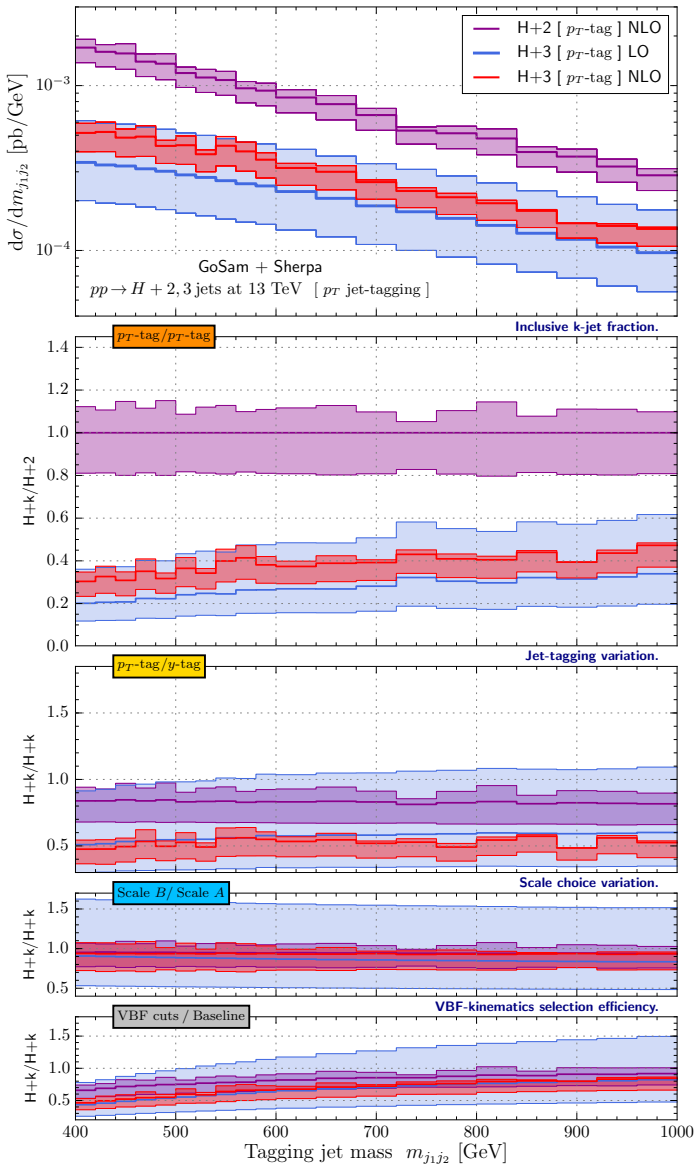
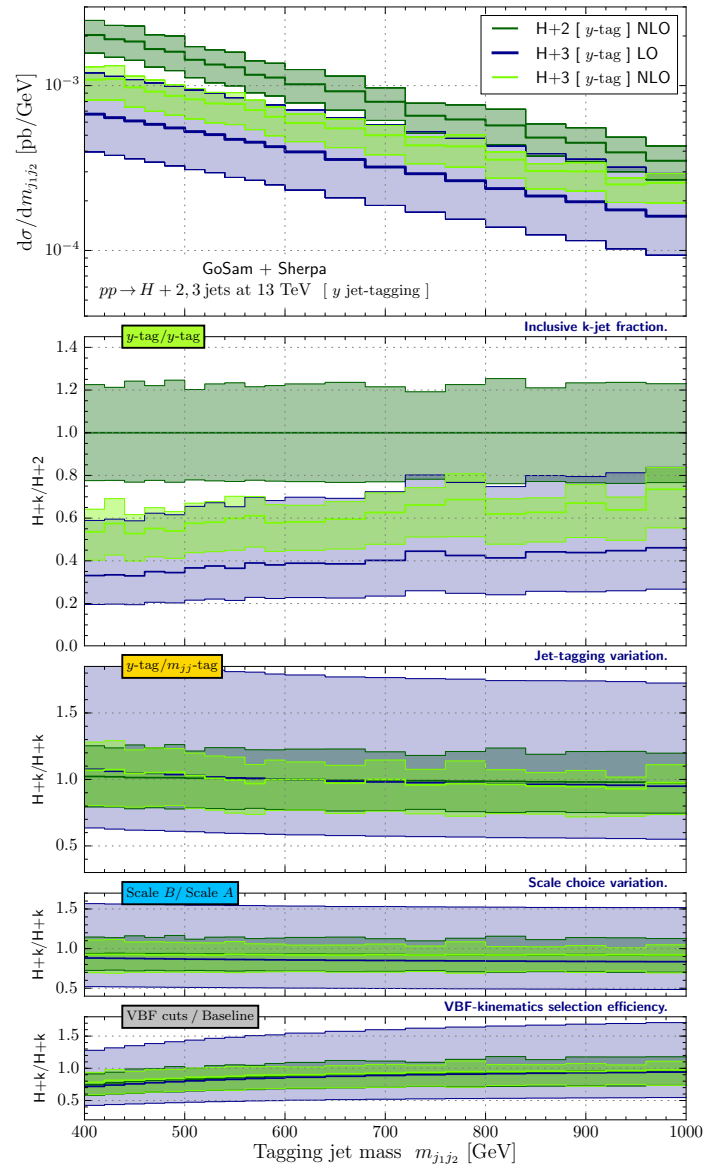

Figure 21. Impact of the $\mathrm{H}+3$ jets contribution described at NLO using scale choice B, cf. eq. (2.8b), on the tag jet invariant mass distribution, $m_{j_{1} j_{2}}$, for both tagging jet selections $p_{T}$ jet-tagging (left) and $y$ jet-tagging (right). LHC predictions at $13 \mathrm{TeV}$ center-of-mass energy are shown for the production of $\mathrm{H}+2$ jets (purple/dark green) and $\mathrm{H}+3$ jets (red/light green) at NLO as well as $\mathrm{H}+3$ jets (light blue/dark blue) at LO imposing VBF kinematic requirements in addition to the baseline selection. The four ratio plots are used to visualize, from top to bottom, the three-jet fractions, the difference between tagging schemes, the difference between various functional forms of the central scale choice and, lastly, the differential VBF cut efficiencies with respect to the dijet baseline. Scale uncertainties are indicated by the shaded bands; in the ratio plots, they are taken with respect to the central $\mathrm{H}+2$ jets NLO prediction (upper) or the respective $\mathrm{H}+n$ jets prediction for the optional tagging scheme (upper middle), the optional scale choice (lower middle) and the looser phase space constraints (lower). 
of the VBF selection criteria. The main plots on the left and on the right always contain the differential distributions, which we obtained by utilizing the $p_{T}$-tagging and $y$-tagging scheme, respectively. The differential cross sections of each main plot are accompanied by four ratio plots. Starting from the top, we display $(i)$ the three-jet fraction, $(i i)$ the ratio to an alternative tagging scheme definition $\left(p_{T}\right.$-tagging $/ y$-tagging on the left and $y$ tagging $/ m_{j j}$-tagging on the right), (iii) the ratio to a different scale choice where instead of the default scale $\mathrm{B}$, we chose scale $\mathrm{A}$ and, finally, $(i v)$ the reduction of the respective baseline cross sections due to the VBF requirements given in eq. (2.6). Note that the basic gluon fusion cuts as stated in eq. (2.5) are used to define the baseline of the respective $\mathrm{H}$ $+n$ jets analysis. In the topmost subplot, all ratios are taken with respect to the central $\mathrm{H}+2$ jets prediction at NLO accuracy using scale choice B, cf. eq. (2.8b). The other three subplots show the ratios between the respective $\mathrm{H}+2$ jets and $\mathrm{H}+3$ jets samples that were generated based on different (ii) jet tagging, (iii) scale setting and (iv) selection cut level. In all cases, the shaded bands indicate the respective standard scale uncertainties.

We start by reporting our results for the tagging jet invariant mass distribution $m_{j_{1} j_{2}}$ in figure 21. After applying the VBF cuts, the three-jet fraction varies between 0.3 and 0.4 in the $p_{T}$-tagging scheme, while it increases to $0.5-0.7$ in the $y$-tagging scheme. The contribution from $\mathrm{H}+3$ jets is therefore non-negligible, even for values of $m_{j_{1} j_{2}}$ close to the selection cut, cf. eq. (4.1). As already observed for the inclusive cross sections, when moving from $p_{T}$ to $y$ jet-tagging, the ratios between the results of different tagging strategies show a $25 \%$ increase in the cross section for $\mathrm{H}+2$ jets at NLO over the whole kinematic range and a $100 \%$ increase for $\mathrm{H}+3$ jets at both $\mathrm{LO}$ and NLO. The results are instead almost identical for $y$-tagging and $m_{j j}$-tagging. Also, varying the scale from choice B to choice A does not have a big impact, in particular at NLO. Finally, we observe that the remaining $\Delta y_{j_{1}, j_{2}}$ cut leads to a reduction of the cross section by about $50 \%$ for dijet masses around $420 \mathrm{GeV}$. Unsurprisingly, there is almost no reduction at $1 \mathrm{TeV}$, in particular in the $y$-tag scheme, where almost all dijet configurations also fulfill the rapidity separation criterion. As we will see, this change in cut efficiency can be much more dramatic for other observables, for example those where strong correlations with the selection observables are present for the bulk of the events.

We stress that the $y$-tag strategy in general yields distributions with somewhat larger uncertainty envelopes but features, at the same time, an increase in the three-jet production rate. The latter can be particularly interesting in phase space regions where the three-jet fraction gets further enhanced, providing us with additional opportunities to look for more efficient jet vetoes to reduce the gluon fusion background but keep the VBF signal. For example, in the $y$-tag scheme, one minus the three-jet rate drops to $30 \%$ in the high- $m_{j_{1} j_{2}}$ region while it remains at $60 \%$ for $p_{T}$ jet-tagging. When multiplying these numbers by the respective inclusive two-jet cross sections, one arrives at an approximation of the jet veto effect, which (expressed in terms of the $p_{T}$-tag $\mathrm{H}+2$ jets cross section) amounts to $\lesssim 40 \%$ and $\sim 60 \%$ in the $y$-tag and $p_{T}$-tag scheme, respectively. Thus, although the $p_{T}$-tagging yields smaller inclusive cross sections, the apparent advantage is eventually overcompensated by the rise of the three-jet fraction seen in the $y$-tag scheme. The goal therefore is to find an observable where this effect can be exploited in an optimal way. 

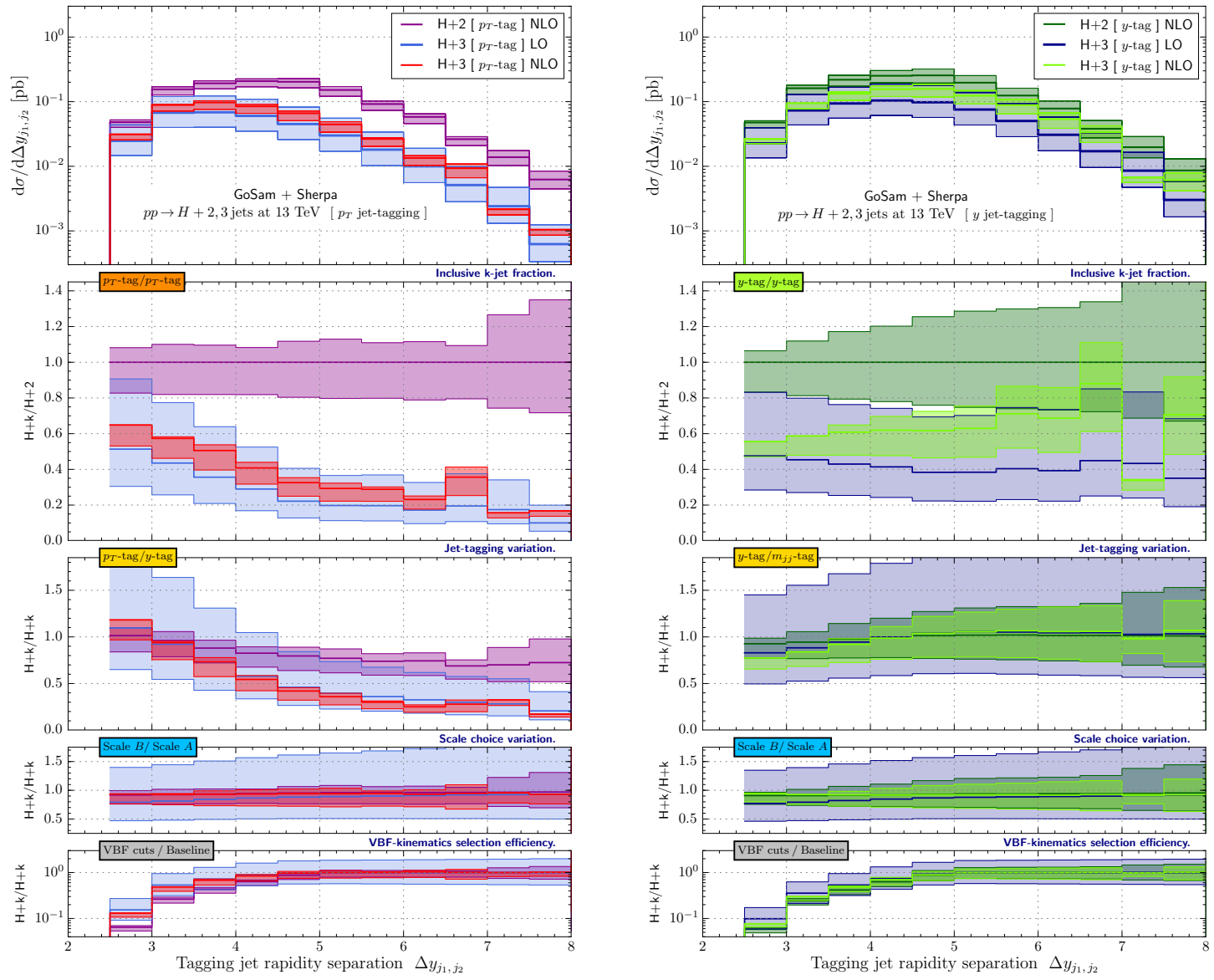

Figure 22. Impact of the $\mathrm{H}+3$ jets contribution described at NLO using scale choice B, cf. eq. (2.8b), on the tagging jet rapidity separation, $\Delta y_{j_{1}, j_{2}}$, for both tag jet selections $p_{T}$ jettagging (left) and $y$ jet-tagging (right). LHC predictions at $13 \mathrm{TeV}$ center-of-mass energy are shown for several $\mathrm{H}+n$ jets processes imposing VBF kinematic requirements in addition to the baseline selection. Note that the figure layout is the same as in figure 21 whose caption provides more details.

We continue our discussion by showing the distribution of the tagging jet rapidity separation in figure 22. This is the second observable, which one utilizes to impose the VBF topological constraints on the gluon fusion background. In the $p_{T}$-tagging scheme (left plot), we observe an important change in the shape of the $\mathrm{H}+3$ jets distribution where the peak occurs at considerably lower values of $\Delta y_{j_{1}, j_{2}}$. The presence of a further jet thus gives an additional contribution which is as large as $60 \%$ right above the cut. This decreases to less than $20 \%$ for $\Delta y_{j_{1}, j_{2}}>7$, as opposed to the cases of $y$ - and $m_{j j}$-tagging where it instead remains approximately constant. Again, as discussed in section 3.5 these findings reproduce the high-energy behavior reported in ref. [140]. Furthermore, inspecting the subpanels at the very bottom, we are led to the conclusion that events with a rapidity interval of at least five units automatically fulfill the $m_{j_{1} j_{2}}$ cut, independent of the tagging scheme. Also, varying the scale choice has almost no impact. 

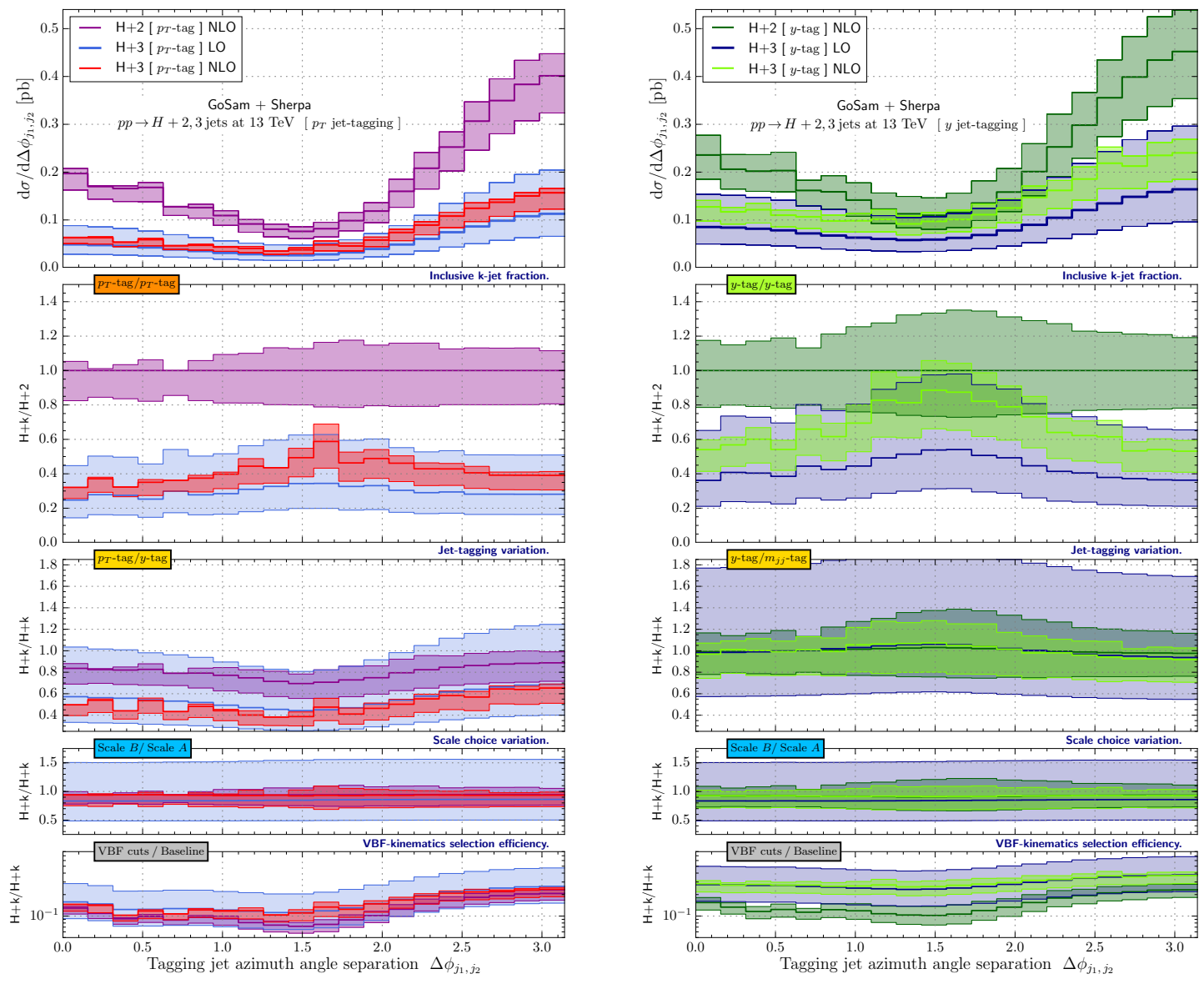

Figure 23. Impact of the $\mathrm{H}+3$ jets contribution described at NLO using scale choice B, cf. eq. (2.8b), on the tagging jet azimuthal separation, $\Delta \phi_{j_{1}, j_{2}}$, for both tag jet selections $p_{T}$ jettagging (left) and $y$ jet-tagging (right). LHC predictions at $13 \mathrm{TeV}$ center-of-mass energy are shown for several $\mathrm{H}+n$ jets processes imposing VBF kinematic requirements in addition to the baseline selection. Note that the figure layout is the same as in figure 21 whose caption provides more details.

One of the most important distributions in the VBF process is the difference in the azimuthal angle $\phi$ between the two tagging jets. It allows one to distinguish between the different possible $C P$-structures of the Higgs boson, and it is also an interesting variable to detect anomalous couplings. Here, we present the contribution from the gluon fusion channel after application of the VBF cuts; the results are shown in figure 23. Comparing the $\mathrm{H}+3$ jets $\left(p_{T}\right.$-tag) results with the ones obtained from the basic cuts, cf. figure 12, a clear change in shape, in particular for large values of $\Delta \phi_{j_{1}, j_{2}}$, is evident. The principal shape of this observable depends on the $C P$-structure of the Higgs boson-gluon coupling [146], but it becomes more pronounced when applying VBF cuts. This can be easily understood by recalling that the VBF topology requirements, namely large invariant mass and a considerable difference in rapidity, force the two tag jets into a back-to-back configuration, which is given at $\Delta \phi_{j_{1}, j_{2}} \lesssim \pi$. Moreover, the bottom ratio plots indicate that the VBF selection 

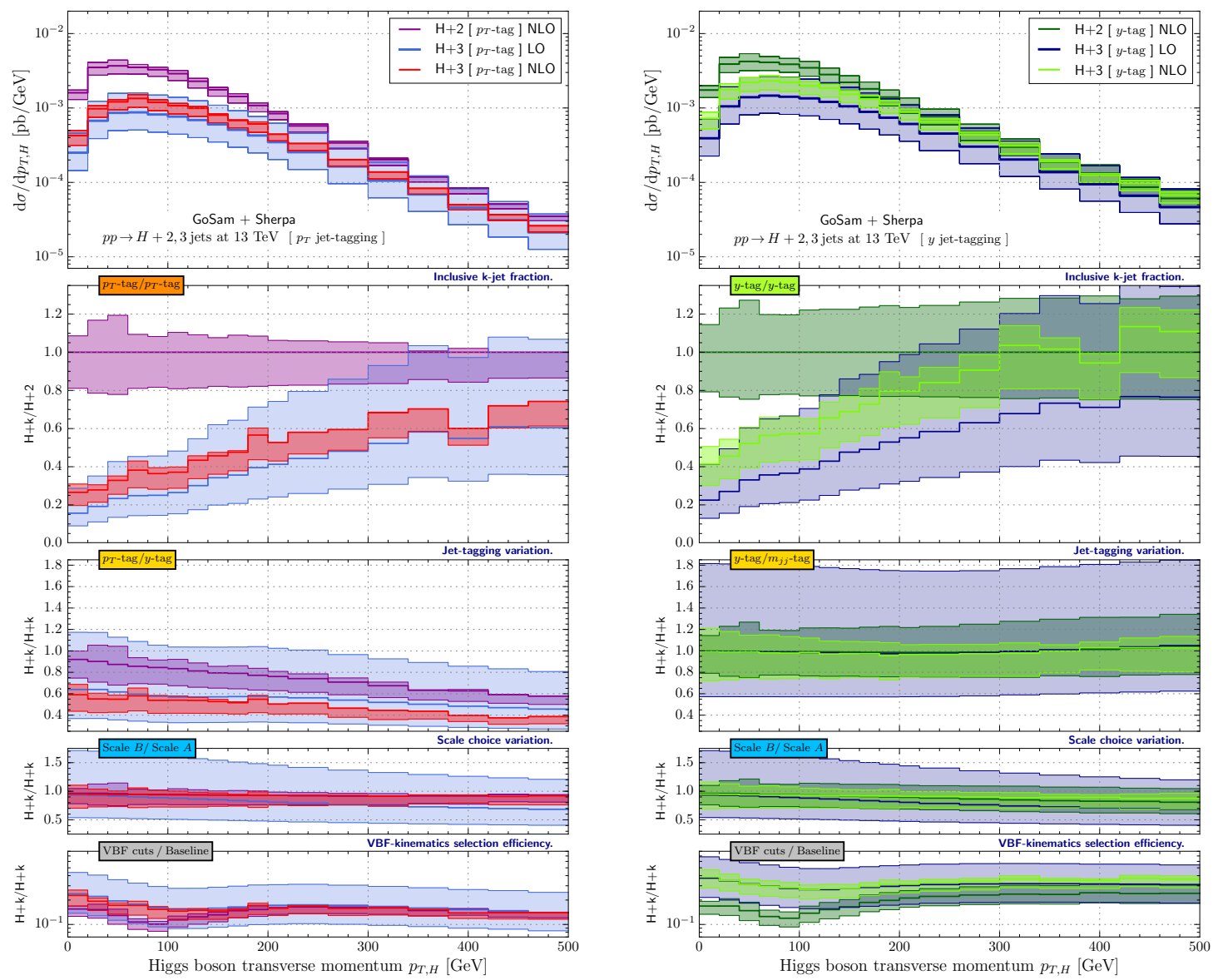

Figure 24. Impact of the $\mathrm{H}+3$ jets contribution described at NLO using scale choice B, cf. eq. (2.8b), on the Higgs boson transverse momentum, $p_{T, \mathrm{H}}$, for both tag jet selections $p_{T}$ jettagging (left) and $y$ jet-tagging (right). LHC predictions at $13 \mathrm{TeV}$ center-of-mass energy are shown for several $\mathrm{H}+n$ jets processes imposing VBF kinematic requirements in addition to the baseline selection. Note that the figure layout is the same as in figure 21 whose caption provides more details.

efficiency drops to a minimum for perpendicular tag jets where $\Delta \phi_{j_{1}, j_{2}} \approx \frac{\pi}{2}$. Interestingly, this is also where the largest scale dependence occurs, originating from the stronger influence of the three-jet configurations. For the $p_{T}$-tag case, the three-jet fraction approximately doubles in this region from 0.3 at LO to 0.6 at NLO. In the $y$-tagging scheme, as well as in the $m_{j j}$ approach, the effect is even more pronounced showing an impact of the $\mathrm{H}+3$ jets contribution that reaches almost $90 \%$ of the predicted value for $\mathrm{H}+2$ jets at NLO. Hence, with respect to the $p_{T}$ jet-tagging, the depletion near the perpendicular azimuth is less stringent and more severely affected by radiative effects.

Turning now to the transverse momentum distribution of the Higgs boson, displayed in figure 24, we observe that the shape remains largely unaffected by the application of the more stringent VBF cuts with respect to the more liberal dijet selection (cf. figure 14). This is no surprise as the additional VBF cuts do not act directly on the Higgs boson itself. 

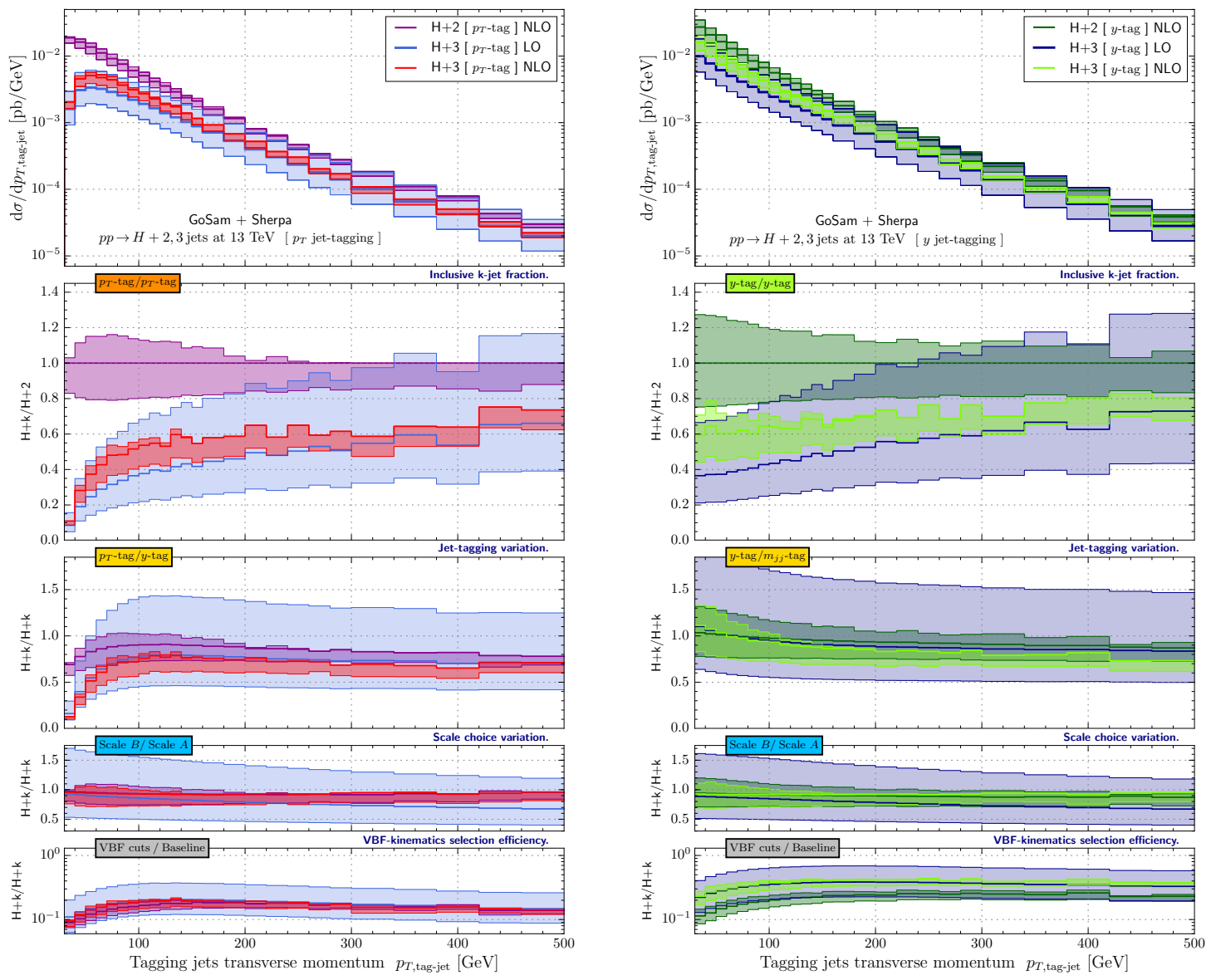

Figure 25. Impact of the $\mathrm{H}+3$ jets contribution described at NLO using scale choice B, cf. eq. (2.8b), on the transverse momentum distribution of the tagging jets, $p_{T, \text { tag-jet }}$, for both tag jet selections $p_{T}$ jet-tagging (left) and $y$ jet-tagging (right). LHC (inclusive tag jets) predictions at $13 \mathrm{TeV}$ center-of-mass energy are shown for several $\mathrm{H}+n$ jets processes imposing $\mathrm{VBF}$ kinematic requirements in addition to the baseline selection. Note that the figure layout is the same as in figure 21 whose caption provides more details.

The cross section, however, decreases by almost an order of magnitude over the whole kinematic range in the $p_{T}$-tagging scheme while the reduction again amounts to only a factor of 3 in the $y$-tagging as well as in the $m_{j j}$-tagging scheme. The latter selections also yield the harder $p_{T}$ tails as one can see from the second ratio plot in the left part of the figure. Irrespective of the employed tagging scheme, the choice for one or the other central renormalization scale at most introduces a mild tilt in the distributions. Consequently many observations made concerning figure 8 still apply in the present case. Again, the contribution of $\mathrm{H}+3$ jets at $\mathrm{NLO}$ grows rapidly for increasing $p_{T, \mathrm{H}}$ and becomes as large as $50 \%$ of the differential $\mathrm{H}+2$ jets cross section already around $160 \mathrm{GeV}$. This increases to a value of $70 \%$ if the $y$ - or $m_{j j}$-tagging strategy is used, stressing the effective LO nature of the $\mathrm{H}+2$ jets NLO calculation in this region. 

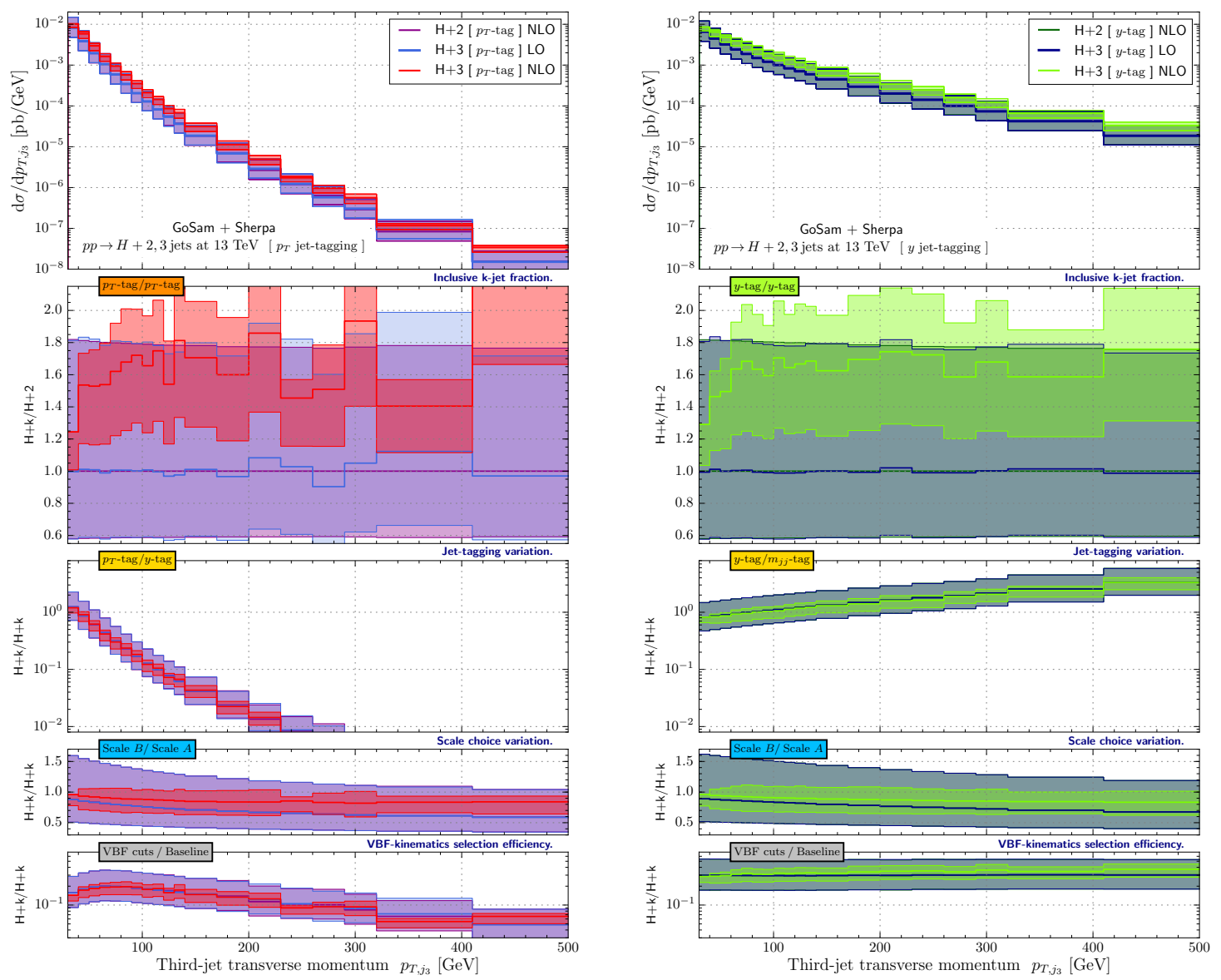

Figure 26. Impact of the $\mathrm{H}+3$ jets contribution described at NLO using scale choice B, cf. eq. (2.8b), on the transverse momentum spectrum of the hardest non-tagging jet, $p_{T, j_{3}}$, for both tag jet selections $p_{T}$ jet-tagging (left) and $y$ jet-tagging (right). LHC predictions at $13 \mathrm{TeV}$ centerof-mass energy are shown for several $\mathrm{H}+n$ jets processes imposing VBF kinematic requirements in addition to the baseline selection. Owing to the specific parameter settings, the distributions obtained for $\mathrm{H}+2$ jets at $\mathrm{NLO}$ and $\mathrm{H}+3$ jets at $\mathrm{LO}$ are identical except for small statistical fluctuations. Note that the figure layout is the same as in figure 21 whose caption provides more details.

For the inclusive tagging jets events, the transverse momentum spectra of the tagging jets and the leading non-tagging jet are shown in figures 25 and 26, respectively. Both of which are presented here as they are of great importance when discussing veto efficiencies on additional jet activity that occurs beyond the tagging jets. The new plots can be directly compared to figure 17. Apart from the general decrease in the cross section, the curves are qualitatively very similar, thus, the explanations given around figure 17 carry over to the current scenario that includes the VBF topological requirements. In particular, using the $p_{T}$-tag strategy, the turn-over in the $p_{T \text {,tag-jet }}$ distributions of the $\mathrm{H}+3$ jets calculations occurs, as before, at around twice the jet $p_{T}$ threshold. The $y$-tag scheme does not induce such a turn-over in the $p_{T}$ distribution. The corresponding differential three-jet fraction is 
hence found to rise only marginally from $60 \%$ at the jet threshold to $80 \%$ at $500 \mathrm{GeV}$. The entire $p_{T}$ range for $y$-tagged jets can therefore be expected to receive non-negligible higherorder corrections that appear beyond an NLO treatment for $\mathrm{H}+2$ jets. Comparing all three approaches, the $m_{j j}$-tag scheme is noticed to produce the hardest tagging-jet tails, followed by the $y$-tag scheme and lastly the $p_{T}$-tag one. By inspecting the plots at the bottom of figure 25, we find that the $y$-tagging approach also generates larger cut efficiencies of $\mathcal{O}(20 \%)$ that increase to $30 \%$ for higher jet multiplicity while they remain at around $15 \%$ in the $p_{T}$-tag case. The reason for this behavior lies in the event preselection of the $y$-tagging scheme, which favors final-state configurations of well separated jets in rapidity. This makes it easier to fulfill the VBF constraints, in particular the minimal constraint on $\Delta y_{j_{1}, j_{2}}$. In addition, this preselection also benefits from an increase of combinatorial options as soon as there are more than two jets in the final state. Turning to the $p_{T}$ distribution of the hardest of the untagged jets, we again immediately notice the clear difference in the steepness of the fall-off of the distributions predicted by the $p_{T}$-tagging scheme (figure 26 , left) and the $y$-tagging scheme (figure 26, right). As already discussed in section 3.5, $y$-tagging leaves us with a considerably harder spectrum, which even exceeds the, overall similar, $m_{j j}$-based prediction by a factor of two once $p_{T, j_{3}} \gtrsim 400 \mathrm{GeV}$. We hence note that the variable $p_{T, j_{3}}$ is one of the few quantities where the predictions of the two VBF-like tagging strategies exhibit larger deviations from each other. Moreover, in the $\mathrm{H}+2$ jets process, this variable is only described through the resolved real radiation contribution. As a result of this and the chosen parameter settings, the predictions from $\mathrm{H}+2$ jets at $\mathrm{NLO}$ and $\mathrm{H}+3$ jets at $\mathrm{LO}$ turn out to be identical (up to statistical fluctuations). Accordingly, the first ratio plots essentially display the differential $K$-factors signalling, as before at the baseline level, fairly large and uniform NLO corrections that vary between 1.2 and 2.0 in both tagging schemes. Although the $K$-factors are rather similar, the effect of the VBF cuts on the shape of the $p_{T, j_{3}}$ distribution is different. While the efficiency for $y$-tagging is almost constant, slightly increasing with larger transverse momenta, it drops significantly for $p_{T}$-tagging. The VBF selection therefore amplifies the apparent slope differences present at baseline level between the two schemes. Lastly, the choice regarding the scale's functional form does not introduce large effects in the jet $p_{T}$ spectra discussed here. Small distortions can be noticed but prove to be reduced for the NLO accurate $p_{T \text {,tag-jet }}$ and $p_{T, j_{3}}$ predictions.

The situation changes again for observables that are more directly affected by the VBF selection. In figure 27, we depict the inclusive rapidity distribution of the tagging jets, clearly showing the characteristic dip in the central region, which occurs as a consequence of satisfying the $\Delta y$ constraint between the two tagging jets. At the baseline level, the dip is absent, cf. figures 9 and 16 (to the right); the $\Delta y$ cut however forces the tag jets towards higher rapidities, causing the gap in the central region. This is demonstrated explicitly by the behavior of the differential cut efficiencies, shown in the ratio plots at the very bottom of figure 27. The precise shape of the gap strongly depends on the choice of the tagging scheme, but it hardly matters which of the scale definitions, A or B, has been employed: using $y$-tagging, the predictions are somewhat wider and more pronounced than using $p_{T^{-}}$ tagging, for which we find a slightly more effective central rapidity suppression. Again, $y$-tagging and $m_{j j}$-tagging give very similar results where for the latter scheme, the dip 

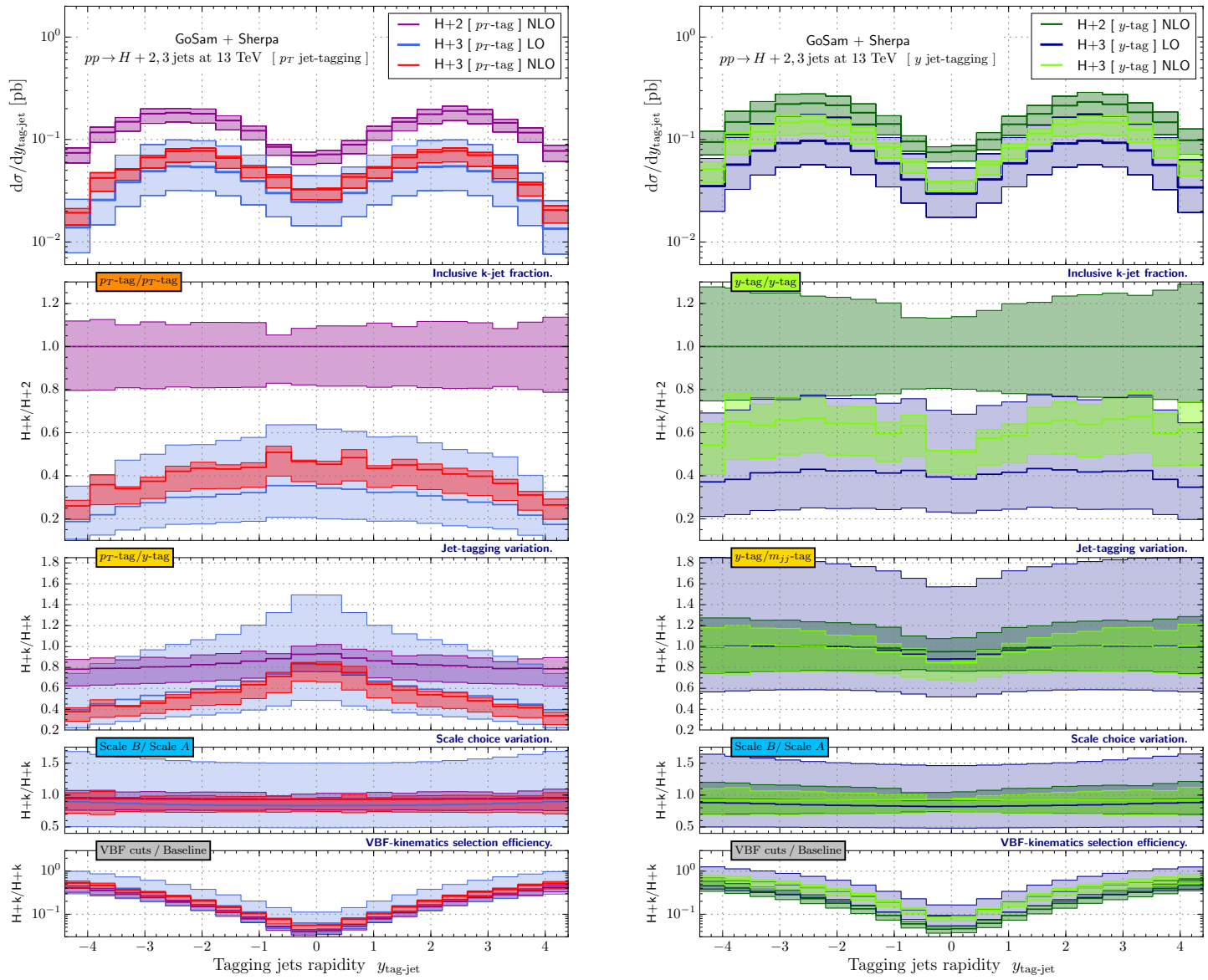

Figure 27. Impact of the $\mathrm{H}+3$ jets contribution described at NLO using scale choice B, cf. eq. (2.8b), on the inclusive tagging jets rapidity distribution, $y_{\text {tag-jet }}$, for both tag jet selections $p_{T}$ jet-tagging (left) and $y$ jet-tagging (right). LHC predictions at $13 \mathrm{TeV}$ center-of-mass energy are shown for several $\mathrm{H}+n$ jets processes imposing VBF kinematic requirements in addition to the baseline selection. Note that the figure layout is the same as in figure 21 whose caption provides more details.

turns out to be slightly more populated. Apart from the general fact that $R_{3 / 2}^{\left(p_{T}\right)} \lesssim R_{3 / 2}^{(y)}$, a clear difference between the two schemes becomes apparent by comparing the behavior in the central and peak regions of the three-jet fraction plots. Using $p_{T}$-tagging, central rapidities are found to be most susceptible to three-jet corrections exhibiting values of $R_{3 / 2}^{\left(p_{T}\right)}$ that get as large as $40 \%$. On the contrary, the same region is affected least by the $y$-tagging description of $\mathrm{H}+3$ jets. The largest effects ranging up to $R_{3 / 2}^{(y)} \sim 0.6$ emerge in the peak regions of the $\mathrm{H}+2$ jets NLO prediction, as a direct consequence of strictly tagging the most forward and backward jets.

As exemplified by figure 28, a rapidity gap does not emerge neither in the rapidity distribution of any non-tagging jet nor in the rapidity distribution of the Higgs boson (which we do not show here). This happens for two reasons: first, the VBF selection only 

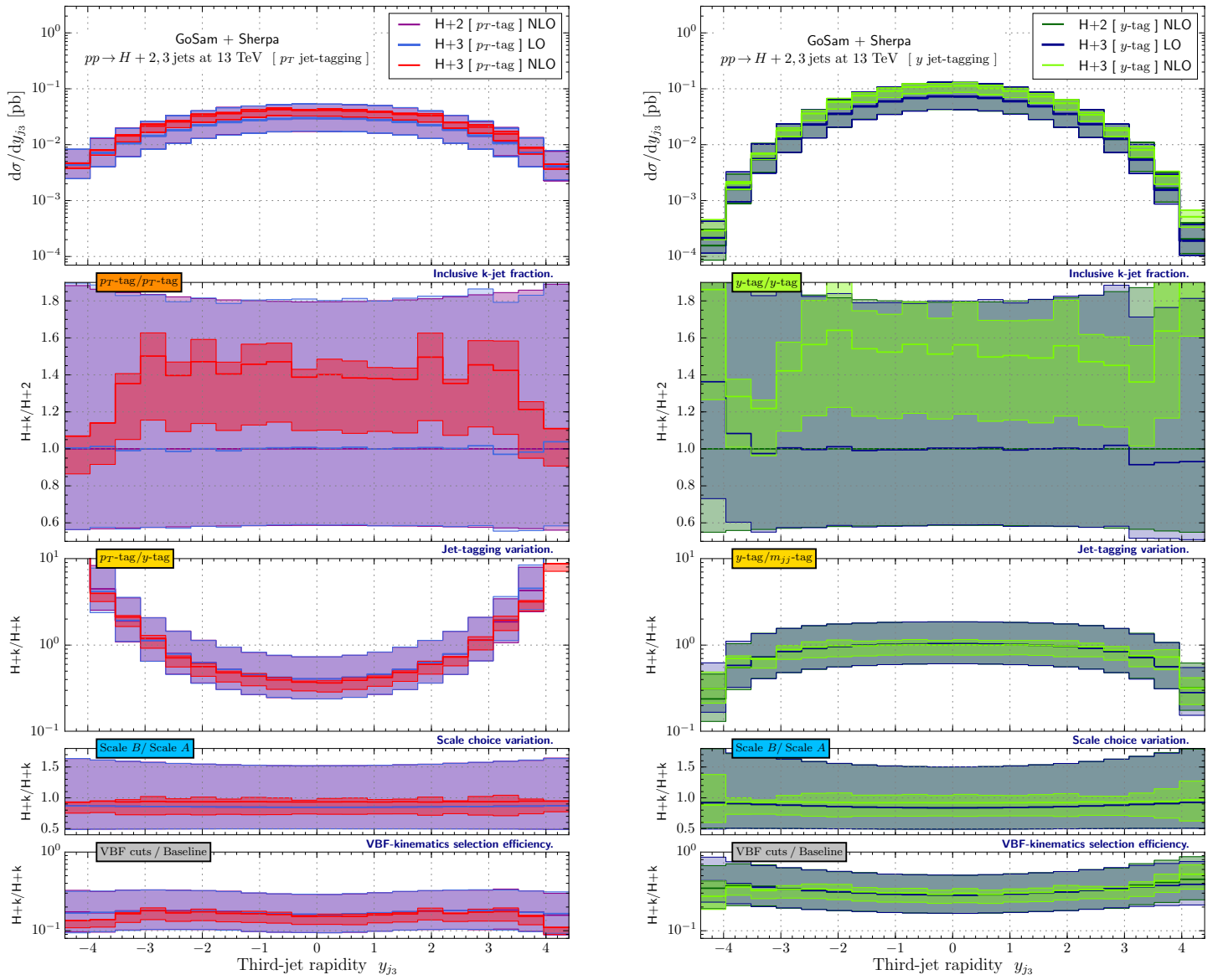

Figure 28. Impact of the $\mathrm{H}+3$ jets contribution described at NLO using scale choice B, cf. eq. (2.8b), on the rapidity spectrum of the hardest non-tagging jet, $y_{j_{3}}$, for both tag jet selections $p_{T}$ jet-tagging (left) and $y$ jet-tagging (right). LHC predictions at $13 \mathrm{TeV}$ center-of-mass energy are shown for several $\mathrm{H}+n$ jets processes imposing VBF kinematic requirements in addition to the baseline selection. Owing to the specific parameter settings, the distributions generated by the $\mathrm{H}+$ 2 jets NLO calculation and the $\mathrm{H}+3$ jets $\mathrm{LO}$ calculation are identical except for small statistical fluctuations. Note that the figure layout is the same as in figure 21 whose caption provides more details.

acts on the two tagging jets and, secondly, unlike Higgs boson production via weak boson fusion, the production via gluon fusion comprises topologies with color connections between all colored partons, including those where the color exchange occurs between incoming partons. Still one encounters significant shape changes in the predictions based on the different tagging strategies. Using $p_{T}$-tagging, we obtain fairly wide rapidity distributions. They steepen once we switch to the $m_{j j}$ scheme, and turn out to be steepest for $y$-tagging. In other words, in the $y$-tagging scheme, the third jet appears much more central than for $p_{T}$-tagging and only somewhat more central compared to $m_{j j}$-tagging. The reason for this pattern is that the VBF-like tagging schemes support configurations where the hardest jet does not matter in terms of $y$ ordering. Of these configurations there are many showing the 

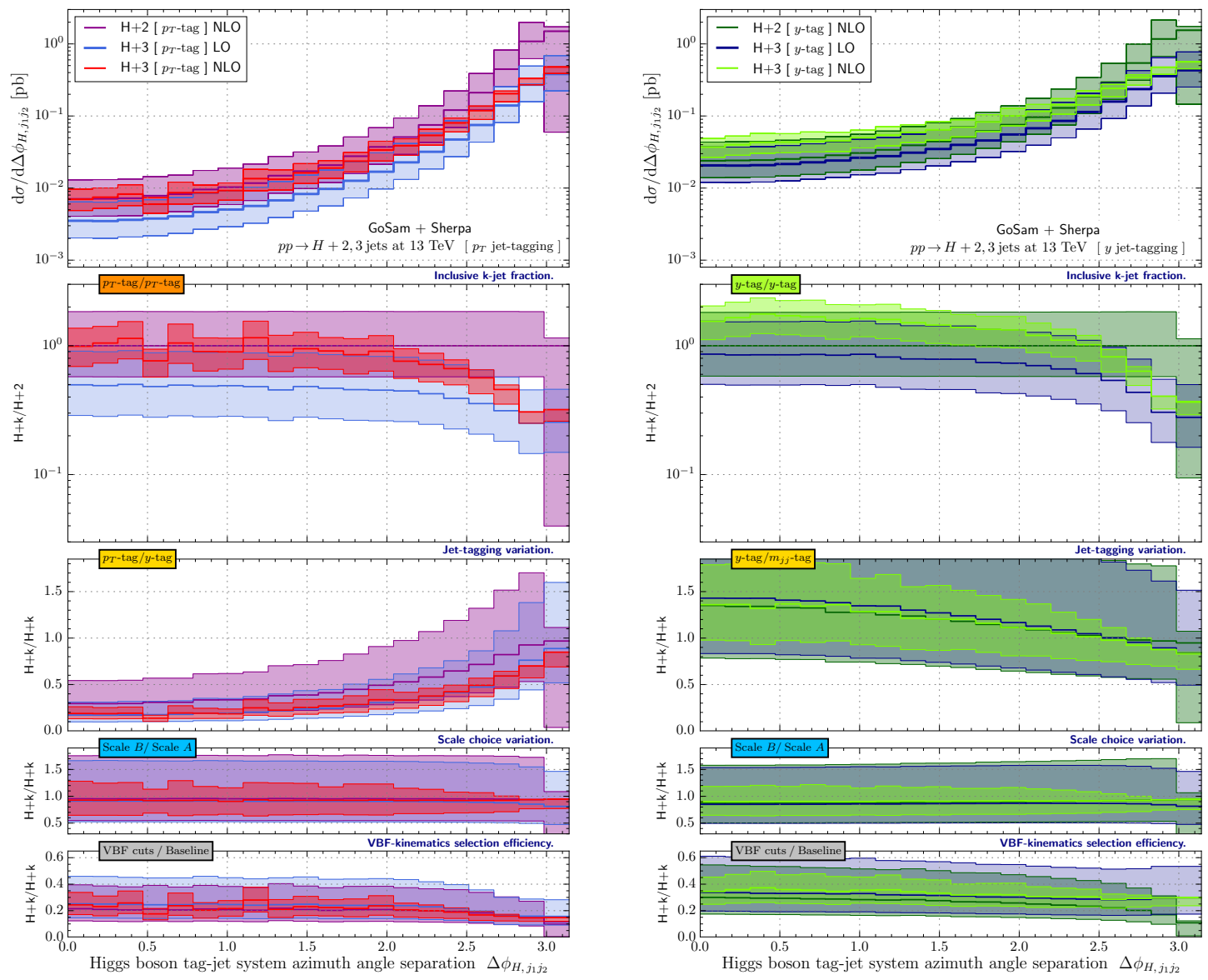

Figure 29. Impact of the $\mathrm{H}+3$ jets contribution described at NLO using scale choice B, cf. eq. (2.8b), on the azimuthal separation of the Higgs boson and the tagging jet system, $\Delta \phi_{\mathrm{H}, j_{1} j_{2}}$, for both tag jet selections $p_{T}$ jet-tagging (left) and $y$ jet-tagging (right). LHC predictions at $13 \mathrm{TeV}$ center-of-mass energy are shown for several $\mathrm{H}+n$ jets processes imposing VBF kinematic requirements in addition to the baseline selection. Note that the figure layout is the same as in figure 21 whose caption provides more details.

hardest jet to be produced centrally, sandwiched by the others, and it is these configurations that substantially shape the $y$ distribution of the leading untagged jet. Similar to the case of the $p_{T, j_{3}}$ variable, the first ratio plot in figure 28 again turns into an illustration of the differential $K$-factor for $\mathrm{H}+3$ jets production, this time as a function of $y_{j_{3}}$. In both schemes, the $K$-factors are almost constant over a broad range of rapidities (from -3.0 to 3.0), while they differ regarding the size of the correction, amounting to $\sim 1.4$ for $p_{T}$-tagging and $\lesssim 1.6$ for $y$-tagging. As before, choosing between scale A or B is almost inconsequential.

We finish by discussing two observables, which relate the Higgs boson to the tagging jets. In figure 29, we have plotted the azimuthal separation between the Higgs boson and the dijet system defined by the two tagging jets. The $p_{T}$-tag based predictions can be compared with the ones in figure 5 (to the right), where the same observable is shown applying the baseline cuts only. Again, many of the details given there carry over to this 

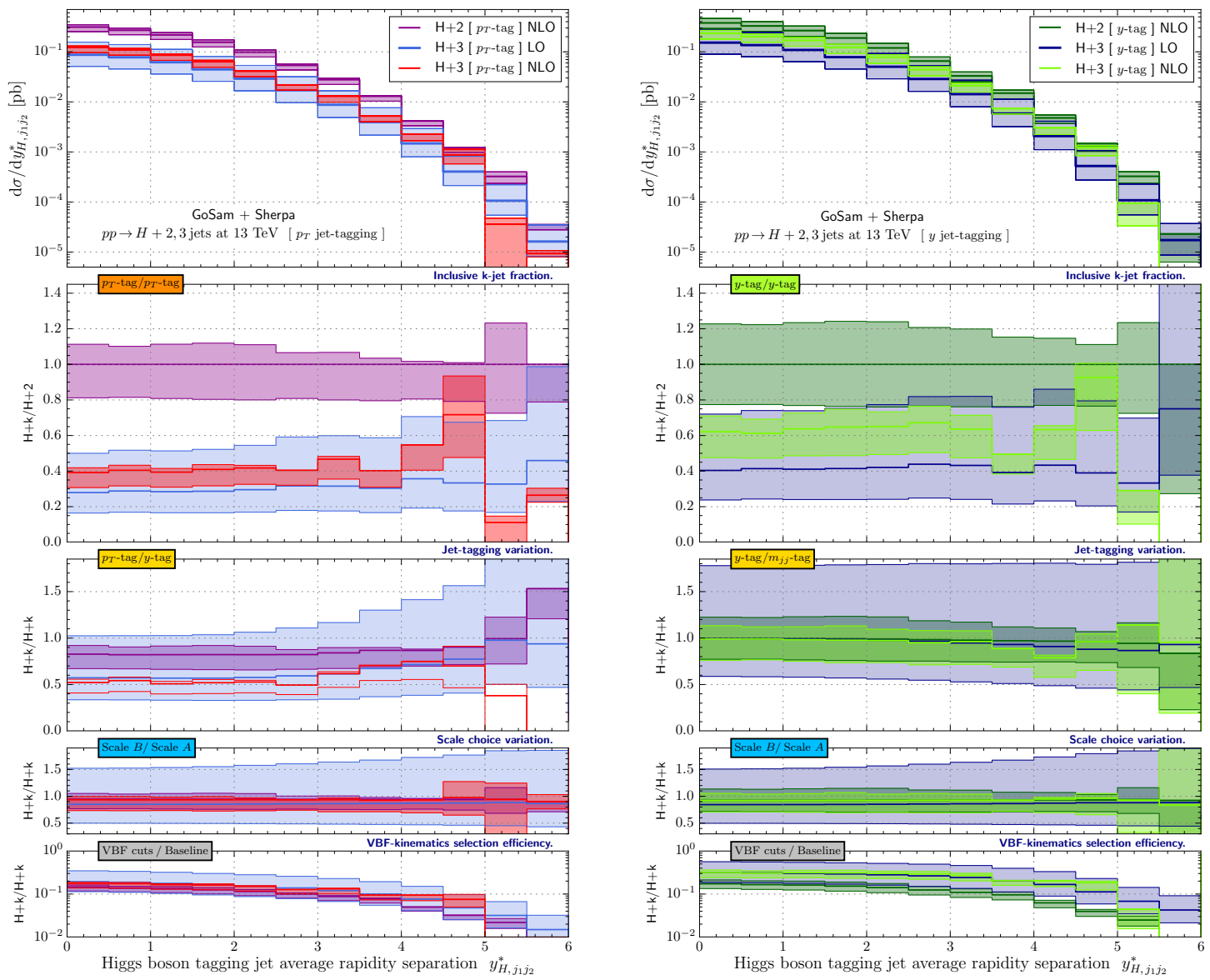

Figure 30. Impact of the $\mathrm{H}+3$ jets contribution described at NLO using scale choice B, cf. eq. (2.8b), on the rapidity separation of the Higgs boson and the average of the tagging jets (denoted as $y_{\mathrm{H}, j_{1} j_{2}}^{*}$ ) for both tag jet selections $p_{T}$ jet-tagging (left) and $y$ jet-tagging (right). LHC predictions at $13 \mathrm{TeV}$ center-of-mass energy are shown for several $\mathrm{H}+n$ jets processes imposing VBF kinematic requirements in addition to the baseline selection. Note that the figure layout is the same as in figure 21 whose caption provides more details.

case. As illustrated by the bottom ratios, it is clear that the shape of the predictions is very similar apart from a slightly milder increase of the curves towards the back-to-back configuration. The large scale uncertainty in the $\mathrm{H}+2$ jets curve is a reminder of the fact that (by construction of the observable) this prediction is no better than a LO description can provide. Therefore, the contributions coming from $\mathrm{H}+3$ jets NLO corrections are particularly large and need to be taken into account for a precise theoretical prediction. It however is irrelevant whether we obtain these predictions from using scale choice A or scale choice B. We also note that all considerations above hold for both $p_{T}$-tagging as well as $y$ tagging. However, by directly comparing the outcomes of the tagging schemes, we observe differences in the occurrence of small-angle configurations. The predictions from $y$-tagging decline more slowly towards small angles than those given by the other scenarios. While all 
tagging schemes favor, to similar extent, the configurations where the Higgs boson recoils against both tagging jets, the way their $\Delta \phi_{\mathrm{H}, j_{1} j_{2}}$ predictions level off at small angles is very different, suppressed with respect to the $y$-tag scheme by factors of $\sim 5.0$ and $\sim 1.35$ for the $p_{T}$-tag and $m_{j j}$-tag scenario, respectively. Thus, the $y$-tagging generates a noticeably larger number of events where the Higgs boson and the tagging jets recoil against the rest of the event. Again, this is more easily understood by inspecting the tagging procedure in terms of the third-jet selection. It is clear that the procedures will leave us with more or less energetic jets to recoil against and, thus, more or less opportunities for the Higgs boson plus tagging jet system to be boosted into the same direction.

Finally, figure 30 shows again $y_{\mathrm{H}, j_{1} j_{2}}^{*}$, defined in eq. (3.6). Compared to the results obtained at baseline level, see figure 18 (to the right), the distributions fall off a bit faster for very large rapidity separations, independently of the tagging method, and, as before, the choice of using scale A or B in our calculations. Another difference that is worthwhile to be pointed out lies in the approximate uniformity of the differential three-jet fractions, achieved as a result of requiring VBF-like final-state topologies. Before any of the VBF cuts has come in effect, the $R_{3 / 2}$ ratios were falling towards larger $y_{\mathrm{H}, j_{1} j_{2}}^{*}$. Taking the VBF cuts into account, we find the values for $R_{3 / 2}$ to be increased amounting respectively to $\mathcal{O}(40 \%)$ and $\mathcal{O}(60 \%)$ for the $p_{T}$-tagging and $y$-tagging scheme. In addition, one may find it appealing to notice a tendency for further $R_{3 / 2}$ increase in both schemes (to $\mathcal{O}(70 \%)$ and $\mathcal{O}(90 \%)$, respectively) once $y_{\mathrm{H}, j_{1} j_{2}}^{*} \gtrsim 4$. Nonetheless, the results of figure 30 make it clear that the contribution arising from the NLO corrections regarding $\mathrm{H}+3$ jets becomes even more important after the application of VBF cuts. Again, the larger three-jet fractions appear in the VBF-like jet-tagging schemes. Qualitatively, we however see that all tagging schemes predict the same shape for $y_{\mathrm{H}, j_{1} j_{2}}^{*} \lesssim 4$ where both $y$-tagging as well as $m_{j j}$-tagging allow for a few more events at even larger $y_{\mathrm{H}, j_{1} j_{2}}^{*}$. We also notice that the ratio between $\mathrm{VBF}$ and baseline cuts stabilizes in a $\mathrm{H}+3$ jets $\mathrm{NLO}$ calculation as compared to the respective $\mathrm{LO}$ one and the $\mathrm{NLO}$ calculation for $\mathrm{H}+2$ jets.

\section{Conclusions}

Gluon fusion is the dominant production mechanism for a Standard Model Higgs boson at the LHC. The production of a Higgs boson in gluon fusion in association with jets also constitutes an irreducible background to the vector boson fusion mechanism. Reliable predictions for the Higgs boson plus jets processes are therefore indispensable for a precise determination of the Higgs boson couplings and its properties in the VBF signal.

In this paper we have presented a detailed phenomenological analysis of the gluon fusion contribution to Higgs boson plus jets were we focused on two and three additional jets in the final state. The calculations have been performed in the limit of an infinitely heavy top quark, at next-to-leading order in QCD. Results for LHC collision energies of $8 \mathrm{TeV}$ and $13 \mathrm{TeV}$ have been obtained by the combination of the fully automated tools GoSam and Sherpa. The numerical results have been generated in two steps. First we have produced sets of Ntuples for the two energies and the three different jet multiplicities with a minimal set of kinematic requirements, which in a second step, have been analyzed for the particular scenarios. The entire set of Ntuples will be made publicly available. 
We have investigated two major scenarios, one defined by applying only basic selection cuts, and the second by applying the considerably more constraining VBF cuts where we also investigated alternative tagging jet selections. We found that independent of the final state jet multiplicity the NLO QCD corrections remain sizeable and are therefore an important prerequisite for a reliable prediction. In particular in the VBF scenario, for both the two jet as well as the three jet bin, the additional jet production accounts for a considerable fraction of the total cross section which means that the results, to a large extent, are only given with leading order accuracy. However, if one considers a veto on the third jet in a two jet calculation, this again would introduce large theoretical uncertainties. Therefore the calculation of the three jet process with NLO accuracy provides important information also for the exclusive two jet result.

For inclusive observables, i.e. observables that are not a priori dependent on a specific number of jets, such as the transverse momentum of the Higgs boson, we find that the higher jet multiplicities are important for the correct description of the shape of the observables. In particular in the tails of the distributions, which might be sensitive to new physics, they can make up the dominant contribution. Also here, the inclusion of the NLO corrections of $\mathrm{H}+3$ jets leads to an improvement of the theoretical prediction.

We discussed a large variety of differential distributions which are suitable to distinguish the gluon fusion process from that of the vector boson fusion. Some of these observables are also used as input variables for the boosted decision trees in the experiment. We particularly described the effects of a third jet as well as the impact of the NLO corrections.

Further improvements could certainly be achieved by providing a merged NLO result of the different jet multiplicities, but also through the inclusion of top-quark mass effects as well as the matching of the $\mathrm{H}+3$ jets NLO result with a parton shower. Due to the complexity of these improvements they are however beyond the scope of this paper and we leave them for future work.

\section{Acknowledgments}

We thank Joey Huston for several fruitful discussions and Pierpaolo Mastrolia for his encouragement during several stages of this project. For this research project, we made extensive use of the computation resources of the RechenZentrum Garching (RZG). We are thankful to the RZG for providing us with these resources. This work was supported by the US Department of Energy under contract DE-AC02-76SF00515 and the Swiss National Foundation (SNF) under contract PP00P2-128552. M.S. would like to thank the University Duisburg-Essen for the kind hospitality during the final stages of the project. The work of G.L. and V.Y. was supported by the Alexander von Humboldt Foundation, in the framework of the Sofja Kovaleskaja Award 2010, endowed by the German Federal Ministry of Education and Research.

Open Access. This article is distributed under the terms of the Creative Commons Attribution License (CC-BY 4.0), which permits any use, distribution and reproduction in any medium, provided the original author(s) and source are credited. 


\section{References}

[1] ATLAS collaboration, Observation of a new particle in the search for the Standard Model Higgs boson with the ATLAS detector at the LHC, Phys. Lett. B 716 (2012) 1 [arXiv: 1207.7214] [INSPIRE].

[2] CMS collaboration, Observation of a new boson at a mass of $125 \mathrm{GeV}$ with the CMS experiment at the LHC, Phys. Lett. B 716 (2012) 30 [arXiv:1207.7235] [INSPIRE].

[3] ATLAS collaboration, Evidence for the spin-0 nature of the Higgs boson using ATLAS data, Phys. Lett. B 726 (2013) 120 [arXiv:1307.1432] [INSPIRE].

[4] CMS collaboration, Measurement of Higgs boson production and properties in the $W W$ decay channel with leptonic final states, JHEP 01 (2014) 096 [arXiv:1312.1129] [INSPIRE].

[5] CMS collaboration, Measurement of the properties of a Higgs boson in the four-lepton final state, Phys. Rev. D 89 (2014) 092007 [arXiv: 1312.5353] [INSPIRE].

[6] ATLAS collaboration, Measurements of Higgs boson production and couplings in diboson final states with the ATLAS detector at the LHC, Phys. Lett. B 726 (2013) 88 [Erratum ibid. B 734 (2014) 406] [arXiv:1307.1427] [INSPIRE].

[7] ATLAS collaboration, Measurement of Higgs boson production in the diphoton decay channel in pp collisions at center-of-mass energies of 7 and 8 TeV with the ATLAS detector, Phys. Rev. D 90 (2014) 112015 [arXiv:1408.7084] [INSPIRE].

[8] ATLAS collaboration, Measurements of fiducial and differential cross sections for Higgs boson production in the diphoton decay channel at $\sqrt{s}=8$ TeV with ATLAS, JHEP 09 (2014) 112 [arXiv:1407.4222] [INSPIRE].

[9] ATLAS collaboration, Measurement of the Higgs boson mass from the $H \rightarrow \gamma \gamma$ and $H \rightarrow Z Z^{*} \rightarrow 4 \ell$ channels with the ATLAS detector using $25 \mathrm{fb}^{-1}$ of pp collision data, Phys. Rev. D 90 (2014) 052004 [arXiv: 1406.3827] [INSPIRE].

[10] ATLAS collaboration, Search for Higgs boson decays to a photon and a $Z$ boson in $p p$ collisions at $\sqrt{s}=7$ and 8 TeV with the ATLAS detector, Phys. Lett. B 732 (2014) 8 [arXiv: 1402.3051] [INSPIRE].

[11] ATLAS collaboration, Evidence for the Higgs-boson Yukawa coupling to $\tau$ leptons with the ATLAS detector, JHEP 04 (2015) 117 [arXiv: 1501.04943] [INSPIRE].

[12] ATLAS collaboration, Observation and measurement of Higgs boson decays to $W W^{*}$ with the ATLAS detector, Phys. Rev. D 92 (2015) 012006 [arXiv:1412.2641] [INSPIRE].

[13] CMS collaboration, Evidence for the $125 \mathrm{GeV}$ Higgs boson decaying to a pair of $\tau$ leptons, JHEP 05 (2014) 104 [arXiv: 1401.5041] [INSPIRE].

[14] CMS collaboration, Evidence for the direct decay of the $125 \mathrm{GeV}$ Higgs boson to fermions, Nature Phys. 10 (2014) 557 [arXiv:1401.6527] [INSPIRE].

[15] CMS collaboration, Observation of the diphoton decay of the Higgs boson and measurement of its properties, Eur. Phys. J. C 74 (2014) 3076 [arXiv:1407.0558] [INSPIRE].

[16] CMS collaboration, Precise determination of the mass of the Higgs boson and tests of compatibility of its couplings with the Standard Model predictions using proton collisions at 7 and 8 TeV, Eur. Phys. J. C 75 (2015) 212 [arXiv:1412.8662] [InSPIRE].

[17] F. Englert and R. Brout, Broken symmetry and the mass of gauge vector mesons, Phys. Rev. Lett. 13 (1964) 321 [INSPIRE]. 
[18] P.W. Higgs, Broken symmetries and the masses of gauge bosons, Phys. Rev. Lett. 13 (1964) 508 [INSPIRE].

[19] G.S. Guralnik, C.R. Hagen and T.W.B. Kibble, Global conservation laws and massless particles, Phys. Rev. Lett. 13 (1964) 585 [InSPIRE].

[20] T.W.B. Kibble, Symmetry breaking in non-Abelian gauge theories, Phys. Rev. 155 (1967) 1554 [INSPIRE].

[21] R.N. Cahn and S. Dawson, Production of very massive Higgs bosons, Phys. Lett. B 136 (1984) 196 [Erratum ibid. B 138 (1984) 464] [InSPIRE].

[22] G.L. Kane, W.W. Repko and W.B. Rolnick, The effective $W^{ \pm}, Z^{0}$ approximation for high-energy collisions, Phys. Lett. B 148 (1984) 367 [INSPIRE].

[23] D.L. Rainwater and D. Zeppenfeld, Searching for $H \rightarrow \gamma \gamma$ in weak boson fusion at the LHC, JHEP 12 (1997) 005 [hep-ph/9712271] [INSPIRE].

[24] D.L. Rainwater, D. Zeppenfeld and K. Hagiwara, Searching for $H \rightarrow \tau^{+} \tau^{-}$in weak boson fusion at the CERN LHC, Phys. Rev. D 59 (1998) 014037 [hep-ph/9808468] [INSPIRE].

[25] D.L. Rainwater and D. Zeppenfeld, Observing $H \rightarrow W^{*} W^{*} \rightarrow e^{ \pm} \mu \mp \not p_{T}$ in weak boson fusion with dual forward jet tagging at the CERN LHC, Phys. Rev. D 60 (1999) 113004 [Erratum ibid. D 61 (2000) 099901] [hep-ph/9906218] [INSPIRE].

[26] T. Plehn, D.L. Rainwater and D. Zeppenfeld, Probing the MSSM Higgs sector via weak boson fusion at the LHC, Phys. Lett. B 454 (1999) 297 [hep-ph/9902434] [InSPIRE].

[27] O.J.P. Eboli and D. Zeppenfeld, Observing an invisible Higgs boson, Phys. Lett. B 495 (2000) 147 [hep-ph/0009158] [INSPIRE].

[28] T. Han, G. Valencia and S. Willenbrock, Structure function approach to vector boson scattering in pp collisions, Phys. Rev. Lett. 69 (1992) 3274 [hep-ph/9206246] [INSPIRE].

[29] T. Figy, C. Oleari and D. Zeppenfeld, Next-to-leading order jet distributions for Higgs boson production via weak boson fusion, Phys. Rev. D 68 (2003) 073005 [hep-ph/0306109] [INSPIRE].

[30] P. Bolzoni, F. Maltoni, S.-O. Moch and M. Zaro, Higgs production via vector-boson fusion at NNLO in QCD, Phys. Rev. Lett. 105 (2010) 011801 [arXiv:1003.4451] [InSPIRE].

[31] M. Ciccolini, A. Denner and S. Dittmaier, Strong and electroweak corrections to the production of Higgs +2 jets via weak interactions at the LHC, Phys. Rev. Lett. 99 (2007) 161803 [arXiv: 0707.0381] [INSPIRE].

[32] M. Ciccolini, A. Denner and S. Dittmaier, Electroweak and QCD corrections to Higgs production via vector-boson fusion at the LHC, Phys. Rev. D 77 (2008) 013002 [arXiv:0710.4749] [INSPIRE].

[33] S. Dawson, Radiative corrections to Higgs boson production, Nucl. Phys. B 359 (1991) 283 [INSPIRE].

[34] A. Djouadi, M. Spira and P.M. Zerwas, Production of Higgs bosons in proton colliders: QCD corrections, Phys. Lett. B 264 (1991) 440 [INSPIRE].

[35] D. de Florian, M. Grazzini and Z. Kunszt, Higgs production with large transverse momentum in hadronic collisions at next-to-leading order, Phys. Rev. Lett. 82 (1999) 5209 [hep-ph/9902483] [INSPIRE]. 
[36] J.M. Campbell, R.K. Ellis and C. Williams, Hadronic production of a Higgs boson and two jets at next-to-leading order, Phys. Rev. D 81 (2010) 074023 [arXiv:1001.4495] [INSPIRE].

[37] J.M. Campbell, R.K. Ellis and G. Zanderighi, Next-to-leading order Higgs +2 jet production via gluon fusion, JHEP 10 (2006) 028 [hep-ph/0608194] [INSPIRE].

[38] V. Ravindran, J. Smith and W.L. Van Neerven, Next-to-leading order QCD corrections to differential distributions of Higgs boson production in hadron hadron collisions, Nucl. Phys. B 634 (2002) 247 [hep-ph/0201114] [INSPIRE].

[39] L.J. Dixon and Y. Sofianatos, Analytic one-loop amplitudes for a Higgs boson plus four partons, JHEP 08 (2009) 058 [arXiv:0906.0008] [INSPIRE].

[40] V. Del Duca, A. Frizzo and F. Maltoni, Higgs boson production in association with three jets, JHEP 05 (2004) 064 [hep-ph/0404013] [INSPIRE].

[41] L.J. Dixon, E.W.N. Glover and V.V. Khoze, MHV rules for Higgs plus multi-gluon amplitudes, JHEP 12 (2004) 015 [hep-th/0411092] [INSPIRE].

[42] S.D. Badger, E.W.N. Glover and V.V. Khoze, MHV rules for Higgs plus multi-parton amplitudes, JHEP 03 (2005) 023 [hep-th/0412275] [INSPIRE].

[43] R.K. Ellis, W.T. Giele and G. Zanderighi, Virtual QCD corrections to Higgs boson plus four parton processes, Phys. Rev. D 72 (2005) 054018 [Erratum ibid. D 74 (2006) 079902] [hep-ph/0506196] [INSPIRE].

[44] R.K. Ellis, W.T. Giele and G. Zanderighi, Semi-numerical evaluation of one-loop corrections, Phys. Rev. D 73 (2006) 014027 [hep-ph/0508308] [INSPIRE].

[45] C.F. Berger, V. Del Duca and L.J. Dixon, Recursive construction of Higgs-plus-multiparton loop amplitudes: the last of the $\phi$-nite loop amplitudes, Phys. Rev. D 74 (2006) 094021 [Erratum ibid. D 76 (2007) 099901] [hep-ph/0608180] [INSPIRE].

[46] S.D. Badger and E.W.N. Glover, One-loop helicity amplitudes for $H \rightarrow$ gluons: the all-minus configuration, Nucl. Phys. Proc. Suppl. 160 (2006) 71 [hep-ph/0607139] [INSPIRE].

[47] S.D. Badger, E.W.N. Glover and K. Risager, One-loop $\phi$-MHV amplitudes using the unitarity bootstrap, JHEP 07 (2007) 066 [arXiv:0704.3914] [INSPIRE].

[48] E.W.N. Glover, P. Mastrolia and C. Williams, One-loop $\phi$-MHV amplitudes using the unitarity bootstrap: the general helicity case, JHEP 08 (2008) 017 [arXiv:0804.4149] [INSPIRE].

[49] S. Badger, E.W. Nigel Glover, P. Mastrolia and C. Williams, One-loop Higgs plus four gluon amplitudes: full analytic results, JHEP 01 (2010) 036 [arXiv:0909.4475] [INSPIRE].

[50] S. Badger, J.M. Campbell, R.K. Ellis and C. Williams, Analytic results for the one-loop NMHV Hqqgg amplitude, JHEP 12 (2009) 035 [arXiv:0910.4481] [INSPIRE].

[51] H. van Deurzen et al., $N L O Q C D$ corrections to the production of Higgs plus two jets at the LHC, Phys. Lett. B 721 (2013) 74 [arXiv:1301.0493] [INSPIRE].

[52] J.M. Campbell, R.K. Ellis, R. Frederix, P. Nason, C. Oleari and C. Williams, NLO Higgs boson production plus one and two jets using the POWHEG BOX, MadGraph4 and MCFM, JHEP 07 (2012) 092 [arXiv: 1202.5475] [INSPIRE].

[53] S. Höche, F. Krauss and M. Schönherr, Uncertainties in MEPS@NLO calculations of $h+$ jets, Phys. Rev. D 90 (2014) 014012 [arXiv:1401.7971] [INSPIRE]. 
[54] G. Cullen et al., Next-to-leading-order QCD corrections to Higgs boson production plus three jets in gluon fusion, Phys. Rev. Lett. 111 (2013) 131801 [arXiv:1307.4737] [INSPIRE].

[55] P. Mastrolia, E. Mirabella and T. Peraro, Integrand reduction of one-loop scattering amplitudes through Laurent series expansion, JHEP 06 (2012) 095 [Erratum ibid. 11 (2012) 128] [arXiv: 1203.0291] [INSPIRE].

[56] H. van Deurzen, G. Luisoni, P. Mastrolia, E. Mirabella, G. Ossola and T. Peraro, Multi-leg one-loop massive amplitudes from integrand reduction via Laurent expansion, JHEP 03 (2014) 115 [arXiv: 1312.6678] [inSPIRE].

[57] T. Peraro, Ninja: automated integrand reduction via Laurent expansion for one-loop amplitudes, Comput. Phys. Commun. 185 (2014) 2771 [arXiv:1403.1229] [INSPIRE].

[58] J.R. Andersen et al., Les Houches 2013 - physics at TeV colliders: Standard Model working group report, arXiv:1405.1067 [INSPIRE].

[59] R. Boughezal, F. Caola, K. Melnikov, F. Petriello and M. Schulze, Higgs boson production in association with a jet at next-to-next-to-leading order in perturbative QCD, JHEP 06 (2013) 072 [arXiv: 1302.6216] [inSPIRE].

[60] X. Chen, T. Gehrmann, E.W.N. Glover and M. Jaquier, Precise QCD predictions for the production of Higgs + jet final states, Phys. Lett. B 740 (2015) 147 [arXiv:1408.5325] [INSPIRE].

[61] R. Boughezal, F. Caola, K. Melnikov, F. Petriello and M. Schulze, Higgs boson production in association with a jet at next-to-next-to-leading order, Phys. Rev. Lett. 115 (2015) 082003 [arXiv: 1504.07922] [INSPIRE].

[62] R. Boughezal, C. Focke, W. Giele, X. Liu and F. Petriello, Higgs boson production in association with a jet at NNLO using jettiness subtraction, Phys. Lett. B 748 (2015) 5 [arXiv: 1505. 03893] [INSPIRE].

[63] R.V. Harlander and W.B. Kilgore, Next-to-next-to-leading order Higgs production at hadron colliders, Phys. Rev. Lett. 88 (2002) 201801 [hep-ph/0201206] [INSPIRE].

[64] C. Anastasiou, K. Melnikov and F. Petriello, Fully differential Higgs boson production and the di-photon signal through next-to-next-to-leading order, Nucl. Phys. B 724 (2005) 197 [hep-ph/0501130] [INSPIRE].

[65] M. Grazzini, NNLO predictions for the Higgs boson signal in the $H \rightarrow W W \rightarrow \ell \nu \ell \nu$ and $H \rightarrow Z Z \rightarrow 4 \ell$ decay channels, JHEP 02 (2008) 043 [arXiv:0801.3232] [INSPIRE].

[66] K. Hamilton, P. Nason, E. Re and G. Zanderighi, NNLOPS simulation of Higgs boson production, JHEP 10 (2013) 222 [arXiv:1309.0017] [INSPIRE].

[67] S. Höche, Y. Li and S. Prestel, Higgs-boson production through gluon fusion at NNLO QCD with parton showers, Phys. Rev. D 90 (2014) 054011 [arXiv: 1407.3773] [INSPIRE].

[68] C. Anastasiou, C. Duhr, F. Dulat, F. Herzog and B. Mistlberger, Real-virtual contributions to the inclusive Higgs cross-section at $N^{3}$ LO, JHEP 12 (2013) 088 [arXiv:1311.1425] [INSPIRE].

[69] W.B. Kilgore, One-loop single-real-emission contributions to $p p \rightarrow H+X$ at next-to-next-to-next-to-leading order, Phys. Rev. D 89 (2014) 073008 [arXiv:1312.1296] [INSPIRE]. 
[70] R.D. Ball, M. Bonvini, S. Forte, S. Marzani and G. Ridolfi, Higgs production in gluon fusion beyond NNLO, Nucl. Phys. B 874 (2013) 746 [arXiv:1303.3590] [InSPIRE].

[71] Y. Li and H.X. Zhu, Single soft gluon emission at two loops, JHEP 11 (2013) 080 [arXiv: 1309.4391] [INSPIRE].

[72] C. Duhr and T. Gehrmann, The two-loop soft current in dimensional regularization, Phys. Lett. B 727 (2013) 452 [arXiv: 1309.4393] [INSPIRE].

[73] C. Anastasiou et al., Higgs boson gluon-fusion production at threshold in $N^{3} L O Q C D$, Phys. Lett. B 737 (2014) 325 [arXiv: 1403.4616] [INSPIRE].

[74] C. Anastasiou et al., Higgs boson gluon-fusion production beyond threshold in $N^{3} L O Q C D$, JHEP 03 (2015) 091 [arXiv:1411.3584] [INSPIRE].

[75] Y. Li, A. von Manteuffel, R.M. Schabinger and H.X. Zhu, $N^{3} L O$ Higgs boson and Drell-Yan production at threshold: the one-loop two-emission contribution, Phys. Rev. D 90 (2014) 053006 [arXiv: 1404.5839] [inSPIRE].

[76] T. Ahmed, M. Mandal, N. Rana and V. Ravindran, Rapidity distributions in Drell-Yan and Higgs productions at threshold to third order in QCD, Phys. Rev. Lett. 113 (2014) 212003 [arXiv: 1404.6504] [INSPIRE].

[77] C. Anastasiou, C. Duhr, F. Dulat, F. Herzog and B. Mistlberger, Higgs boson gluon-fusion production in QCD at three loops, Phys. Rev. Lett. 114 (2015) 212001 [arXiv:1503.06056] [INSPIRE].

[78] J.R. Andersen and J.M. Smillie, The factorisation of the t-channel pole in quark-gluon scattering, Phys. Rev. D 81 (2010) 114021 [arXiv:0910.5113] [INSPIRE].

[79] J.R. Andersen and J.M. Smillie, Multiple jets at the LHC with high energy jets, JHEP 06 (2011) 010 [arXiv: 1101.5394] [INSPIRE].

[80] Z. Bern et al., Next-to-leading order W+5-jet production at the LHC, Phys. Rev. D 88 (2013) 014025 [arXiv: 1304.1253] [INSPIRE].

[81] Z. Bern et al., Extrapolating $W$-associated jet-production ratios at the LHC, Phys. Rev. D 92 (2015) 014008 [arXiv:1412.4775] [INSPIRE].

[82] E. Gerwick, T. Plehn, S. Schumann and P. Schichtel, Scaling patterns for QCD jets, JHEP 10 (2012) 162 [arXiv:1208.3676] [INSPIRE].

[83] E. Gerwick, S. Schumann, B. Gripaios and B. Webber, QCD jet rates with the inclusive generalized $k_{t}$ algorithms, JHEP 04 (2013) 089 [arXiv: 1212.5235] [INSPIRE].

[84] V. Del Duca, W. Kilgore, C. Oleari, C. Schmidt and D. Zeppenfeld, Higgs +2 jets via gluon fusion, Phys. Rev. Lett. 87 (2001) 122001 [hep-ph/0105129] [INSPIRE].

[85] V. Del Duca, W. Kilgore, C. Oleari, C. Schmidt and D. Zeppenfeld, Gluon fusion contributions to $H+2$ jet production, Nucl. Phys. B 616 (2001) 367 [hep-ph/0108030] [INSPIRE].

[86] F. Campanario and M. Kubocz, Higgs boson production in association with three jets via gluon fusion at the LHC: gluonic contributions, Phys. Rev. D 88 (2013) 054021 [arXiv:1306.1830] [INSPIRE].

[87] G. Cullen et al., Automated one-loop calculations with GoSam, Eur. Phys. J. C 72 (2012) 1889 [arXiv:1111.2034] [INSPIRE]. 
[88] G. Cullen et al., GoSam-2.0: a tool for automated one-loop calculations within the Standard Model and beyond, Eur. Phys. J. C 74 (2014) 3001 [arXiv:1404.7096] [InSPIRE].

[89] T. Gleisberg et al., Event generation with SHERPA 1.1, JHEP 02 (2009) 007 [arXiv:0811.4622] [INSPIRE].

[90] T. Binoth et al., A proposal for a standard interface between Monte Carlo tools and one-loop programs, Comput. Phys. Commun. 181 (2010) 1612 [arXiv:1001.1307] [INSPIRE].

[91] S. Alioli et al., Update of the Binoth Les Houches accord for a standard interface between Monte Carlo tools and one-loop programs, Comput. Phys. Commun. 185 (2014) 560 [arXiv: 1308.3462] [INSPIRE].

[92] P. Nogueira, Automatic Feynman graph generation, J. Comput. Phys. 105 (1993) 279 [INSPIRE].

[93] J.A.M. Vermaseren, New features of FORM, math-ph/0010025 [INSPIRE].

[94] J. Kuipers, T. Ueda, J.A.M. Vermaseren and J. Vollinga, FORM version 4.0, Comput. Phys. Commun. 184 (2013) 1453 [arXiv:1203.6543] [INSPIRE].

[95] G. Cullen, M. Koch-Janusz and T. Reiter, Spinney: a form library for helicity spinors, Comput. Phys. Commun. 182 (2011) 2368 [arXiv:1008.0803] [InSPIRE].

[96] T. Reiter, Optimising code generation with haggies, Comput. Phys. Commun. 181 (2010) 1301 [arXiv:0907.3714] [INSPIRE].

[97] G. Ossola, C.G. Papadopoulos and R. Pittau, Reducing full one-loop amplitudes to scalar integrals at the integrand level, Nucl. Phys. B 763 (2007) 147 [hep-ph/0609007] [INSPIRE].

[98] P. Mastrolia, G. Ossola, C.G. Papadopoulos and R. Pittau, Optimizing the reduction of one-loop amplitudes, JHEP 06 (2008) 030 [arXiv:0803.3964] [INSPIRE].

[99] G. Ossola, C.G. Papadopoulos and R. Pittau, On the rational terms of the one-loop amplitudes, JHEP 05 (2008) 004 [arXiv:0802.1876] [INSPIRE].

[100] P. Mastrolia, G. Ossola, T. Reiter and F. Tramontano, Scattering amplitudes from unitarity-based reduction algorithm at the integrand-level, JHEP 08 (2010) 080 [arXiv: 1006.0710] [INSPIRE].

[101] G. Heinrich, G. Ossola, T. Reiter and F. Tramontano, Tensorial reconstruction at the integrand level, JHEP 10 (2010) 105 [arXiv: 1008.2441] [INSPIRE].

[102] T. Binoth, J.-P. Guillet, G. Heinrich, E. Pilon and T. Reiter, Golem95: a numerical program to calculate one-loop tensor integrals with up to six external legs, Comput. Phys. Commun. 180 (2009) 2317 [arXiv:0810.0992] [INSPIRE].

[103] G. Cullen et al., Golem95C: a library for one-loop integrals with complex masses, Comput. Phys. Commun. 182 (2011) 2276 [arXiv:1101.5595] [InSPIRE].

[104] A. van Hameren, OneLOop: for the evaluation of one-loop scalar functions, Comput. Phys. Commun. 182 (2011) 2427 [arXiv:1007.4716] [INSPIRE].

[105] S. Catani and M.H. Seymour, A general algorithm for calculating jet cross-sections in NLO QCD, Nucl. Phys. B 485 (1997) 291 [Erratum ibid. B 510 (1998) 503] [hep-ph/9605323] [INSPIRE].

[106] T. Gleisberg and S. Höche, Comix, a new matrix element generator, JHEP 12 (2008) 039 [arXiv:0808.3674] [INSPIRE].

[107] S. Höche, Efficient dipole subtraction with Comix, in preparation. 
[108] T. Stelzer and W.F. Long, Automatic generation of tree level helicity amplitudes, Comput. Phys. Commun. 81 (1994) 357 [hep-ph/9401258] [INSPIRE].

[109] J. Alwall et al., MadGraph/MadEvent v4: the new web generation, JHEP 09 (2007) 028 [arXiv:0706.2334] [INSPIRE].

[110] R. Frederix, T. Gehrmann and N. Greiner, Automation of the dipole subtraction method in MadGraph/MadEvent, JHEP 09 (2008) 122 [arXiv:0808.2128] [INSPIRE].

[111] R. Frederix, T. Gehrmann and N. Greiner, Integrated dipoles with MadDipole in the MadGraph framework, JHEP 06 (2010) 086 [arXiv: 1004.2905] [INSPIRE].

[112] F. Maltoni and T. Stelzer, MadEvent: automatic event generation with MadGraph, JHEP 02 (2003) 027 [hep-ph/0208156] [INSPIRE].

[113] F. Wilczek, Decays of heavy vector mesons into Higgs particles, Phys. Rev. Lett. 39 (1977) 1304 [INSPIRE].

[114] K. Hamilton, P. Nason and G. Zanderighi, MINLO: multi-scale improved NLO, JHEP 10 (2012) 155 [arXiv: 1206.3572] [INSPIRE].

[115] Z. Bern et al., Ntuples for NLO events at hadron colliders, Comput. Phys. Commun. 185 (2014) 1443 [arXiv: 1310.7439] [INSPIRE].

[116] M. Cacciari and G.P. Salam, Dispelling the $N^{3}$ myth for the $k_{t}$ jet-finder, Phys. Lett. B 641 (2006) 57 [hep-ph/0512210] [INSPIRE].

[117] M. Cacciari, G.P. Salam and G. Soyez, The anti-kt jet clustering algorithm, JHEP 04 (2008) 063 [arXiv: 0802.1189] [INSPIRE].

[118] M. Cacciari, G.P. Salam and G. Soyez, FastJet user manual, Eur. Phys. J. C 72 (2012) 1896 [arXiv:1111.6097] [INSPIRE].

[119] H.-L. Lai et al., New parton distributions for collider physics, Phys. Rev. D 82 (2010) 074024 [arXiv: 1007.2241] [INSPIRE].

[120] C.F. Berger et al., Precise predictions for $W+3$ jet production at hadron colliders, Phys. Rev. Lett. 102 (2009) 222001 [arXiv:0902.2760] [INSPIRE].

[121] A.D. Martin, W.J. Stirling, R.S. Thorne and G. Watt, Parton distributions for the LHC, Eur. Phys. J. C 63 (2009) 189 [arXiv:0901.0002] [inSPIRE].

[122] R.D. Ball et al., Parton distributions with LHC data, Nucl. Phys. B 867 (2013) 244 [arXiv:1207.1303] [INSPIRE].

[123] J.D. Lykken, A.O. Martin and J.-C. Winter, Semileptonic decays of the Higgs boson at the Tevatron, JHEP 08 (2012) 062 [arXiv:1111.2881] [INSPIRE].

[124] C.F. Berger et al., Next-to-leading order QCD predictions for $W+3$-jet distributions at hadron colliders, Phys. Rev. D 80 (2009) 074036 [arXiv:0907.1984] [INSPIRE].

[125] M. Buschmann, D. Goncalves, S. Kuttimalai, M. Schönherr, F. Krauss and T. Plehn, Mass effects in the Higgs-gluon coupling: boosted vs off-shell production, JHEP 02 (2015) 038 [arXiv:1410.5806] [INSPIRE].

[126] J. Huston, private communication.

[127] M. Rubin, G.P. Salam and S. Sapeta, Giant QCD K-factors beyond NLO, JHEP 09 (2010) 084 [arXiv: 1006.2144] [INSPIRE].

[128] L.N. Lipatov, Reggeization of the vector meson and the vacuum singularity in non-Abelian gauge theories, Sov. J. Nucl. Phys. 23 (1976) 338 [Yad. Fiz. 23 (1976) 642] [InSPIRE]. 
[129] E.A. Kuraev, L.N. Lipatov and V.S. Fadin, The Pomeranchuk singularity in non-Abelian gauge theories, Sov. Phys. JETP 45 (1977) 199 [Zh. Eksp. Teor. Fiz. 72 (1977) 377] [INSPIRE].

[130] I.I. Balitsky and L.N. Lipatov, The Pomeranchuk singularity in quantum chromodynamics, Sov. J. Nucl. Phys. 28 (1978) 822 [Yad. Fiz. 28 (1978) 1597] [InSPIRE].

[131] C.R. Schmidt, A Monte Carlo solution to the BFKL equation, Phys. Rev. Lett. 78 (1997) 4531 [hep-ph/9612454] [INSPIRE].

[132] L.H. Orr and W.J. Stirling, Dijet production at hadron hadron colliders in the BFKL approach, Phys. Rev. D 56 (1997) 5875 [hep-ph/9706529] [INSPIRE].

[133] J.R. Andersen and A. Sabio Vera, Solving the BFKL equation in the next-to-leading approximation, Phys. Lett. B 567 (2003) 116 [hep-ph/0305236] [INSPIRE].

[134] J.R. Andersen and A. Sabio Vera, The gluon Green's function in the BFKL approach at next-to-leading logarithmic accuracy, Nucl. Phys. B 679 (2004) 345 [hep-ph/0309331] [INSPIRE].

[135] J.R. Andersen, On the role of NLL corrections and energy conservation in the high energy evolution of QCD, Phys. Lett. B 639 (2006) 290 [hep-ph/0602182] [INSPIRE].

[136] J.R. Andersen, The quark-antiquark contribution to the fully exclusive BFKL evolution at NLL accuracy, Phys. Rev. D 74 (2006) 114008 [hep-ph/0611011] [INSPIRE].

[137] J.R. Andersen and C.D. White, A new framework for multijet predictions and its application to Higgs boson production at the LHC, Phys. Rev. D 78 (2008) 051501 [arXiv:0802.2858] [INSPIRE].

[138] J.R. Andersen and J.M. Smillie, Constructing all-order corrections to multi-jet rates, JHEP 01 (2010) 039 [arXiv:0908.2786] [INSPIRE].

[139] J.R. Andersen, T. Hapola and J.M. Smillie, W plus multiple jets at the LHC with high energy jets, JHEP 09 (2012) 047 [arXiv:1206.6763] [INSPIRE].

[140] J.M. Campbell et al., Working group report: quantum chromodynamics, arXiv:1310.5189 [INSPIRE].

[141] T. Plehn, D.L. Rainwater and D. Zeppenfeld, Determining the structure of Higgs couplings at the LHC, Phys. Rev. Lett. 88 (2002) 051801 [hep-ph/0105325] [InSPIRE].

[142] T. Figy, V. Hankele and D. Zeppenfeld, Next-to-leading order QCD corrections to Higgs plus three jet production in vector-boson fusion, JHEP 02 (2008) 076 [arXiv:0710.5621] [INSPIRE].

[143] F. Campanario, T.M. Figy, S. Plätzer and M. Sjödahl, Electroweak Higgs boson plus three jet production at next-to-leading-order QCD, Phys. Rev. Lett. 111 (2013) 211802 [arXiv: 1308. 2932] [INSPIRE].

[144] S. Gangal and F.J. Tackmann, Next-to-leading-order uncertainties in Higgs + 2 jets from gluon fusion, Phys. Rev. D 87 (2013) 093008 [arXiv: 1302.5437] [INSPIRE].

[145] ATLAS collaboration, Measurements of Higgs boson production and couplings in the four-lepton channel in pp collisions at center-of-mass energies of 7 and 8 Te $V$ with the ATLAS detector, Phys. Rev. D 91 (2015) 012006 [arXiv:1408.5191] [INSPIRE].

[146] G. Klamke and D. Zeppenfeld, Higgs plus two jet production via gluon fusion as a signal at the CERN LHC, JHEP 04 (2007) 052 [hep-ph/0703202] [INSPIRE]. 